\title{
Pakistan: Third Review Under the Stand-By Arrangement
}

The following documents have been released and are included in this package:

- $\quad$ The staff report, prepared by a staff team of the IMF, following discussions that ended on November 12, 2009, with the officials of Pakistan on economic developments and policies. Based on information available at the time of these discussions, the staff report was completed on December 14, 2009. The views expressed in the staff report are those of the staff team and do not necessarily reflect the views of the Executive Board of the IMF.

- $\quad$ A staff statement.

- $\quad$ A Press Release.

- $\quad$ A statement by the Executive Director for Pakistan.

The document(s) listed below will be separately released.

Letter of Intent sent to the IMF by the authorities of Pakistan*

Memorandum of Economic and Financial Policies by the authorities of

Pakistan*

Technical Memorandum of Understanding*

*Also included in Staff Report

The policy of publication of staff reports and other documents allows for the deletion of market-sensitive information.

Copies of this report are available to the public from

International Monetary Fund • Publication Services

$70019^{\text {th }}$ Street, N.W. • Washington, D.C. 20431

Telephone: (202) 623-7430 • Telefax: (202) 623-7201

E-mail: publications@imf.org Internet: http://www.imf.org

\section{International Monetary Fund Washington, D.C.}




\title{
INTERNATIONAL MONETARY FUND
}

\section{PAKISTAN}

\section{Third Review under the Stand-By Arrangement}

\author{
Prepared by the Middle East and Central Asia Department \\ (In consultation with other departments)
}

Approved by Juan Carlos Di Tata and Michele Shannon

December 14, 2009

- Discussions were held during November 2-12, 2009 in Dubai. Staff met with Finance Minister Tarin, State Bank of Pakistan Governor Raza, Finance Secretary Siddique, and other senior officials. The IMF team consisted of Messrs. Mazarei (Head), Wieczorek, Kock, and Piazza (all MCD), Mr. Jonas (FAD), Mr. Sun (SPR), and Mr. Ross (resident representative in Pakistan). Ms. Kahkonen (World Bank) and Mr. Parvez (Asian Development Bank) participated in the discussions on structural issues. Mr. Ahmad (OED) also participated in the discussions.

- The SBA in the amount of SDR 5.169 billion (about \$7.6 billion, or 500 percent of quota) was approved by the Executive Board on November 24, 2008. The SBA was augmented to SDR 7,235.9 million ( $\$ 11,327$ million, or 700 percent of quota) and extended through the end of 2010 on August 7, 2009. The latest, third disbursement of SDR 766.7 million (\$1,200 million) was made on August 7, 2009, after completion of the second program review. Of this amount, SDR 475.6 million (\$745 million) was made available for budget financing. Disbursements under the program thus far amount to SDR 3,402.6 million ( $\$ 5,327$ million).

- In the attached Letter of Intent, the authorities request completion of the third review under the $\boldsymbol{S B} \boldsymbol{A}$. Completion of the review will enable the fourth disbursement under the program in the amount of SDR 766.7 million. Of this amount, SDR 237.75 million (about $\$ 380$ million) will be made available for budget financing.

- Program implementation has been uneven but key reforms are moving forward. All endSeptember quantitative performance criteria were met, with the exception of the ceiling on the overall budget deficit, which was missed by a margin of 0.3 percent of GDP due to a shortfall in external grants and nontax receipts from reimbursement of military outlays, lower tax revenue as the deadline for the advanced income tax payment was moved from September 15 to October 15 , and a spending overrun owing to higher military outlays and the advanced payment of October wages ahead of Ramadan. The tax revenue foregone in September was largely recovered in October, and the wage bill overrun was self-correcting. On these grounds, the authorities request a waiver of nonobservance for the related end-September 2009 performance criterion. All structural benchmarks relevant for the completion of the third review were met, albeit most of them with delays. Staff recommends the completion of the program review. 
Executive Summary

I. Background. .5

II. Recent Economic Developments and Outlook .5

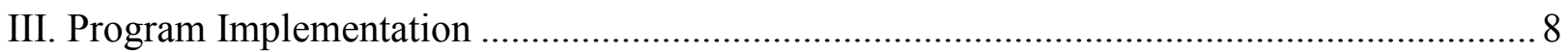

A. Fiscal Policy ................................................................................................... 8

B. Monetary Policy and Financial Sector Issues ......................................................... 9

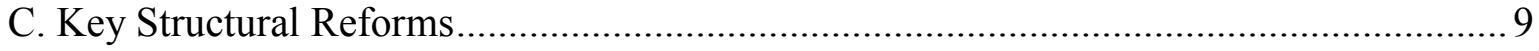

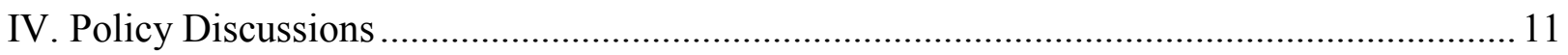

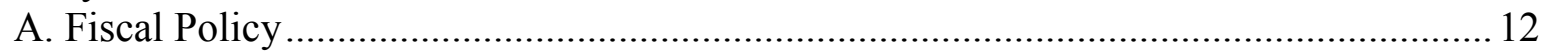

B. Structural Reforms in Tax Administration and Public Finance .................................. 13

C. Monetary and Exchange Rate Policy ..................................................................... 15

D. Financial Sector Issues .................................................................................. 16

V. Program Financing and Capacity to Repay the Fund .................................................. 16

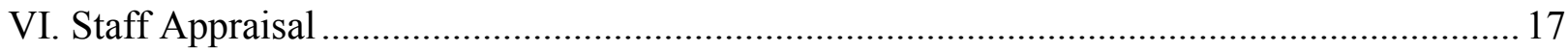

Box 1. Proposed Structural Benchmarks for the Second Half of 2009/10 ............................ 14

Figures

1. Selected Economic Indicators............................................................................... 19

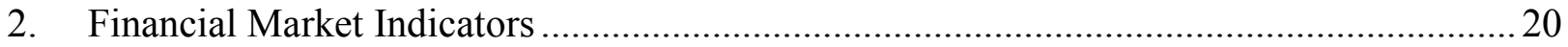

3. Real and External Sectors, 2005/06-2009/10 .......................................................... 21

4. Fiscal Policy Indicators, 2007/08-2009/10.......................................................... 22

Tables

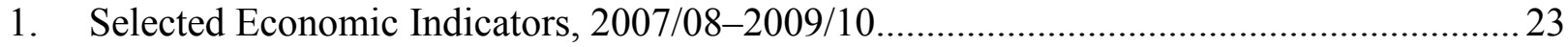

2. Balance of Payments, 2007/08-2009/10 .................................................................... 24

3a. Consolidated Government Budget, 2008/09-2009/10 (in billions of Pakistani rupees) ..... 25

3b. Consolidated Government Budget, 2008/09-2009/10 (in percent of GDP)..................... 26

4. Monetary Survey and Analytical Balance Sheet of the State Bank of Pakistan,

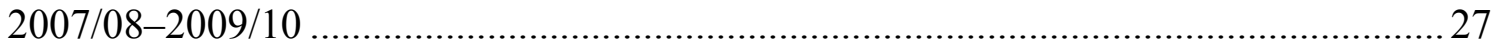

5. Medium-Term Macroeconomic Framework, 2007/08-2014/15 .................................... 28

6. Medium-Term Balance of Payments, 2007/08-2014/15 .............................................229

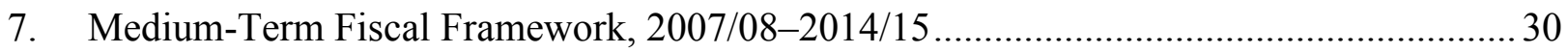

8. External Debt, 2007/08-2015/16 .................................................................... 31

9. Gross Balance of Payments Financing Requirements and Sources 2007/08-2009/10....... 32 
10. Indicators of Fund Credit, 2008/09-2015/16............................................................ 33

11. Access and Phasing under the Proposed Augmented Stand-By Arrangement ................... 34

12. Selected Vulnerability Indicators, 2007/08-2014/15 .............................................. 35

13. Financial Soundness Indicators for the Banking System............................................... 36

\section{Appendix}

Debt Sustainability Analysis

Attachments

I. Letter of Intent

II. Third Supplementary Memorandum on Economic and Financial Policies .....

III. Technical Memorandum of Understanding 


\section{EXECUTIVE SUMMARY}

Despite rising political and security uncertainties, economic stabilization is progressing. Economic activity remains weak, although there are signs of recovery in some sectors. Inflation has declined since mid-2009, but pressures have not abated. The external position has improved. Weak confidence has stymied credit to the private sector and nonperforming loans continue to grow. Banks, however, remain well-capitalized. All quantitative performance criteria for end-June and end-September were met, with the exception of the fiscal deficit targets.

Fiscal policy implementation remains challenging. In the first quarter of 2009/10, higher security spending, a shift in advanced income tax payments from September to October, and lower-than-expected external receipts and grants resulted in a fiscal slippage of 0.3 percent of GDP relative to the adjusted program target. Most of this slippage has been corrected in the second quarter of this fiscal year.

Looking ahead, security spending is expected to increase. Nonetheless, the authorities reaffirmed their intention to achieve the annual fiscal deficit target of 4.9 percent of GDP. The increase in security spending will be funded by adopting new revenue measures and cutting low-priority development spending. Timely disbursement of the pledged foreign financing is critical for meeting pressing social and development needs and avoiding excessive recourse to domestic financing.

Monetary policy has been generally cautious. The policy rate was lowered from 14 percent to 13 percent in August and to 12.5 percent in November. In the months ahead, the SBP will continue balancing the need to support the fragile recovery with its concerns about external stability and liquidity pressures arising from the government's large domestic financing needs.

The authorities have implemented most critical structural reforms, although some with significant lags. In particular, steps were taken to improve tax administration and introduce a VAT. The VAT is central for increasing revenue in order to reduce poverty and macrovulnerabilities and finance needed investment in human and physical capital. The target date of July 1, 2010 for the VAT introduction is ambitious, yet achievable, as underscored in the VAT action plan.

Despite the progress made on the stabilization front, Pakistan's underlying vulnerabilities remain high and have increased in some areas. In particular, vulnerabilities are fueled by continued revenue shortfalls, an increase in nonperforming loans, energy subsidies, and large dependence on commodity imports. A credible fiscal consolidation, strengthened competitiveness over the medium term, and improved governance are indispensable for reducing these vulnerabilities.

Staff recommends the completion of the third review under the Stand-By Arrangement. 


\section{BACKGROUND}

1. Political and security uncertainties have increased, raising risks to the program and the reform agenda. The political situation is fluid and economic reform does not command broad support, which limits the scope for implementing bold reform measures. The military campaign in South Waziristan that started in late October has resulted in significant security expenditure pressures and an outflow of the population from this area. Meanwhile, insurgents have staged a series of attacks in major cities. These political and security events continue to affect investor confidence.

\section{RECENT ECONOMIC DEVELOPMENTS AND OUTLOOK}

\section{Economic activity remains weak, although there are signs of recovery in some}

sectors. The wheat crop, which showed a 14 percent increase over the 2008 harvest, yielded a significant surplus (that could be exported), but the production of most other agricultural commodities dropped; notably, there was a 23 percent decline in sugar output. Despite low export demand and electricity shortages, the large-scale manufacturing production index showed a positive (year-on-year) growth in July-August 2009, owing mainly to a significant jump in cement production. The textile and automobile sectors are also showing some signs of revival. However, the continued slump in credit to the private sector suggests that investment remains low.

3. Inflation pressures have not yet abated. Headline and core inflation (y-o-y) dropped in October to 8.9 and 11 percent, respectively, in part due to the favorable base effect from peak inflation last year, but headline inflation rose to 10.5 percent in November while core inflation continued to decline and reached 10.6 percent. The rebound in inflation was due to recent food and petroleum product price increases and the electricity tariff hike in October. Although some of these pressures were temporary (Ramadan-related), the fiscal stance, and the price support policy (in the case of wheat), as well as electricity shortages and other supply-side factors have also contributed to inflation pressures.

4. The external position has continued to improve. The trade deficit in July-October 2009 narrowed relative to the same period of 2008, as lower imports more than offset the decline in exports. Workers' remittances continued to increase, and also contributed to a narrowing of the current account deficit. ${ }^{1}$ Official reserves, which had been under pressure in July, increased to $\$ 11.2$ billion at end-September, exceeding expectations, even after adjusting for an earlier-than-expected SDR allocation (about $\$ 1.3$ billion). Reserves declined subsequently to about $\$ 10$ billion in early December owing to substantial debt service

\footnotetext{
${ }^{1}$ The increase in remittances may be due in part to the authorities' efforts, under the Pakistani Remittances Initiative, to channel remittances through the banking system as well as an increase in the number of Pakistani migrant workers, particularly in Saudi Arabia, where demand for migrant labor services remained high.
} 
payments and a shortfall in external financing. SBP's intervention has been geared at achieving the program's reserve targets, but strong Hajj-related demand for foreign currency and inventory building for oil and other commodity imports have limited the scope for substantial foreign exchange purchases in recent weeks.

5. Disbursements of Tokyo pledges lag behind schedule, and the amount projected to be disbursed in 2009/10 has been revised downward. Disbursements of Tokyo-related assistance in 2009/10 have been delayed. The amount projected for 2009/10 has been reduced to $\$ 1.6$ billion ( $\$ 0.9$ billion lower than envisaged at the time of the second review), taking into account expectations that some donors will disburse their pledged support in the coming months. ${ }^{2}$

6. The rupee depreciated further by about 3 percent vis-à-vis the U.S. dollar during July-mid-December 2009. In real effective terms, the rupee appreciated by about 4.2 percent during September 2008-September 2009, reflecting the sizable inflation differential with trading partners. This has largely offset the competitiveness gains from the depreciation in 2008.

7. The lack of economic confidence has stymied credit and money growth. Broad money growth (y-o-y) has picked up since June. While the government's domestic financing needs have risen due to shortfalls in external financing, bank credit to the private sector has declined despite cuts in the policy interest rate. At the same time, the government's high borrowing requirements have pushed T-bill rates from just below $11 \frac{1 / 2}{2}$ percent in mid-July to $12 \frac{1}{4}$ percent in November. Government borrowing was supplemented by the issuance of government-guaranteed Term Finance Certificates (TFCs) to regularize the debt of the electricity sector companies (circular debt). Significant liquidity injections by the State Bank of Pakistan (SBP) in August-October were made to help meet the financing needs of the public sector and the seasonally higher demand for currency, while keeping the overnight repo rate within the policy corridor.

8. Domestic capital markets have been somewhat more upbeat. The Karachi Stock Exchange (KSE) index has increased by about 60 percent during January-November, despite a sharp drop in recent weeks triggered by security events and the developments in Dubai. The rebound in the KSE index mirrored the rally in other emerging stock markets and was supported by renewed foreign inflows (of about $\$ 300$ million since end-June). Following the completion of the second SBA review, Moody's improved Pakistan's B3 rating outlook to "stable" and confirmed it in early December, and Standard \& Poor's upgraded Pakistan's rating to "B-minus" in late September on account of increased IMF disbursements and

\footnotetext{
${ }^{2}$ The availability and timing of disbursements beyond 2009/10 (originally estimated at over $\$ 3$ billion) remain uncertain. The U.S. Kerry-Lugar Bill, which authorizes aid of $\$ 1.5$ billion a year during the U.S. fiscal years 2010-14, was passed in late September.
} 
improved political stability. Pakistan's EMBIG spread narrowed from 1,037 bps in June to 606 bps in early-December.

9. Overall, economic confidence remains subdued. The stock market performance has not been matched by a revival of long-term interest in Pakistan's economy, as witnessed by the low level of FDI during July-September (\$463 million). Continued concerns over the domestic security situation, power shortages, lack of progress in addressing governance issues, and difficulties with contract enforcement feature prominently among the factors identified as hampering FDI. ${ }^{3}$

\section{Banks remain well-capitalized, but nonperforming loans have continued to grow.}

Financial soundness indicators at end-September showed a further increase in nonperforming loans (NPLs) and some decline in bank profitability due to increased provisioning. At the same time, the risk-weighted capital adequacy ratio (CAR) indicates that the banking system is solvent even if all outstanding NPLs were to become irrecoverable. This conclusion is supported by standard stress tests performed by the SBP on end-June data. ${ }^{4}$ The improvement in the risk-weighted CAR reflected mainly a further shift in banks' assets toward government and government-guaranteed instruments.

\section{The 2009/10 outlook for growth remains unchanged, with inflation rising and the current account improving.}

- $\quad$ The real GDP growth projection of 3 percent for 2009/10 remains unchanged, although downside risks are high.

- The inflation outlook (y-o-y) has been revised from 9 to 11 percent. This reflects the rebound in the prices of fuel and a larger second round impact of the increases in electricity tariffs.

- $\quad$ Higher remittances and other private transfers have resulted in an improved current account outlook, notwithstanding higher oil and other commodity prices. The current account deficit is now projected at 4.2 percent of GDP, compared with 4.7 percent at the time of the second review. However, the impact on the overall balance of payments will likely be muted by lower Tokyo-related disbursements.

\footnotetext{
${ }^{3}$ Pakistan is ranked $85^{\text {th }}$ among 183 countries covered by the latest Doing Business report and $139^{\text {th }}$ out of 180 countries covered by the latest Corruption Perception Index. The anti-corruption agenda initiated in July 2007 remains largely unfinished. Key concerns listed by Transparency International include: (i) procurement, which costed the development budget an estimated 11/2 percent of GDP in 2007/08; and (ii) monopolistic practices. According to the findings reported by the Auditor General Office, corruption costs the Pakistan's Treasury 3.5-4.0 percent of GDP a year in tax revenue and results in a misuse of 20-30 percent of development expenditure.

${ }^{4}$ For the latest SBP review of the banking system, see: http://www.sbp.gov.pk/publications/q_reviews/qpr.htm.
} 


\section{Program Implementation}

12. The quantitative performance criteria for end-June and end-September were met, with the exception of the fiscal deficit target. ${ }^{5}$ Despite measures taken to strengthen budget execution since end-June, the fiscal deficit target for end-September was missed by about 0.3 percent of GDP. (The headline deficit target was missed by about 0.2 percent of GDP, but the adjustment of the target on account of the shortfall in external grants increased the overrun to 0.3 percent of GDP.) All other end-September quantitative performance criteria were met with comfortable margins. Most notably, the end-September adjusted program floor for the net foreign assets of the SBP was exceeded by about $\$ 1.3$ billion (see Supplementary Memorandum of Economic and Financial Policies (SMEFP), Table 1).

\section{A. Fiscal Policy}

13. Fiscal management remains difficult. In the first quarter of 2009/10, a combination of weak economic activity, a shift in the advanced income tax payments from September to October, lower grants, and lower-than-expected receipts from the Coalition Support Fund for reimbursement of certain military outlays resulted in a revenue shortfall of about 0.6 percent of GDP relative to the program target (roughly evenly split among tax revenue, nontax revenue, and grants). ${ }^{6}$ On the expenditure side, higher security spending, and the early payment of wages for October ahead of Eid contributed 0.2 percent of GDP to the slippage.

\section{The authorities took several measures to contain the first quarter fiscal slippage.}

These measures included compression of spending on goods and services and investment and the mobilization of a transfer from the SBP equivalent to about $1 / 2$ percent of GDP.

\section{Spending in high-priority areas, such as the social safety net and assistance for}

IDPs, has been lower than expected. The rollout of the strengthened targeting scheme under the Benazir Income Support Program (BISP) continued to be challenged by the implementation capacity. As a result, spending under the BISP amounted only to Rs. 10.4 billion in the first quarter, compared with Rs. 14 billion projected under the program. However, some additional social spending, mainly through subsidization of basic foodstuffs, was incurred by the provinces. Spending on IDPs was about two-thirds of the Rs. 15.5 billion (about 0.1 percent of GDP) budgeted for the first quarter of 2009/10, as the security situation in Swat stabilized faster than expected.

\footnotetext{
${ }^{5}$ In August, the Executive Board approved a waiver of applicability of the quantitative performance criteria for end-June. Up-to-date data indicate that the end-June fiscal deficit target was missed by Rs. 118 billion (about 0.9 percent of GDP) rather than Rs. 121 billion, as reported in the staff report for the second review (Country Report Number 09/265).

${ }^{6}$ Compared with the previous year, tax and nontax revenue collection grew by about 11 percent, approximately in line with year-on-year inflation, with tax revenue increasing by about 7 percent.
} 


\section{B. Monetary Policy and Financial Sector Issues}

16. In general, monetary policy has been cautious. The SBP discount rate was lowered from 14 percent to 13 percent in August, and to 12.5 percent in November, but real interest rates remained positive. The rate cuts were limited because of concerns about remaining inflation pressures, the fiscal slippage in the first quarter, and the availability of external financing.

17. The introduction of the new liquidity management framework went smoothly. In mid-August, the SBP introduced an explicit interest rate corridor of 300 bps (between the discount rate and the rate on the newly established overnight deposit facility). Consistent with the new framework, the SBP shortened the periodicity of monetary policy decisions from quarterly to bimonthly in order to facilitate more frequent adjustments of policy rates. Following the introduction of the corridor, fluctuations in the overnight repo rate have fallen significantly.

18. The SBP has also increased interest rates on its refinancing facilities, with a view to aligning them with market rates over the next two years. To this end, the interest rates on the Export Finance Scheme and the Long Term Financing Facility have been increased to 8 percent and 9.2-10.25 percent (depending on tenor), respectively. ${ }^{7}$

19. The SBP has introduced temporary changes in provisioning rules to promote the restructuring of NPLs by creditors. The new rules, which will expire automatically on June 30, 2010, include: (i) expanding the base of eligible collateral and allowing a higher forced sale value; and (ii) reducing provisioning requirements for restructured NPLs. Although the new rules introduce some degree of regulatory forbearance, the SBP has put in place measures to curb the moral hazard risks associated with possible "ever-greening" of NPLs, including by requiring monthly reporting on the rescheduled NPLs and making the benefits derived from relaxed provisioning ineligible for dividend payment and usable solely for strengthening bank reserves.

\section{Key Structural Reforms}

20. The authorities implemented most structural reforms in the areas critical for the program, although some of them with significant lags.

- $\quad$ The legislative amendments to enhance the effectiveness of the SBP's enforcement powers in banking supervision were sent to parliament in early October (structural benchmark for end-August) and amendments to the SBP law to strengthen the central bank's operational independence will be submitted to parliament by December 20 .

\footnotetext{
${ }^{7}$ Continued recourse to these preferential refinancing windows at the SBP is estimated to result in quasi-fiscal costs amounting to $0.1-0.2$ percent of GDP a year.
} 
Meanwhile, the SBP has appointed a nine-member Monetary Policy Committee whose role and legal status are included in the proposed amendments to the SBP Act.

- $\quad$ The key steps in tax administration reform have been completed (structural benchmark for end-September). On September 12, the government approved, via an ordinance, regulations to form a new occupational group (Inland Revenue Service) in the Federal Bureau of Revenue (FBR). On October 28, a presidential ordinance was issued to harmonize the income tax and the general sales tax laws, in line with the new administrative structure. On November 11, the FBR issued notifications to revise the structures of Large Taxpayer Units (LTU) and Regional Taxpayer Offices (RTO), notwithstanding legal challenges filed by disgruntled customs officers. The Ministry of Finance has also instructed the FBR to start tax audits and has provided the necessary funds for external auditors.

- $\quad$ Limits on provincial borrowing have been put in place. Ceilings on the overdraft to provinces from the SBP (equal to six weeks of the respective provincial wage bills) were established in early October. At the same time, the provincial overdrafts outstanding at end-June have been converted into loans to be amortized over four years.

- $\quad$ Considerable work has been completed for the introduction of the VAT by July 1, 2010. A September 2009 conference marked the launch of the process of consultation on the VAT. Subsequently, a drafting workshop was held in Dubai in early November to clarify the scope of legal preparations, which yielded a plan for presenting the VAT law to parliament by end-December (structural benchmark). Preliminary understandings have been reached within the government that VAT revenues attributable to services would be distributed to the provinces before the VAT monies go to the divisible pool. This agreement is essential for provincial buy-in for a consolidated administration of a broad-based VAT — crucial both for higher revenue and effective tax administration. Finally, draft legislation is being prepared by a government team in consultation with international experts.

- $\quad$ Electricity tariffs were raised on average on October 1 by $4 \frac{1}{4}$ percent rather than by 6 percent as agreed with the World Bank and the Asian Development Bank (ADB), due to differential application of the adjustment. The remaining $13 / 4$ percent will be implemented together with the regular quarterly adjustment due by December 20 . The additional costs accrued as a result of the delayed monthly adjustments (which were to be in effect since end-July) will also be covered through the quarterly tariff adjustment, together with other costs accrued in the first quarter of 2009/10.

Electricity tariffs are being adjusted on a monthly basis on account of increases in fuel and power purchase prices; at end-October they were adjusted to reflect price changes in August and September, and thereafter they have been adjusted on a monthly basis. The authorities have indicated that electricity tariffs will be increased 
by an additional 12 percent on January 1, 2010, in line with agreements reached with the World Bank and the ADB. ${ }^{8}$

- In mid-September, the government guaranteed Rs 85 billion ( 0.6 percent of GDP) in TFCs to settle a portion of the energy sector's "circular debt." The placement was more difficult than expected, as some banks refused to participate on the grounds that their exposure to the energy sector was already excessive. ${ }^{9}$ The remainder of the circular debt, which amounted to Rs 216 billion (1.3 percent of GDP) as of July 1, 2009 , will be transferred to a holding company under the Ministry of Water and Power by end-March 2010. The exact amount of these liabilities may change once an audit - currently underway - is completed.

- $\quad$ The rollout of the strengthened targeting system for the BISP has been delayed. The program of Rs. 70 billion ( 0.5 percent of GDP) was intended to provide support for five million families in 2009/10 and to be extended to seven million families by 2010/11. However, only part of BISP resources budgeted for 2009/10 is expected to be delivered using the new enhanced targeting system. A pilot using the enhanced system to identify poor families has been completed in 15 districts. The World Bank and the authorities are discussing ways to expedite the BISP rollout, including completion of the nationwide survey. Meanwhile, to accelerate disbursements under the BISP and help poor families, the government has launched the Wasilla-ul-Haq credit program under which interest-free loans of Rs. 300,000 $(\$ 3,600)$ will be given to selected BISP beneficiaries to start their own businesses.

\section{Policy Discussions}

\section{Discussions focused on:}

- $\quad$ Ensuring that fiscal policy achieves the deficit target of 4.9 percent of GDP (excluding grants), while providing for additional security expenditures. ${ }^{10}$

- $\quad$ Maintaining the momentum of structural reform (including structural benchmarks for the second half of 2009/10), notably in the areas of tax policy, tax administration, and public financial management.

\footnotetext{
${ }^{8}$ See Box 1 in the staff report for the second review under the SBA (IMF country report No. 09/265) for further details about the electricity sector.

${ }^{9}$ This round of TFCs was issued at the Karachi Inter-Bank Offered Rate (KIBOR) plus 200 bps, while a similar placement of Rs. 80 billion in the spring of 2009 was made at KIBOR plus 175 bps.

${ }^{10}$ The 2009/10 budget deficit target (excluding grants) is 4.6 percent of GDP. Based on the assumption of additional budgetary grants for IDPs, the authorities increased the fiscal deficit target (excluding grants) to 4.9 percent of GDP, as reflected in the budget approved by parliament. The authorities intend to limit the deficit by any shortfall in IDP grants (up to 0.3 percent of GDP), although they will ensure that spending on IDPs is protected and make offsetting expenditure cuts elsewhere.
} 
- $\quad$ Monetary and exchange rate policies.

- $\quad$ Financial sector soundness, factors explaining the private credit slump, and the implications of higher government borrowing from domestic sources.

- $\quad$ Establishment of performance criteria for end-March and end-June.

\section{A. Fiscal Policy}

\section{The first quarter 2009/10 slippage has been largely reversed, but some additional} fiscal space will be required in the second quarter because of increased security spending. As noted earlier, the overrun related to the advance wage payment was self-reversing, and the strong growth in income tax collections in October suggests that the first quarter tax revenue shortfall has also been largely eliminated. On this basis, the authorities request a waiver of nonobservance for the end-September performance criterion on the overall budget deficit. In addition, to avoid undue cuts in other priority spending while accommodating urgent security expenditures, the authorities request, and staff supports, the end-December fiscal deficit target to be relaxed by 0.1 percentage of GDP, irrespective of a possible shortfall in IDP grants.

\section{Nonetheless, the authorities reaffirmed their intention to achieve the annual fiscal} deficit target of 4.9 percent of GDP. To contain the annual deficit at this level and make room for higher security outlays, the authorities will have to reprioritize spending - including through cuts in development expenditure — and boost revenues. ${ }^{11}$ The authorities have already identified a possible reduction of exemptions for the capital value tax and contingency expenditure measures (lower-priority development expenditure and subsidies) that can be deployed to cover revenue or external financing shortfalls, or higher than anticipated security outlays. The deterioration in security may also result in renewed spending pressures for assistance to IDPs. The authorities believe, however, that IDP needs could be met from the existing IDP budgetary allocation. ${ }^{12}$ They also recognized that if Tokyo-related disbursements are substantially lower than projected, the deficit would need to be reduced further, possibly by up to 0.4 percent of GDP. ${ }^{13}$

\footnotetext{
${ }^{11}$ Additional security spending (over and above the budgeted amounts) is currently estimated at Rs. 65 billion ( 0.4 percent of GDP); however, the total amount of additional security spending would depend on the course of campaign and could be higher.

${ }^{12}$ Approximately 170,000 people have fled South Waziristan.

${ }^{13}$ Under the program framework, up to $\$ 1.4$ billion ( 0.8 percent of GDP) in shortfalls of the projected $\$ 2.2$ billion in Tokyo-related disbursements from bilateral donors would continue to be covered by Fund resources at year's end. In this event, the budget will replenish the dedicated account as soon as the delayed disbursements are received in the following year. Fund resources would not be used to cover shortfalls in the disbursement of pledges from multilateral donors.
} 
24. The authorities will adopt new revenue measures. They have decided to increase the broad-based special excise duty rate from 1 to 5 percent, which could yield about Rs. 28 billion in additional revenues in the second half of FY 2009/10 (about 0.4 percent of GDP on an annualized basis). Furthermore, a number of measures to improve tax administration are being taken, including tax audits, automated refunds, and measures to reduce the number of under-reporting taxpayers and nonfilers (SMEFP, $₫ 13$ ).

25. The fiscal outlook for FY 2009/10 is subject to several risks. These risks include: (i) a worsened security situation, which could hurt confidence and economic activity with adverse implications for revenue; and (ii) simultaneous tax administration reforms and the introduction of the VAT creating a significant workload for tax officials that could temporarily weaken tax collections. Also, (iii) failure to implement the agreed adjustment in electricity tariffs would increase pressure for power subsidies, (iv) spending compression may be difficult to sustain, especially if security-related spending pressures increase; and (v) external financing may be lower than projected.

26. In this context, timely disbursement of the pledged foreign financing is critical. On current projections, the IMF budget financing would extend beyond end-June 2010 to cover a shortfall in Tokyo-related disbursements from bilateral donors of about $\$ 700$ million. The authorities are concerned that a further shortfall in the disbursement of Tokyo pledges will result in a tightening of the fiscal deficit target, which may lead to an undue compression of social and development spending.

\section{B. Structural Reforms in Tax Administration and Public Finance}

27. The authorities identified several key measures for the remainder of 2009/10 aimed at implementing the VAT and strengthening public financial management (Box 1).

- $\quad$ Progress toward the full implementation of the VAT by July 1, 2010 (proposed structural benchmark) will be tracked based on a time-bound action plan. The harmonization of the VAT with other tax laws and the introduction of the automated tax refund system in all LTOs and RTUs by end-March 2010 (proposed structural benchmark) will be key steps. Passage of the VAT law by April 2009 will be a key milestone.

- $\quad$ To strengthen expenditure control and improve governance, the Ministry of Finance will: (i) conduct a survey of the federal government entities accounting jointly for 90 percent of the remaining cash balances in commercial banks in order to ensure a proper use of assignment accounts under the treasury single account (TSA); (ii) transfer the remaining balances to the TSA and close all the commercial bank accounts of those agencies by end-June 2010; and (iii) complete the transition of all provincial government accounts to the assignment account mechanism by end-June 2010 ((ii) and (iii) — proposed structural benchmark). 
- $\quad$ To improve budget preparation, execution, and reporting, the Ministry of Finance will: (i) strengthen the medium-term budget framework to ensure that the recurrent costs of development programs are properly reflected in the annual budgets (SMEFP, ๆ16); and (ii) launch an independent review-to be completed by end-June 2010 (proposed structural benchmark) — of the Project for Improving Financial Reporting and Auditing (PIFRA).

- $\quad$ The authorities remain committed to putting the electricity sector on a sound financial footing and will adhere to the timetable (agreed with the World Bank and the ADB) for electricity tariff increases and the automatic system of monthly and quarterly tariff adjustments to eliminate tariff differential subsidies by August 2010. To this effect, the ordinance amending the National Electric Power Regulatory Authority (NEPRA) Act issued in July that provides for the monthly surcharge and quarterly determination of overall electricity tariffs has been renewed to ensure that the amendments under the NEPRA Act remain in force.

- $\quad$ As the full rollout of the BISP will require additional capacity building, a set of measures will be taken to accelerate the implementation of the social safety net (SMEFP, ๆ15).

\section{Box 1. Proposed Structural Benchmarks for the Second Half of 2009/10}

The following measures are deemed macro-critical and proposed to be structural benchmarks:

- $\quad$ Implement an expedited electronic sales tax refund system in all RTOs and LTUs by endMarch, 2010. This measure is critical for a successful introduction of the VAT. It will ensure direct input of refund requests and prompt processing and confirmation of refunds.

- $\quad$ Complete an independent review of PIFRA by end-June 2010 to improve fiscal reporting, lay the basis for commitment control, and assess in which institution PIFRA will be located.

- $\quad$ Move to a Single Treasury account by completing the transition of deposits of the largest federal spending agencies to a single treasury account and the transition of provincial government accounts to the assignment account mechanism by end-June 2010, in order to improve expenditure control and cash management.

- $\quad$ Introduce the VAT by July 1, 2010, to broaden the revenue base and improve medium-term fiscal sustainability. 


\section{Monetary and Exchange Rate Policy}

\section{Remaining inflation pressures and increased domestic financing of the public} sector prevent the SBP from easing monetary policy to support growth. Concerns about low economic activity, weak private credit demand, and lower y-o-y headline inflation point in the direction of an easing of the monetary stance. However, the risks of inflationary pressures due to higher oil prices and electricity tariff adjustments and the high domestic financing needs of the government in the forthcoming months were recognized by the authorities. The SBP proceeded with a reduction of the policy interest rate of $50 \mathrm{bps}$ in November. Staff would have given greater weight to inflation risks and preferred a more cautious stance. In the months ahead, the SBP will continue to balance its desire for a more forceful support of the fragile recovery with its concerns about external stability and liquidity pressures arising from the government's large domestic financing needs.

\section{The SBP will strengthen its liquidity management operations to ensure that the} overnight repo rate remains close to the center of the interest rate corridor (150 bps below the policy rate). It will also continue to manage liquidity injections (or mop-ups) in order to deal solely with temporary liquidity fluctuations and stabilize short-term interest rates. Persistent liquidity pressures will be addressed by adjusting the overall monetary policy stance. Staff stressed that interest rate and exchange rate policies would need to be flexible in light of the forthcoming financing pressures and the recent drop in international reserves.

30. The SBP intends to continue to capitalize on the improved external current account outlook to further strengthen reserves and has decided to complete the phasing out of direct foreign exchange provision for oil imports ahead of schedule.

- Given the improved current account outlook, the SBP will continue to build reserves. Consequently, the net foreign assets targets under the program have been raised. ${ }^{14}$ The SBP will also maintain a flexible exchange rate policy to ensure that these targets are met. Greater exchange rate flexibility would also enhance the effectiveness of the interest rate corridor and reduce the SBP's heavy reliance on open market operations to manage liquidity.

- $\quad$ The SBP announced in early December that it will cease provision of foreign exchange for crude oil imports by December 14, 2009. This will complete the phasing out of the SBP foreign exchange provision for imports of oil and petroleum products and deepen the foreign exchange market.

\footnotetext{
${ }^{14}$ The end-December target for the SBP's net domestic assets has also been modified.
} 


\section{Financial Sector Issues}

\section{Discussions on financial sector issues focused on the decline in private sector credit, the increase in NPLs, and actions to help several small banks meet the enhanced minimum capital requirements.}

- Increased risk aversion among banks is a key factor behind the shift in lending away from the private sector to the government and government-guaranteed paper. The decline in private sector credit has also reflected lower demand for working capital. Moreover, since mid-2008/09 a significant part of domestic credit has been channeled to government-guaranteed commodity operations (mainly related to wheat procurement). The SBP remains concerned that continued risk aversion by banks may further limit lending to enterprises and hinder the economic recovery.

- $\quad$ Although the SBP thinks that the growth of NPLs does not threaten banking system stability, it also believes that banks have not been sufficiently proactive in seeking the repayment or restructuring of NPLs, which has compounded the credit slump. To help overcome this inertia, the SBP recently introduced temporary changes to the provisioning rules with respect to the rescheduled NPLs which aim to promote bilateral workouts and contribute to a resumption of credit. ${ }^{15}$ The SBP agreed with the staff that intensified on- and off-site supervision should seek to avoid moral hazard from this temporary change in provisioning rules. The proposed bankruptcy law, when passed, will also facilitate the repayment or restructuring of NPLs.

- $\quad$ Banks are generally well-capitalized, but several small banks will need to merge or receive capital injections to be able to meet the new minimum capital requirements (increased from Rs. 5 billion at present to Rs. 6 billion at end-2009). Plans to this effect have been agreed upon between bank owners/shareholders and the SBP. ${ }^{16}$

\section{Program Financing and Capacity to Repay the Fund}

\section{External financing remains adequate despite the projected shortfall in Tokyo-}

related disbursements. Higher remittances and other transfers are expected to more than compensate for the shortfall, and gross reserves are envisaged to strengthen further, notwithstanding some increase in political and security uncertainty. The assessment of Pakistan's capacity to repay the Fund remains broadly unchanged, but is subject to higher downside risks. While higher reserves provide a cushion, uncertainties are also higher, including those linked to donor financing and commodity price trends.

\footnotetext{
${ }^{15}$ The SBP clarified that the changes to the provisioning rules and rescheduling of NPLs will not result in a reclassification of NPLs.

${ }^{16}$ Fitch recently withdrew the ratings of four major Pakistani banks, pointing to lack of investor interest and significant asset quality challenges that these banks may face over the medium term. The authorities clarified that this decision was unlikely to have any material effect as the Fitch rating was not solicited by these banks, whose activity is largely oriented to the domestic market.
} 


\section{StafF ApPraisal}

33. Program implementation has been uneven but most key reforms are moving forward. The delays in the implementation of structural reforms were partly the result of institutional inertia and political pressures. Nevertheless, considering the difficult security circumstances and the continued unfavorable domestic and external economic conditions, the authorities have made significant efforts to stay the course on stabilization and structural reform.

34. Despite the strengthened external position and better market sentiment, Pakistan's underlying vulnerabilities remain high and have increased in some areas. In particular, vulnerabilities relate to continued revenue shortfalls, weak credit performance, energy subsidies, and large dependence on commodity imports. A credible fiscal consolidation, together with flexible interest rate and exchange rate policies and improved governance are indispensable for reducing these vulnerabilities and improving economic performance.

\section{The fiscal slippage in the first quarter of 2009/10 points to the need for better} planning and use of contingencies. Some of the factors which led to the fiscal overrun in the first quarter were known in advance, and the slippage may have been prevented. Looking forward, the authorities are encouraged to avoid further fiscal slippages to build confidence in the economic policies. The identified contingencies should make this feasible and reduce risk to the fiscal program. New slippages would also heighten the risk to capacity to repay the Fund.

36. The authorities' resolve to maintain the 2009/10 fiscal target will help preserve macroeconomic stability and limit the potential for crowding out of the private sector. Against the backdrop of inflationary pressures and declining private sector credit, this strengthened focus on fiscal discipline is particularly critical. Timely disbursement of the pledged foreign financing will facilitate fiscal management and avoid excessive recourse to domestic borrowing.

37. The introduction of the VAT and associated administrative reforms must proceed as planned. The VAT is central for strengthening revenue in order to reduce poverty and macro-vulnerabilities and finance needed investment in human and physical capital. The target date of July 1, 2010 for the VAT introduction is ambitious, but achievable. Passage of the VAT law by end-April 2010 is a key milestone. Meeting the benchmarks for the submission of the VAT law to parliament and introducing the automated tax refund system will play pivotal roles in this respect.

38. It is important to address the delay in the nationwide rollout of the strengthened targeting system for the social safety net. Strengthening the social safety net is at the heart of the government's poverty reduction efforts and is crucial to maintain social stability and ease the hardship related to macroeconomic adjustment. 
39. Monetary policy should continue to be cautious and accompanied by exchange rate flexibility. The SBP should focus on counteracting ongoing inflation pressures from sources beyond its control. In this context, its primary focus should remain to strengthen its foreign reserves position and foster bank soundness. In addition, the SBP should remain vigilant that bank lending decisions are economically sound and be alert to moral hazard risks related to regulatory forbearance.

40. The interest rate corridor has been working smoothly but could be better integrated in the monetary policy objectives. The SBP should take advantage of its increased independence and clarify its policy objectives, which will help ensure that interest rate policy is properly interpreted by the market. Most notably, greater exchange rate flexibility would help the effectiveness of the interest rate corridor.

41. Despite the challenges faced, the authorities have made significant efforts to keep the program on track. The overall policy stance has helped bring inflation down from the high levels last year, strengthened the external position and furthered macroeconomic stabilization against a background of adverse security developments, weak external demand, and a rapidly changing political environment. Some of the factors behind the nonobservance of the end-September fiscal deficit performance criterion were temporary and the authorities have identified measures to augment revenue and control expenditure. The progress made in tax, electricity and financial sector reforms attests to the authorities' determination to press ahead with reforms in these key areas. In this light and based on the strength of the updated commitments expressed by the authorities in the attached SMEFP, staff supports the requested waiver of nonobservance for the end-September quantitative performance criterion on the fiscal deficit, and the modifications of the end-December performance criteria on the fiscal deficit and on the net foreign assets and net domestic assets of the SBP, and recommends the completion of the third review. 
Figure 1. Pakistan: Selected Economic Indicators (November 2008-November 2009, unless otherwise indicated)

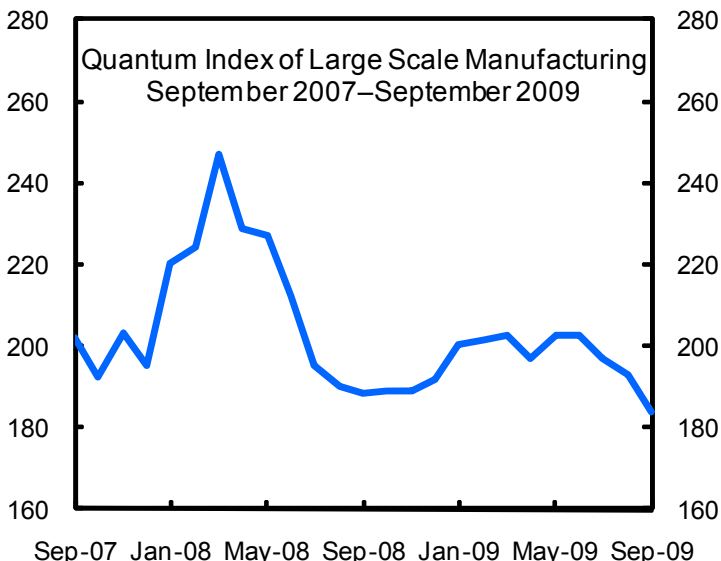

Sep-07 Jan-08 May-08 Sep-08 Jan-09 May-09 Sep-09

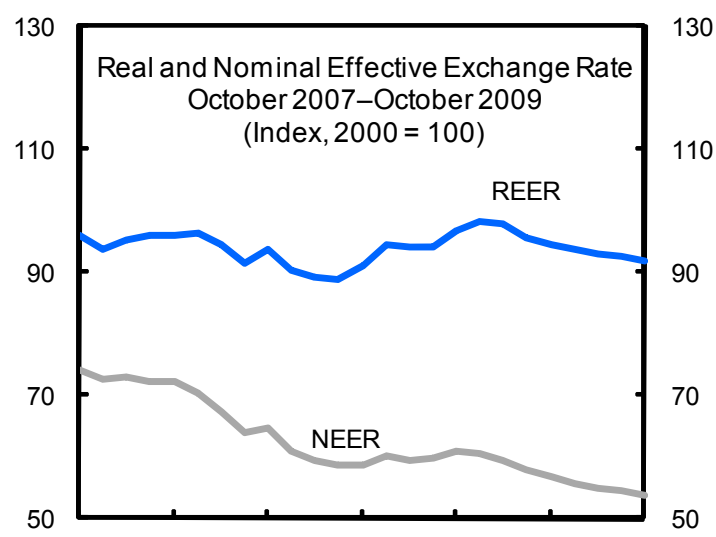

Oct-07 Feb-08 Jun-08 Oct-08 Feb-09 Jun-09 Oct-09

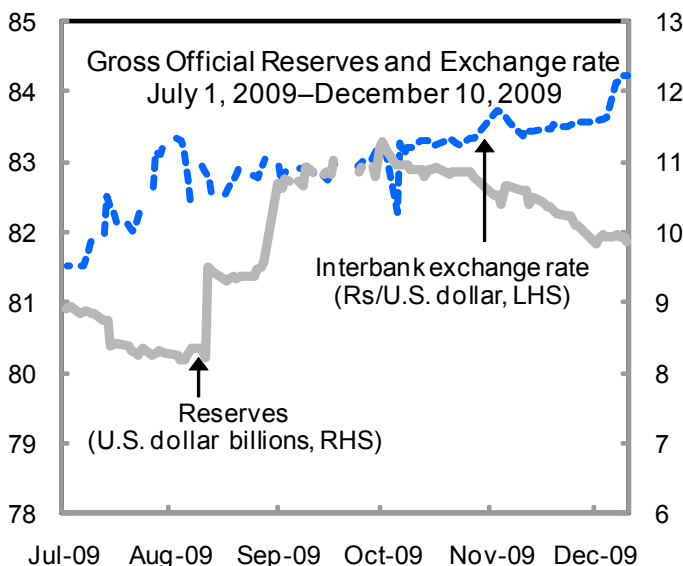

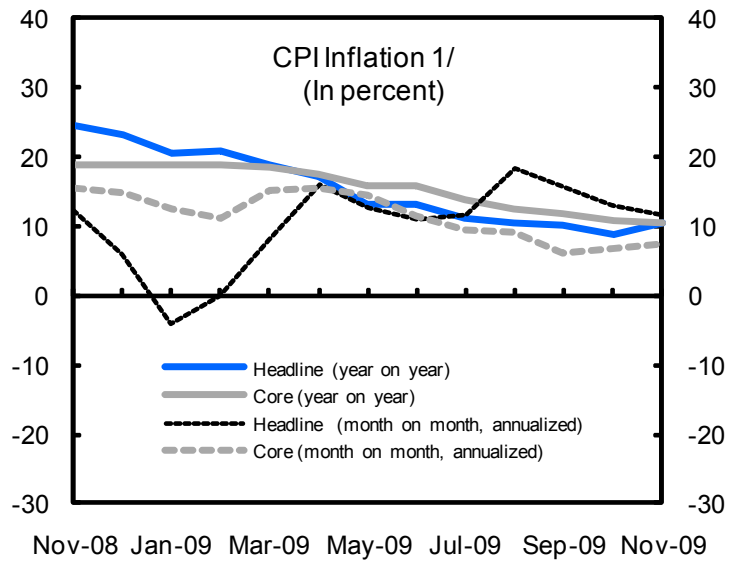

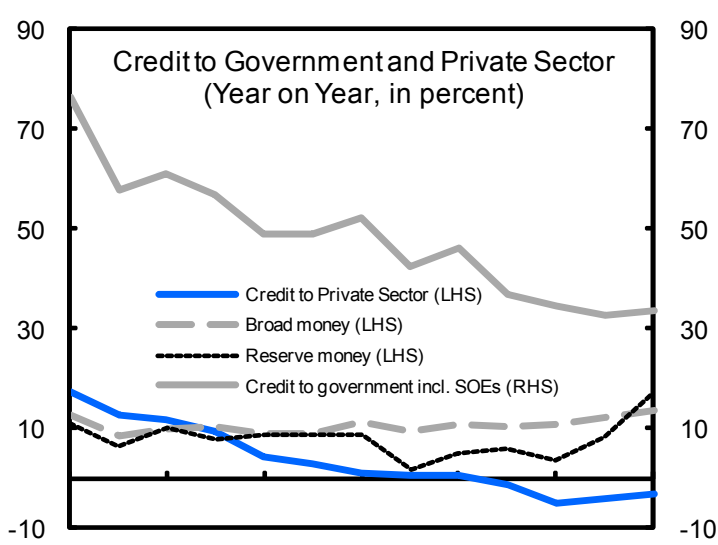

Nov-08 Jan-09 Mar-09 May-09 Jul-09 Sep-09 Nov-09

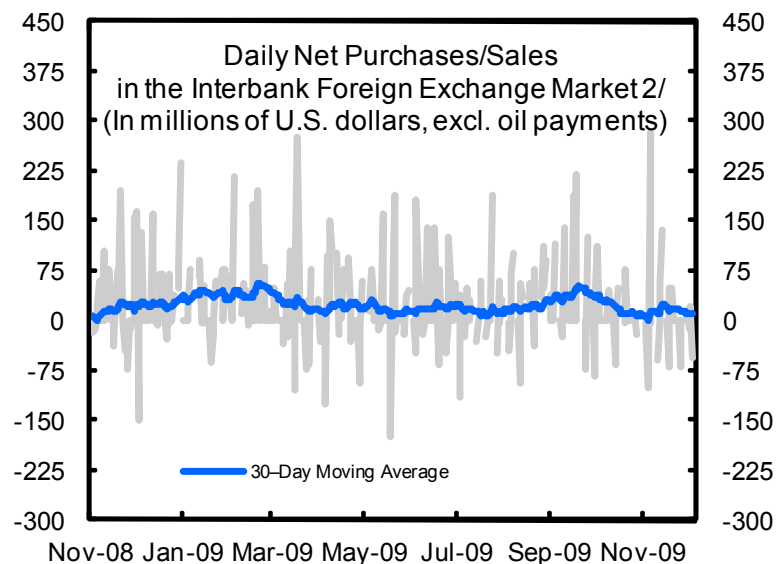

Source: Pakistani authorites.

$1 /$ Annualized month on month core and headline inflation are based on a three month moving averages of the respective indices.

2/Positive values indicate net purchases in the interbank market. 
Figure 3. Pakistan: Real and External Sectors, 2005/06-2009/10
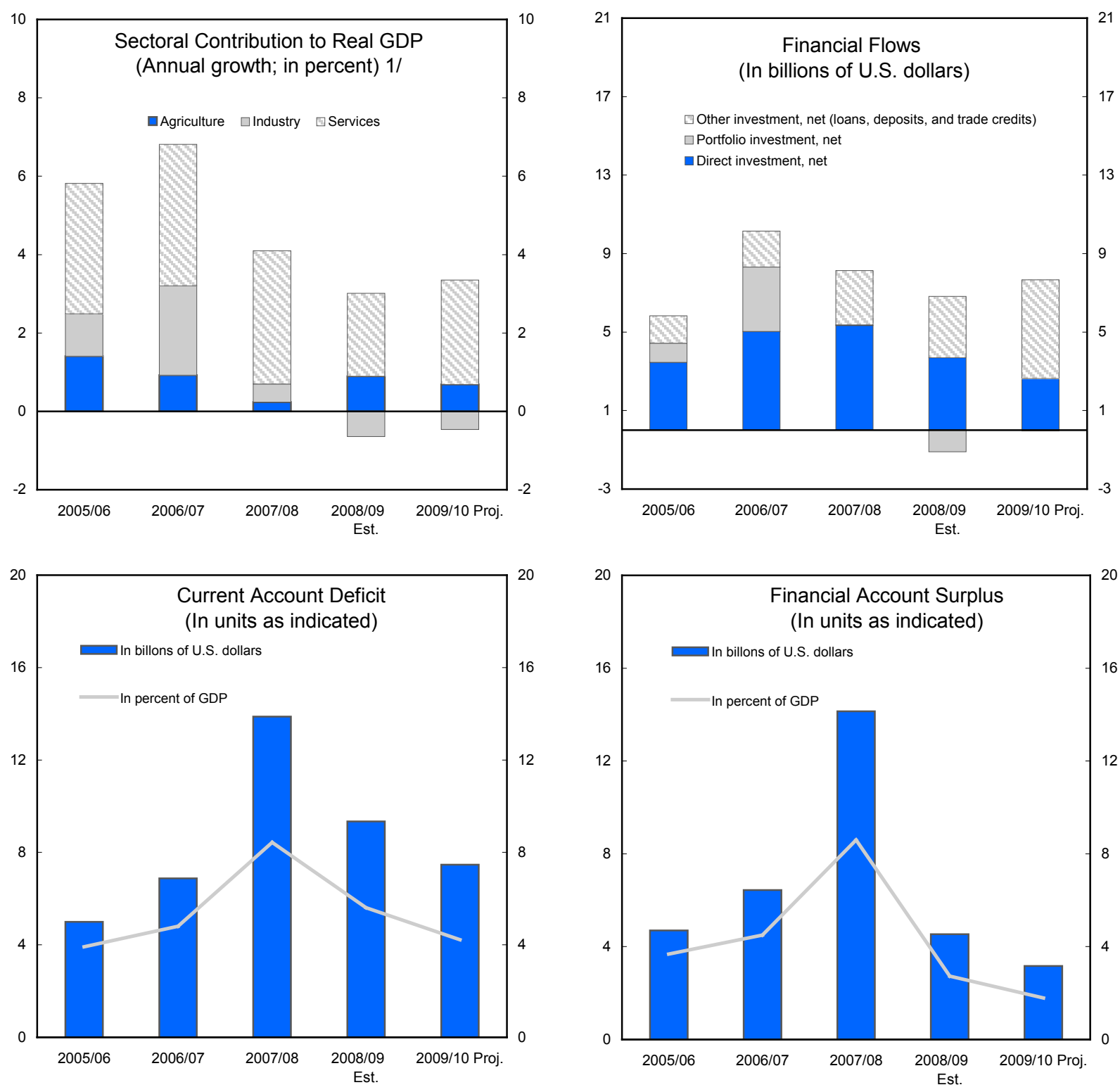

Sources: Pakistani authorities and Fund staff projections.

1/ At factor cost. 
Figure 4. Pakistan: Fiscal Policy Indicators, 2007/08-2009/10
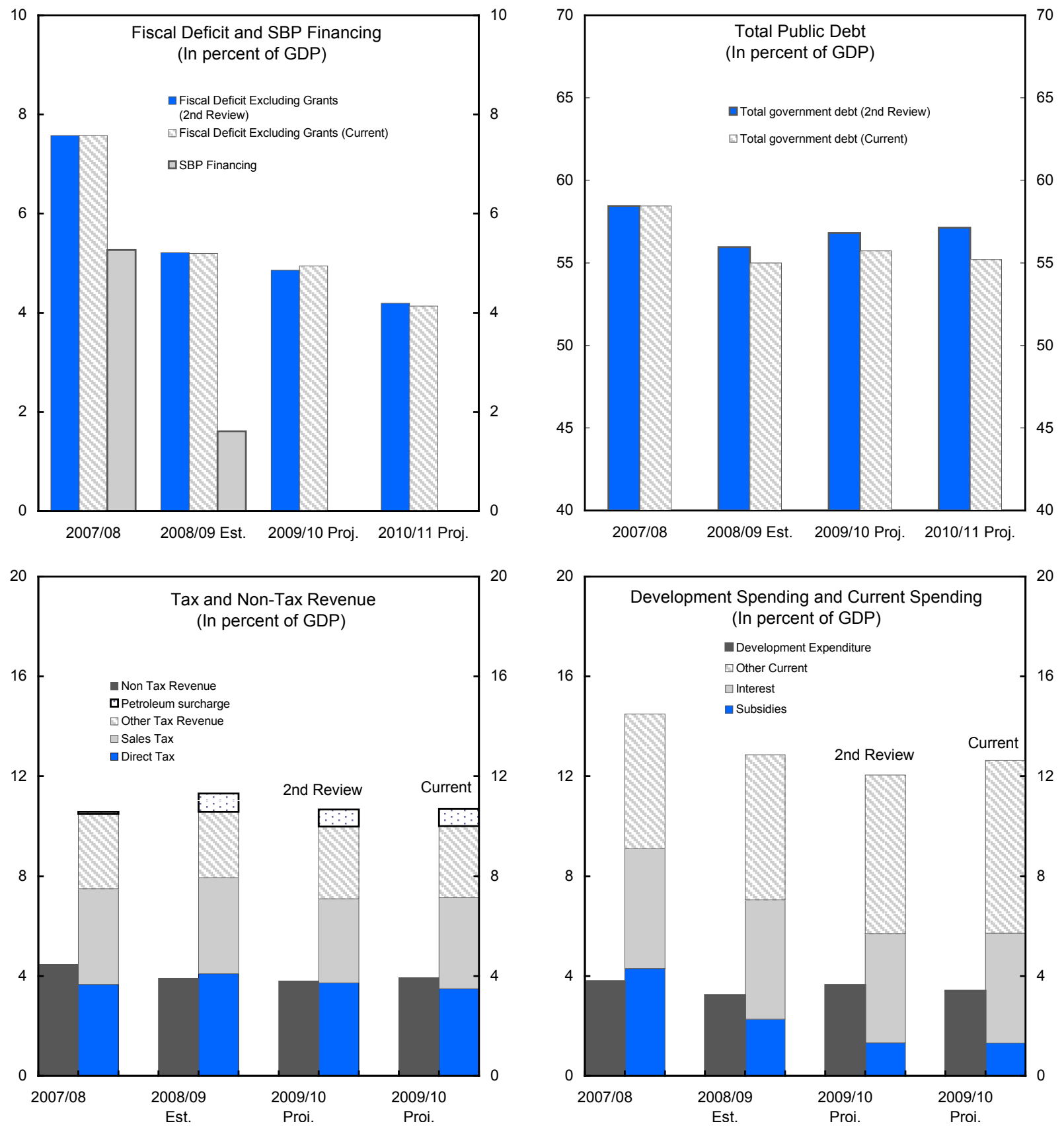

Sources: Pakistani authorities and Fund staff estimates and projections. 
Table 1. Pakistan: Selected Economic Indicators, 2007/08-2009/10 $1 /$

(Population: 160.9 million (2007/08))

(Per capita GDP: US\$1,042 (2007/08))

(Poverty rate: 23.9 percent $(2004 / 05))$

\begin{tabular}{|c|c|c|c|c|}
\hline & $2007 / 08$ & $\begin{array}{r}\text { Est. } \\
2008 / 09\end{array}$ & $\frac{\text { Rev. Prog. }}{200}$ & Proj. \\
\hline & \multicolumn{4}{|c|}{ (Annual percentage change) } \\
\hline \multicolumn{5}{|l|}{ Output and prices } \\
\hline Real GDP at factor cost & 4.1 & 2.0 & 3.0 & 3.0 \\
\hline Partner country demand (WEO definition) & 4.5 & & & \\
\hline Consumer prices (period average) & 12.0 & 20.8 & 10.0 & 11.0 \\
\hline Consumer prices (end of period) & 21.5 & 13.1 & 9.0 & 11.0 \\
\hline \multirow[t]{2}{*}{ Pakistani rupees per U.S. dollar (period average) } & 3.2 & 25.8 & $\ldots$ & $\ldots$ \\
\hline & \multicolumn{4}{|c|}{ (In percent of GDP) } \\
\hline Saving and investment & & & & \\
\hline Gross saving & 13.5 & 14.1 & 14.3 & 14.2 \\
\hline Government & -2.9 & -2.0 & -0.6 & -1.0 \\
\hline Nongovernment (including public sector enterprises) & 16.4 & 16.0 & 14.9 & 15.2 \\
\hline Gross capital formation 2/ & 22.0 & 19.7 & 19.0 & 18.5 \\
\hline Government & 4.4 & 3.0 & 3.6 & 3.3 \\
\hline Nongovernment (including public sector enterprises) & 17.6 & 16.7 & 15.5 & 15.1 \\
\hline \multicolumn{5}{|l|}{ Public finances } \\
\hline Revenue and grants & 14.9 & 14.3 & 15.2 & 15.2 \\
\hline Expenditure (including statistical discrepancy) & 22.2 & 19.3 & 19.3 & 19.6 \\
\hline Budget balance (including grants) & -7.3 & -5.0 & -4.2 & -4.3 \\
\hline Budget balance (including IDP grants) & $\ldots$ & $\ldots$ & -4.6 & -4.6 \\
\hline Budget balance (excluding grants) & -7.6 & -5.2 & -4.9 & -4.9 \\
\hline Primary balance & -2.5 & -0.1 & 0.2 & 0.1 \\
\hline Total government debt & 58.4 & 55.0 & 56.8 & 55.8 \\
\hline External government debt & 26.7 & 25.6 & 25.6 & 24.9 \\
\hline Domestic government debt & 31.8 & 29.4 & 31.3 & 30.9 \\
\hline & \multicolumn{4}{|c|}{$\begin{array}{l}\text { (Annual changes in percent of initial stock of broad } \\
\text { money, unless otherwise indicated) }\end{array}$} \\
\hline Monetary sector & & & & \\
\hline Net foreign assets & -7.8 & -3.2 & 1.9 & 3.0 \\
\hline Net domestic assets & 23.2 & 12.8 & 11.1 & 9.0 \\
\hline Broad money & 15.3 & 9.6 & 13.0 & 12.0 \\
\hline Private credit (percentage change) & 16.4 & 0.7 & 14.8 & 11.6 \\
\hline Six-month treasury bill rate (period average, in percent) & 9.6 & 13.1 & $\ldots$ & $\ldots$ \\
\hline \multicolumn{5}{|l|}{ External sector } \\
\hline Merchandise exports, U.S. dollars (percentage change) & 18.2 & -6.4 & -2.5 & -2.9 \\
\hline Merchandise imports, U.S. dollars (percentage change) & 31.2 & -10.3 & -2.4 & -2.5 \\
\hline Current account including official current transfers (in percent of GDP) & -8.4 & -5.6 & -4.7 & -4.2 \\
\hline & \multicolumn{4}{|c|}{$\begin{array}{c}\text { (In percent of exports of goods and services, } \\
\text { unless otherwise indicated) }\end{array}$} \\
\hline External public and publicly guaranteed debt & 167.6 & 177.8 & 194.1 & 185.6 \\
\hline Debt service & 15.1 & 21.2 & 19.9 & 21.2 \\
\hline Implicit interest rate (in percent) $3 /$ & 2.7 & 2.4 & 2.1 & 2.4 \\
\hline Gross reserves (in millions of U.S. dollars) $4 /$ & 8,591 & 9,114 & 13,474 & 14,104 \\
\hline In months of next year's imports of goods and services & 2.6 & 2.8 & 4.1 & 4.2 \\
\hline \multicolumn{5}{|l|}{ Memorandum items: } \\
\hline Real effective exchange rate (annual average, percentage change) & -0.8 & & $\ldots$ & $\ldots$ \\
\hline Terms of trade (percentage change) & -10.2 & 13.6 & & $\ldots$ \\
\hline Real per capita GDP (percentage change) & 2.3 & 0.3 & 1.3 & 1.3 \\
\hline GDP at market prices (in billions of Pakistani rupees) & 10,284 & 13,095 & 14,837 & 14,972 \\
\hline GDP at market prices (in billions of U.S. dollars) & 164.6 & 166.5 & 178.7 & 176.8 \\
\hline
\end{tabular}

Sources: Pakistani authorities; and Fund staff estimates and projections.

$1 /$ Fiscal year ends June 30 .

2/ Including changes in inventories. Investment data recorded by the Pakistan Federal Bureau of Statistics are said

to underreport true activity.

3/ Calculated as interest payments in percent of the end-of-period debt stock of the previous year.

4/ Excluding gold and foreign deposits of commercial banks held with the State Bank of Pakistan. 
Table 2. Pakistan: Balance of Payments, 2007/08-2009/10

(In millions of U.S. dollars; unless otherwise indicated)

\begin{tabular}{|c|c|c|c|c|c|c|c|c|}
\hline & \multirow[b]{3}{*}{$2007 / 08$} & \multirow[b]{3}{*}{$2008 / 09$} & Prel. & \multicolumn{3}{|c|}{ Projections } & \multirow{2}{*}{$\frac{\text { Rev. Prog. }}{\text { Yea }}$} & \multirow[t]{2}{*}{ Proj. } \\
\hline & & & Jul-Sep & Oct-Dec & Jan-Mar & Apr-Jun & & \\
\hline & & & \multicolumn{6}{|c|}{$2009 / 10$} \\
\hline Balance on goods & $-14,970$ & $-12,626$ & $-2,812$ & $-3,138$ & $-3,150$ & $-3,290$ & $-11,692$ & $-12,389$ \\
\hline Exports, f.o.b. & 20,427 & 19,121 & 4,630 & 4,600 & 4,650 & 4,682 & 18,441 & 18,562 \\
\hline Imports, f.o.b. & $-35,397$ & $-31,747$ & $-7,442$ & $-7,738$ & $-7,800$ & $-7,972$ & $-30,133$ & $-30,951$ \\
\hline Services (net) & $-6,457$ & $-3,473$ & -752 & $-1,037$ & -585 & -585 & $-2,454$ & $-2,959$ \\
\hline Services: debit & $-10,046$ & $-7,487$ & $-1,550$ & $-1,787$ & $-1,935$ & $-2,172$ & $-7,005$ & $-7,444$ \\
\hline Income (net) & $-3,923$ & $-4,403$ & -626 & $-1,178$ & $-1,150$ & $-1,662$ & $-4,333$ & $-4,616$ \\
\hline Income: credit & 1,613 & 873 & 106 & 203 & 230 & 345 & 909 & 883 \\
\hline Income: debit & $-5,536$ & $-5,276$ & -732 & $-1,381$ & $-1,380$ & $-2,006$ & $-5,241$ & $-5,499$ \\
\hline Of which: interest payments & $-2,161$ & $-1,908$ & -275 & -542 & -431 & -602 & $-1,850$ & $-1,850$ \\
\hline Of which: income on direct investment & $-3,375$ & $-3,368$ & -457 & -839 & -949 & $-1,404$ & $-3,391$ & $-3,649$ \\
\hline Balance on goods, services, and income & $-25,350$ & $-20,502$ & $-4,190$ & $-5,353$ & $-4,885$ & $-5,536$ & $-18,479$ & $-19,964$ \\
\hline Current transfers (net) & 11,476 & 11,163 & 3,611 & 3,433 & 3,163 & 2,285 & 9,992 & 12,492 \\
\hline Current transfers: credit, of which: & 11,618 & 11,265 & 3,645 & 3,446 & 3,180 & 2,280 & 10,050 & 12,550 \\
\hline Official & 484 & 219 & 2 & 46 & 155 & 98 & 464 & 300 \\
\hline Other private transfers & 4,683 & 3,235 & 1,312 & 1,100 & 900 & 438 & 2,886 & 3,750 \\
\hline Current transfers: debit & -142 & -102 & -34 & -13 & -16 & 5 & -58 & -58 \\
\hline Capital account & 121 & 474 & 33 & 24 & 461 & 628 & 964 & 1,146 \\
\hline Capital transfers: credit & 128 & 479 & 35 & 26 & 464 & 624 & 967 & 1,149 \\
\hline Of which: official capital grants & 111 & 427 & 27 & 22 & 460 & 625 & 952 & 1,134 \\
\hline Of which: Tokyo pledge & & $\ldots$ & 0 & 0 & 130 & 195 & 562 & 325 \\
\hline Capital transfers: debit & -7 & -5 & -2 & -2 & -3 & 4 & -3 & -3 \\
\hline Financial account & 8,131 & 5,720 & 2,095 & 575 & 2,039 & 2,919 & 7,625 & 7,630 \\
\hline Direct investment abroad & -75 & -25 & -36 & -15 & -20 & 41 & -30 & -30 \\
\hline Direct investment in Pakistan & 5,410 & 3,720 & 472 & 550 & 650 & 994 & 2,980 & 2,666 \\
\hline Of which: privatization receipts & 133 & 0 & 0 & 0 & 133 & 133 & 266 & 266 \\
\hline Portfolio investment (net), of which: & 32 & $-1,096$ & 202 & 150 & -475 & 89 & -484 & -34 \\
\hline Eurobond/GDR & -25 & -544 & -22 & 0 & -600 & 0 & -622 & -622 \\
\hline Other investment assets & 32 & 560 & -85 & -100 & -104 & 5 & -284 & -284 \\
\hline General government & 5 & 8 & 5 & 0 & -4 & -9 & -8 & -8 \\
\hline Banks & 525 & 346 & 61 & -50 & -50 & -161 & -200 & -200 \\
\hline Other sectors & -498 & 206 & -151 & -50 & -50 & 175 & -76 & -76 \\
\hline Disbursements & 3,054 & 3,642 & 616 & 272 & 1,741 & 1,819 & 5,408 & 4,448 \\
\hline Of which: Tokyo pledge & & & 0 & 0 & 900 & 276 & 1,928 & 1,176 \\
\hline Amortization & $-1,272$ & $-2,246$ & -528 & -957 & -360 & -332 & $-1,882$ & $-2,177$ \\
\hline Banks & 66 & 291 & 251 & 44 & 56 & -151 & 100 & 200 \\
\hline Other sectors & 415 & 302 & -47 & 631 & 551 & 454 & 550 & 1,591 \\
\hline Net errors and omissions & -166 & -89 & -521 & 0 & 0 & 0 & 0 & -522 \\
\hline Reserves and related items & 5,788 & 3,234 & $-1,028$ & 1,320 & -778 & -296 & -102 & -782 \\
\hline Reserve assets, of which: & 5,961 & -457 & $-2,150$ & 250 & $-1,900$ & $-1,340$ & $-4,460$ & $-5,140$ \\
\hline Foreign exchange (State Bank of Pakistan) & 5,711 & -523 & $-2,100$ & 300 & $-1,800$ & $-1,390$ & $-4,360$ & $-4,990$ \\
\hline Foreign exchange (deposit money banks) & 250 & 66 & -50 & -50 & -100 & 50 & -100 & -150 \\
\hline Use of Fund credit and loans & -173 & 3,691 & 1,122 & 1,070 & 1,122 & 1,044 & 4,358 & 4,358 \\
\hline Exceptional financing & 0 & 0 & 0 & 0 & 0 & 0 & 0 & 0 \\
\hline \multicolumn{9}{|l|}{ Memorandum items: } \\
\hline Current account (in percent of GDP) & -8.4 & -5.6 & -0.3 & -1.1 & -1.0 & -1.8 & -4.7 & -4.2 \\
\hline Current account (in percent of GDP; excluding fuel imports) & -2.1 & 0.4 & $\ldots$ & $\ldots$ & $\ldots$ & $\ldots$ & 1.0 & 1.9 \\
\hline Exports f.o.b. (growth rate, in percent) & 18.2 & -6.4 & $\ldots$ & $\ldots$ & $\ldots$ & $\ldots$ & -2.5 & -2.9 \\
\hline Imports f.o.b. (growth rate, in percent) & 31.2 & -10.3 & $\ldots$ & $\ldots$ & $\ldots$ & $\ldots$ & -2.4 & -2.5 \\
\hline Imports f.o.b. (growth rate, in percent, excluding oil) & 26.8 & -12.2 & $\ldots$ & $\ldots$ & $\ldots$ & $\ldots$ & -5.5 & -7.1 \\
\hline Oil imports (in million US\$, cif) & 10,496 & 10,031 & $\ldots$ & $\ldots$ & $\ldots$ & $\ldots$ & 10,333 & 10,888 \\
\hline Non-oil imports & 24,901 & 21,716 & & & & & 19,800 & 20,064 \\
\hline Crude oil price $(\$ / b b l)$ & 87.0 & 68.5 & $\ldots$ & $\ldots$ & $\ldots$ & $\ldots$ & 68.5 & 74.3 \\
\hline Terms of trade (growth rate, in percent) & -10.2 & $\ldots$ & $\ldots$ & $\ldots$ & $\ldots$ & $\ldots$ & $\ldots$ & $\ldots$ \\
\hline Workers' remittances and other private transfers & & & & & & & & \\
\hline (growth rate, in percent) & 10.1 & -0.8 & & & $\ldots$ & & -13.0 & 10.9 \\
\hline External debt (in millions of U.S. dollars) & 44,468 & 50,759 & 52,479 & 54,456 & 57,061 & 57,061 & 57,417 & 57,061 \\
\hline Gross financing needs (in millions of U.S. dollars) 1/ & 15,228 & 12,295 & $\ldots$ & $\ldots$ & $\ldots$ & $\ldots$ & 11,246 & 10,408 \\
\hline End-period gross official reserves (millions of U.S. dollars) 2/ & 8,591 & 9,114 & 11,214 & 10,914 & 12,714 & 14,104 & 13,474 & 14,104 \\
\hline (In months of next year's imports of goods and services) & 2.6 & 2.8 & $\ldots$ & $\ldots$ & $\ldots$ & $\ldots$ & 4.1 & 4.2 \\
\hline (in percent of debt service) & 244.4 & 187.4 & $\ldots$ & $\ldots$ & $\ldots$ & $\ldots$ & 292.3 & 294.7 \\
\hline GDP (in millions of U.S. dollars) & 164,557 & 166,515 & 176,835 & 176,835 & 176,835 & 176,835 & 178,723 & 176,835 \\
\hline
\end{tabular}

Sources: Pakistani authorities; and Fund staff estimates and projections.

1/ Defined as current account deficit, plus amortization on medium- and long-term debt, plus short-term debt at end of previous period.

2/ Excluding foreign currency deposits held with the State Bank of Pakistan (cash reserve requirements) and gold. 
Table 3a. Pakistan: Consolidated Government Budget, 2008/09-2009/10

(In billions of Pakistani rupees)

\begin{tabular}{|c|c|c|c|c|c|c|c|c|c|}
\hline & \multirow[t]{2}{*}{ Est. } & Rev. Progr. & Est. & \multicolumn{3}{|c|}{ Proj. } & \multirow[t]{3}{*}{ Budget } & \multirow[t]{3}{*}{ Rev. Progr. } & \multirow[t]{3}{*}{ Proj. } \\
\hline & & \multicolumn{2}{|c|}{ Jul-Sep } & Oct-Dec & Jan-Mar & Apr-Jun & & & \\
\hline & $2008 / 09$ & & & & & $2009 / 10$ & & & \\
\hline Revenue and grants & 1,877 & 525 & 428 & 555 & 604 & 694 & 2,222 & 2,251 & 2,280 \\
\hline Revenue & 1,851 & 490 & 426 & 540 & 568 & 654 & 2,156 & 2,146 & 2,187 \\
\hline Tax revenue & 1,331 & 334 & 305 & 399 & 398 & 498 & 1,593 & 1,583 & 1,600 \\
\hline Federal & 1,285 & 316 & 293 & 382 & 379 & 475 & 1,523 & 1,513 & 1,530 \\
\hline FBR revenue & 1,157 & 286 & 263 & 346 & 344 & 443 & 1,380 & 1,380 & 1,396 \\
\hline Direct taxes & 440 & 116 & 84 & 147 & 124 & 167 & 566 & 552 & 522 \\
\hline Federal excise duty & 116 & 30 & 28 & 41 & 40 & 52 & 153 & 171 & 161 \\
\hline Sales tax & 452 & 108 & 117 & 120 & 136 & 174 & 499 & 500 & 547 \\
\hline Customs duties & 148 & 32 & 33 & 39 & 44 & 50 & 162 & 156 & 166 \\
\hline Petroleum surcharge & 112 & 26 & 24 & 27 & 27 & 24 & 112 & 102 & 102 \\
\hline Gas surcharge and other & 16 & 5 & 7 & 9 & 8 & 8 & 31 & 31 & 31 \\
\hline Provincial & 46 & 17 & 11 & 17 & 19 & 23 & 70 & 70 & 70 \\
\hline Nontax revenue & 520 & 157 & 121 & 141 & 170 & 156 & 563 & 563 & 588 \\
\hline Federal & 436 & 142 & 113 & 115 & 140 & 125 & 468 & 468 & 493 \\
\hline Provincial & 84 & 15 & 8 & 26 & 30 & 31 & 95 & 95 & 95 \\
\hline Grants 1/ & 26 & 34 & 2 & 15 & 36 & 40 & 65 & 104 & 92 \\
\hline Of which: Tokyo pledges & $\ldots$ & 12 & 0 & 0 & 11 & 17 & 46 & 47 & 28 \\
\hline Of which: IDP grants & $\ldots$ & & 0 & 9 & 20 & 16 & 39 & 49 & 45 \\
\hline Expenditure & 2,497 & 684 & 636 & 715 & 772 & 791 & 2,877 & 2,867 & 2,913 \\
\hline Current expenditure & 2,093 & 576 & 544 & 593 & 625 & 641 & 2,261 & 2,327 & 2,403 \\
\hline Federal & 1,547 & 440 & 405 & 451 & 478 & 479 & 1,671 & 1,737 & 1,813 \\
\hline Interest & 638 & 149 & 141 & 172 & 159 & 188 & 647 & 649 & 659 \\
\hline Domestic & 559 & 134 & 129 & 145 & 140 & 163 & 577 & 577 & 577 \\
\hline Foreign & 79 & 15 & 12 & 27 & 19 & 25 & 70 & 72 & 83 \\
\hline Other $2 /$ & 909 & 291 & 263 & 279 & 319 & 292 & 1,024 & 1,088 & 1,153 \\
\hline Of which: Subsidies and grants & 380 & 159 & 126 & 142 & 161 & 111 & 446 & 510 & 540 \\
\hline Of which: Cash transfers to poor households $3 /$ & 21 & 13 & 13 & 17 & 25 & 29 & 84 & 84 & 84 \\
\hline Provincial & 546 & 136 & 140 & 142 & 148 & 161 & 590 & 590 & 590 \\
\hline Development expenditure and net lending & 404 & 109 & 92 & 122 & 147 & 150 & 617 & 540 & 510 \\
\hline Public Sector Development Program & 398 & 106 & 85 & 119 & 146 & 150 & 606 & 530 & 500 \\
\hline Federal & 196 & 66 & 54 & 69 & 94 & 83 & 406 & 330 & 300 \\
\hline Of which: One-off expenditure & 27 & 6 & 6 & 6 & 6 & 6 & 0 & 25 & 25 \\
\hline Provincial & 202 & 40 & 32 & 50 & 52 & 67 & 200 & 200 & 200 \\
\hline Net lending & 7 & 3 & 7 & 3 & 1 & 1 & 11 & 11 & 11 \\
\hline Statistical discrepancy ("+" = additional expenditure) 4/ & 34 & 0 & 14 & 0 & 0 & 0 & 0 & 0 & 14 \\
\hline Overall Deficit Ceiling (excluding grants) & -680 & -194 & -224 & -175 & -204 & -137 & -721 & -721 & -740 \\
\hline Overall Deficit (including IDP grants) & & -175 & -224 & -166 & -199 & -121 & $\ldots$ & -682 & -695 \\
\hline Overall Deficit (including grants) & -655 & -159 & -222 & -160 & -168 & -97 & -656 & -617 & -648 \\
\hline Financing & 655 & 159 & 222 & 160 & 168 & 97 & 656 & 617 & 648 \\
\hline External & 72 & 100 & 73 & -26 & 109 & 78 & 312 & 267 & 232 \\
\hline Of which: Tokyo pledges & $\ldots$ & 0 & 0 & 0 & 51 & 24 & $\ldots$ & 160 & 75 \\
\hline Of which: IMF & $\ldots$ & 59 & 61 & 30 & 30 & -55 & $\ldots$ & 0 & 66 \\
\hline Domestic & 583 & 60 & 149 & 186 & 59 & 19 & 343 & 350 & 415 \\
\hline Bank & 356 & 32 & 41 & 130 & 12 & -9 & 97 & 194 & 175 \\
\hline Nonbank & 227 & 28 & 108 & 56 & 47 & 28 & 246 & 156 & 240 \\
\hline \multicolumn{10}{|l|}{ Memorandum items: } \\
\hline Expenditure 5/ & 2,558 & 690 & 656 & 721 & 778 & 797 & 2,877 & 2,892 & 2,953 \\
\hline Primary balance (excluding grants) & -43 & -45 & -83 & -3 & -46 & 51 & -74 & -72 & -81 \\
\hline Primary balance (including grants) & -17 & -10 & -81 & 12 & -9 & 91 & -8 & 32 & 12 \\
\hline Augmented fiscal balance (excluding grants) 6/ & $\ldots$ & -410 & -440 & -236 & -204 & -137 & $\ldots$ & -998 & $-1,017$ \\
\hline Defense spending & 330 & 80 & 86 & 82 & 98 & 112 & 343 & 343 & 378 \\
\hline Total government debt 7 & 7,282 & $\ldots$ & $\ldots$ & $\ldots$ & & $\ldots$ & $\ldots$ & 8,430 & 8,439 \\
\hline Domestic debt & 3,853 & $\ldots$ & $\ldots$ & $\ldots$ & $\ldots$ & $\ldots$ & $\ldots$ & 4,637 & 4,632 \\
\hline External debt $7 /$ & 3,430 & & & $\ldots$ & & $\ldots$ & & 3,793 & 3,807 \\
\hline Nominal GDP (market prices) & 13,095 & 14,837 & 14,972 & 14,972 & 14,972 & 14,972 & 14,837 & 14,837 & 14,972 \\
\hline
\end{tabular}

Sources: Pakistani authorities for historical data; and Fund staff for estimates and projections.

1/ For 2009/10 projections, this figures includes unidentified IDP-grants in the amount of \$404 million.

2/ Includes Rs. 65 bn on security operations.

3/ Comprises BISP, Bait-ul-Mal, and Pakistan Poverty Alleviation Fund.

4/ The statistical discrepancy is believed to arise mainly from double-counting of spending at the provincial level.

$5 /$ Includes statistical discrepancy and spending related to the 2005 earthquake.

6/ Reflects assumption of electricity sector debt by the budget.

7/ Excludes military debt, commercial loans, and short-term debt. 
Table 3b. Pakistan: Consolidated Government Budget, 2008/09-2009/10

(In percent of GDP; unless otherwise indicated)

\begin{tabular}{|c|c|c|c|c|c|c|c|c|c|}
\hline & \multirow[t]{2}{*}{ Est. } & Rev. Progr. & Est. & \multicolumn{3}{|c|}{ Projections } & \multirow[t]{2}{*}{ Budget } & \multirow[t]{2}{*}{ Rev. Progr. } & \multirow[t]{3}{*}{ Proj. } \\
\hline & & \multicolumn{2}{|c|}{ Jul-Sep } & Oct-Dec & Jan-Mar & Apr-Jun & & & \\
\hline & $2008 / 09$ & & & \multicolumn{5}{|c|}{$2009 / 10$} & \\
\hline Revenue and grants & 14.3 & 3.5 & 2.9 & 3.7 & 4.0 & 4.6 & 15.0 & 15.2 & 15.2 \\
\hline Revenue & 14.1 & 3.3 & 2.8 & 3.6 & 3.8 & 4.4 & 14.5 & 14.5 & 14.6 \\
\hline Tax revenue & 10.2 & 2.2 & 2.0 & 2.7 & 2.7 & 3.3 & 10.7 & 10.7 & 10.7 \\
\hline Federal & 9.8 & 2.1 & 2.0 & 2.6 & 2.5 & 3.2 & 10.3 & 10.2 & 10.2 \\
\hline FBR revenue & 8.8 & 1.9 & 1.8 & 2.3 & 2.3 & 3.0 & 9.3 & 9.3 & 9.3 \\
\hline Direct taxes & 3.4 & 0.8 & 0.6 & 1.0 & 0.8 & 1.1 & 3.8 & 3.7 & 3.5 \\
\hline Federal excise duty & 0.9 & 0.2 & 0.2 & 0.3 & 0.3 & 0.3 & 1.0 & 1.2 & 1.1 \\
\hline Sales tax & 3.5 & 0.7 & 0.8 & 0.8 & 0.9 & 1.2 & 3.4 & 3.4 & 3.7 \\
\hline Customs duties & 1.1 & 0.2 & 0.2 & 0.3 & 0.3 & 0.3 & 1.1 & 1.1 & 1.1 \\
\hline Petroleum surcharge / Carbon tax & 0.9 & 0.2 & 0.2 & 0.2 & 0.2 & 0.2 & 0.8 & 0.7 & 0.7 \\
\hline Gas surcharge and other & 0.1 & 0.0 & 0.0 & 0.1 & 0.1 & 0.1 & 0.2 & 0.2 & 0.2 \\
\hline Provincial & 0.4 & 0.1 & 0.1 & 0.1 & 0.1 & 0.2 & 0.5 & 0.5 & 0.5 \\
\hline Nontax revenue & 4.0 & 1.1 & 0.8 & 0.9 & 1.1 & 1.0 & 3.8 & 3.8 & 3.9 \\
\hline Federal & 3.3 & 1.0 & 0.8 & 0.8 & 0.9 & 0.8 & 3.2 & 3.2 & 3.3 \\
\hline Provincial & 0.6 & 0.1 & 0.1 & 0.2 & 0.2 & 0.2 & 0.6 & 0.6 & 0.6 \\
\hline Grants 1/ & 0.2 & 0.2 & 0.0 & 0.1 & 0.2 & 0.3 & 0.4 & 0.7 & 0.6 \\
\hline Of which: Tokyo pledges & $\ldots$ & 0.1 & 0.0 & 0.0 & 0.1 & 0.1 & 0.3 & 0.3 & 0.2 \\
\hline Of which: IDP grants & & 0.0 & 0.0 & 0.1 & 0.1 & 0.1 & 0.3 & 0.3 & 0.3 \\
\hline Expenditure & 19.1 & 4.6 & 4.2 & 4.8 & 5.2 & 5.3 & 19.4 & 19.3 & 19.5 \\
\hline Current expenditure & 16.0 & 3.9 & 3.6 & 4.0 & 4.2 & 4.3 & 15.2 & 15.7 & 16.0 \\
\hline Federal & 11.8 & 3.0 & 2.7 & 3.0 & 3.2 & 3.2 & 11.3 & 11.7 & 12.1 \\
\hline Interest & 4.9 & 1.0 & 0.9 & 1.1 & 1.1 & 1.3 & 4.4 & 4.4 & 4.4 \\
\hline Domestic & 4.3 & 0.9 & 0.9 & 1.0 & 0.9 & 1.1 & 3.9 & 3.9 & 3.9 \\
\hline Foreign & 0.6 & 0.1 & 0.1 & 0.2 & 0.1 & 0.2 & 0.5 & 0.5 & 0.6 \\
\hline Other $2 /$ & 6.9 & 2.0 & 1.8 & 1.9 & 2.1 & 1.9 & 6.9 & 7.3 & 7.7 \\
\hline Of which: Subsidies and grants & 2.9 & 0.0 & 0.0 & 0.0 & 0.0 & 0.0 & 3.0 & 3.4 & 3.6 \\
\hline Of which: Cash transfers to poor households $3 /$ & 0.2 & $\ldots$ & $\ldots$ & $\ldots$ & $\ldots$ & $\ldots$ & 0.6 & 0.6 & 0.6 \\
\hline Provincial & 4.2 & 0.9 & 0.9 & 0.9 & 1.0 & 1.1 & 4.0 & 4.0 & 3.9 \\
\hline Development expenditure and net lending & 3.1 & 0.7 & 0.6 & 0.8 & 1.0 & 1.0 & 4.2 & 3.6 & 3.4 \\
\hline Public Sector Development Program & 3.0 & 0.7 & 0.6 & 0.8 & 1.0 & 1.0 & 4.1 & 3.6 & 3.3 \\
\hline Federal & 1.5 & 0.4 & 0.4 & 0.5 & 0.6 & 0.6 & 2.7 & 2.2 & 2.0 \\
\hline Of which: One-off expenditure & 0.2 & 0.0 & 0.0 & 0.0 & 0.0 & 0.0 & 0.0 & 0.2 & 0.2 \\
\hline Provincial & 1.5 & 0.3 & 0.2 & 0.3 & 0.3 & 0.4 & 1.3 & 1.3 & 1.3 \\
\hline Net lending & 0.1 & 0.0 & 0.0 & 0.0 & 0.0 & 0.0 & 0.1 & 0.1 & 0.1 \\
\hline Statistical discrepancy ("+" = additional expenditure) 4/ & 0.3 & 0.0 & 0.1 & 0.0 & 0.0 & 0.0 & 0.0 & 0.0 & 0.1 \\
\hline Overall Deficit (excluding grants) & -5.2 & -1.3 & -1.5 & -1.2 & -1.4 & -0.9 & -4.9 & -4.9 & -4.9 \\
\hline Overall Deficit (current proj. of IDP grants) & & 0.0 & 0.0 & 0.0 & 0.0 & 0.0 & -4.9 & -4.9 & -4.7 \\
\hline Overall Deficit (including IDP grants) & $\ldots$ & -1.2 & -1.5 & -1.1 & -1.3 & -0.8 & $\ldots$ & -4.6 & -4.6 \\
\hline Overall Deficit (including grants) & -5.0 & -1.1 & -1.5 & -1.1 & -1.1 & -0.6 & -4.4 & -4.2 & -4.3 \\
\hline Financing & 5.0 & 1.1 & 1.5 & 1.1 & 1.1 & 0.6 & 4.4 & 4.2 & 4.3 \\
\hline External & 0.5 & 0.7 & 0.5 & -0.2 & 0.7 & 0.5 & 2.1 & 1.8 & 1.6 \\
\hline Of which: Tokyo pledges & $\ldots$ & 0.0 & 0.0 & 0.0 & 0.3 & 0.2 & $\ldots$ & 1.1 & 0.5 \\
\hline Of which: IMF & $\ldots$ & 0.4 & 0.4 & 0.2 & 0.2 & -0.4 & $\ldots$ & 0.0 & 0.4 \\
\hline Domestic & 4.5 & 0.4 & 1.0 & 1.2 & 0.4 & 0.1 & 2.3 & 2.4 & 2.8 \\
\hline Bank & 2.7 & 0.2 & 0.3 & 0.9 & 0.1 & -0.1 & 0.7 & 1.3 & 1.2 \\
\hline Nonbank & 1.7 & 0.2 & 0.7 & 0.4 & 0.3 & 0.2 & 1.7 & 1.1 & 1.6 \\
\hline Privatization receipts & 0.0 & 0.0 & 0.0 & 0.0 & 0.0 & 0.0 & 0.0 & 0.0 & 0.0 \\
\hline \multicolumn{10}{|l|}{ Memorandum items: } \\
\hline Expenditure 5/ & 19.5 & 4.7 & 4.4 & 4.8 & 5.2 & 5.3 & 19.4 & 19.5 & 19.7 \\
\hline Primary balance (excluding grants) & -0.3 & -0.3 & -0.6 & 0.0 & -0.3 & 0.3 & -0.5 & -0.5 & -0.5 \\
\hline Primary balance (including grants) & -0.1 & -0.1 & -0.5 & 0.1 & -0.1 & 0.6 & -0.1 & 0.2 & 0.1 \\
\hline Augmented fiscal balance (excluding grants) $6 /$ & & -2.8 & -2.9 & -1.6 & -1.4 & -0.9 & $\ldots$ & -6.7 & -6.8 \\
\hline Defense spending & 2.5 & 0.5 & 0.6 & 0.5 & 0.7 & 0.7 & 2.3 & 2.3 & 2.5 \\
\hline Total government debt $7 /$ & 55.6 & $\ldots$ & $\ldots$ & $\ldots$ & $\ldots$ & $\ldots$ & $\ldots$ & 56.8 & 56.4 \\
\hline Domestic debt & 29.4 & $\ldots$ & $\ldots$ & $\ldots$ & $\ldots$ & $\ldots$ & $\ldots$ & 31.3 & 30.9 \\
\hline External debt $7 /$ & 26.2 & $\cdots$ & $\cdots$ & $\cdots$ & $\cdots$ & $\cdots$ & $\cdots$ & 25.6 & 25.4 \\
\hline Nominal GDP (market prices, billions of Pakistani rupees) & 13,095 & 14,837 & 14,972 & 14,972 & 14,972 & 14,972 & 14,837 & 14,837 & 14,972 \\
\hline
\end{tabular}

Sources: Pakistani authorities for historical data; and Fund staff for estimates and projections.

1/ For 2009/10 projections, this figures includes unidentified IDP-grants in the amount of $\$ 404$ million.

2/ Includes Rs 65 billion on security operations.

Alleviation Fund.

4/ The statistical discrepancy is believed to arise mainly from double-counting of spending at the provincial level.

$5 /$ Includes statistical discrepancy and spending related to the 2005 earthquake.

6/ Reflects assumption of electricity sector debt by the budget.

7/ Excludes military debt, commercial loans, and short-term debt. 
Table 4. Pakistan: Monetary Survey and Analytical Balance Sheet of the State Bank of Pakistan, 2007/08-2009/10

\begin{tabular}{|c|c|c|c|c|c|c|c|c|}
\hline & \multirow[b]{3}{*}{$2007 / 08$} & \multirow{3}{*}{$2008 / 09$} & \multirow{3}{*}{$\begin{array}{r}\text { Rev. Prog. } \\
\text { Sep. }\end{array}$} & \multirow[t]{3}{*}{ Act. } & \multicolumn{2}{|c|}{ Projections } & \multirow{3}{*}{$\begin{array}{c}\text { Rev. Prog. } \\
\text { Jun. }\end{array}$} & \multirow[t]{3}{*}{ Proj. } \\
\hline & & & & & \multirow{2}{*}{\multicolumn{2}{|c|}{$\frac{\text { Dec. }}{2009 / 10}$}} & & \\
\hline & & & & & & & & \\
\hline & \multicolumn{8}{|c|}{ (In billions of Pakistani rupees) } \\
\hline $\begin{array}{l}\text { Monetary survey } \\
\text { Net foreign assets (NFA) }\end{array}$ & & & & & & & & \\
\hline $\begin{array}{l}\text { Net foreign assets (NFA) } \\
\text { Net domestic assets (NDA) }\end{array}$ & 4,022 & 4,620 & $\begin{array}{r}5600 \\
4,695\end{array}$ & $\begin{array}{r}658 \\
4,517\end{array}$ & 4,851 & $\begin{array}{r}6,879 \\
4,879\end{array}$ & 5,189 & 5,082 \\
\hline Net claims on government, of which: & 1,473 & 1,997 & 2,008 & 2,032 & 2,155 & 2,104 & 2,188 & 2,121 \\
\hline Budget support, of which: & 1,325 & 1,630 & 1,663 & 1,669 & 1,799 & 1,811 & 1,825 & 1,805 \\
\hline Banks & 310 & 500 & 533 & 622 & 670 & 682 & 695 & 676 \\
\hline Commodity operations & 127 & 336 & 315 & 335 & 325 & 262 & 382 & 336 \\
\hline Credit to nongovernment & 3,018 & 3,190 & 3,280 & 3,180 & 3,400 & 3,488 & 3,722 & 3,680 \\
\hline $\begin{array}{l}\text { Private sector } \\
\text { Public sector enterorises }\end{array}$ & 2,904 & 2,924 & 2,989 & 2,849 & $\begin{array}{r}3,049 \\
{ }_{251}\end{array}$ & 3,113 & 3,357 & 3,263 \\
\hline $\begin{array}{l}\text { Public sector enterprises } \\
\text { Privatization account }\end{array}$ & $\begin{array}{r}114 \\
-3\end{array}$ & $\begin{array}{r}266 \\
-3\end{array}$ & $\begin{array}{r}291 \\
-3\end{array}$ & $\begin{array}{r}331 \\
-3\end{array}$ & $\begin{array}{r}351 \\
-3\end{array}$ & $\begin{array}{r}375 \\
-3\end{array}$ & $\begin{array}{r}365 \\
-3\end{array}$ & $\begin{array}{r}416 \\
-3\end{array}$ \\
\hline Other items, net & -466 & -564 & -590 & -692 & -701 & -710 & -717 & -716 \\
\hline Broad money & 4,689 & 5,137 & 5,255 & 5,176 & 5,422 & 5,553 & 5,806 & 5,753 \\
\hline Currency outside scheduled banks & 982 & 1,152 & 1,147 & 1,261 & 1,321 & 1,308 & 1,275 & 1,308 \\
\hline $\begin{array}{l}\text { Rupee deposits } \\
\text { Prepirn }\end{array}$ & 3,443 & 3,705 & 3,821 & $\begin{array}{r}3,614 \\
301\end{array}$ & 3,798 & 3,933 & 4,227 & $\begin{array}{r}4,126 \\
319\end{array}$ \\
\hline Foreign currency deposits & 263 & 280 & 286 & 301 & 303 & 313 & 303 & 319 \\
\hline \multicolumn{9}{|l|}{ State Bank of Pakistan (SBP) } \\
\hline NFA & 480 & 324 & 303 & 475 & 402 & 497 & 345 & 470 \\
\hline NDA & 1,000 & 1,183 & 1,256 & 1,141 & 1,318 & 1,228 & 1,346 & 1,262 \\
\hline $\begin{array}{l}\text { Net claims on government } \\
\text { Of }\end{array}$ & 1,015 & 1,144 & 1,144 & 1,058 & 1,144 & 1,144 & 1,144 & \\
\hline $\begin{array}{l}\text { Of which: budget support } \\
\text { Claims on nongovernment }\end{array}$ & 1,016 & 1,130 & 1,130 & 1,047 & 1,130 & 1,130 & 1,130 & 1,130 \\
\hline $\begin{array}{l}\text { Claims on nongovernment } \\
\text { Claims }\end{array}$ & -7 & -7 & -7 & -7 & -7 & -7 & -7 & \\
\hline $\begin{array}{l}\text { Claims on scheduled banks } \\
\text { Privatization account }\end{array}$ & 227 & 303 & 316 & 297 & 309 & 322 & 355 & 353 \\
\hline $\begin{array}{l}\text { Privatization account } \\
\text { Other items net }\end{array}$ & $\begin{array}{r}-3 \\
-231\end{array}$ & $\begin{array}{r}-3 \\
-254\end{array}$ & $\begin{array}{r}-3 \\
-194\end{array}$ & $\begin{array}{r}-3 \\
-204\end{array}$ & -3 & -32 & -115 & -32 \\
\hline $\begin{array}{l}\text { Other items, net } \\
\text { Reserve money of which: }\end{array}$ & 1.480 & 1.508 & 1.559 & 1.616 & 1.720 & 1.725 & $\begin{array}{l}-115 \\
1.690\end{array}$ & $\begin{array}{r}-226 \\
1.732\end{array}$ \\
\hline Banks' reserves & 425 & 274 & 281 & 267 & 310 & 328 & 333 & 341 \\
\hline Currency & 1,051 & 1,229 & 1,279 & 1,343 & 1,410 & 1,397 & 1,352 & 1,385 \\
\hline & \multicolumn{8}{|c|}{ (Annual percentage change, unless otherwise indicated) } \\
\hline Broad money & 15.3 & 9.6 & 12.4 & 10.7 & 13.1 & 15.6 & 13.0 & 12.0 \\
\hline NFA, banking system (in percent of broad money) $1 /$ & -7.8 & -3.2 & 1.6 & 3.7 & 4.1 & 4.4 & 1.9 & 3.0 \\
\hline NDA, banking system (in percent of broad money) $1 /$ & 23.2 & 12.8 & 10.8 & 7.0 & 9.1 & 11.2 & 11.1 & 9.0 \\
\hline & $\begin{array}{r}12.5 \\
32 ?\end{array}$ & 6.5 & 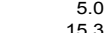 & 5.1 & 6.1 & $\begin{array}{r}6.4 \\
45.8\end{array}$ & 3.8 & 3.4 \\
\hline $\begin{array}{l}\text { NFA, banking system } \\
\text { NDA banking ssstem }\end{array}$ & $\begin{array}{l}-32.2 \\
30.6\end{array}$ & $\begin{array}{l}-22.5 \\
14.9\end{array}$ & $\begin{array}{l}15.3 \\
12.1\end{array}$ & $\begin{array}{r}35.6 \\
7.9\end{array}$ & 52.0 & $\begin{array}{ll}45.8 \\
12.3\end{array}$ & 19.2 & 29.8 \\
\hline $\begin{array}{l}\text { NDA, banking system } \\
\text { Budgetary support }\end{array}$ & $\begin{array}{l}30.6 \\
62.4\end{array}$ & $\begin{array}{l}14.9 \\
23.0\end{array}$ & $\begin{array}{l}12.1 \\
16.3\end{array}$ & $\begin{array}{r}7.9 \\
16.7\end{array}$ & $\begin{array}{r}9.8 \\
19.5\end{array}$ & $\begin{array}{l}12.3 \\
20.6\end{array}$ & $\begin{array}{l}12.3 \\
11.9\end{array}$ & $\begin{array}{l}10.0 \\
10.8\end{array}$ \\
\hline Private credit & 16.4 & 0.7 & -0.2 & -4.9 & -1.9 & 4.5 & 14.8 & 11.6 \\
\hline Currency & 16.9 & 17.3 & 3.6 & 13.9 & 17.5 & 17.3 & 10.7 & 13.6 \\
\hline Reserve money & 22.3 & 1.9 & 0.0 & 3.7 & 18.1 & 18.7 & 12.1 & 14.9 \\
\hline NFA, SBP (in percent of reserve money) $1 /$ & -25.4 & -10.5 & -0.3 & 10.7 & 14.8 & 16.9 & 1.3 & 9.7 \\
\hline NDA, SBP (in percent of reserve money) $1 /$ & 47.7 & 12.4 & 0.4 & -7.0 & 3.3 & 1.8 & 10.8 & 5.2 \\
\hline Net claims on government (in percent of reserve money) 1/ & 56.9 & 8.6 & -6.1 & -11.3 & -6.3 & 3.4 & 0.0 & 0.0 \\
\hline \multicolumn{9}{|l|}{ Memorandum items: } \\
\hline Velocity & 2.2 & 2.5 & & & & & 2.6 & 2.6 \\
\hline monerratio (nercent) & 3.2 & 3.4 & 3.4 & 3.2 & 3.2 & 3.2 & 3.4 & 3.3 \\
\hline ad money ratio (percent) & 20.9 & 22.4 & 21.8 & 24.4 & 24.4 & 23.5 & 22.0 & 22.7 \\
\hline Currency to d & 26.5 & 28.9 & 27.9 & 32.2 & 32.2 & 30.8 & 28.1 & 29.4 \\
\hline Reserves to deposit ratio (percent) & 11.5 & 6.9 & 6.8 & 6.8 & 7.5 & 7.7 & 7.4 & 7.7 \\
\hline Budget bank financing (billions of Pakistani rupees), of which: & 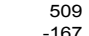 & 305 & 32 & $\begin{array}{r}39 \\
178\end{array}$ & $\begin{array}{l}169 \\
169\end{array}$ & $\begin{array}{l}181 \\
181\end{array}$ & $\begin{array}{l}194 \\
194\end{array}$ & $\begin{array}{l}175 \\
175\end{array}$ \\
\hline $\begin{array}{l}\text { By commercial banks } \\
\text { By SBP }\end{array}$ & $\begin{array}{r}-167 \\
677\end{array}$ & $\begin{array}{l}191 \\
114\end{array}$ & $\begin{array}{r}32 \\
0\end{array}$ & $\begin{array}{r}178 \\
-139\end{array}$ & 169 & $\begin{array}{r}181 \\
0\end{array}$ & $\begin{array}{r}194 \\
0\end{array}$ & 175 \\
\hline NFA of SBP (change from beginning of the year in billions of U.S. dollars) $2 /$ & -4.5 & -3.1 & -0.3 & $\begin{array}{r}-139 \\
1.7\end{array}$ & 0.7 & 1.8 & 0.1 & 1.4 \\
\hline NFA of commercial banks (billions of Pakistani rupees) & 187 & 193 & 257 & 184 & 169 & 177 & 272 & 201 \\
\hline NDA of commercial banks (billions of Pakistani rupees) & 3,022 & 3,437 & 3,439 & 3,376 & 3,533 & 3,651 & 3,844 & 3,820 \\
\hline Excess reserves in percent of broad money & 1.5 & 0.3 & & 0.0 & & & $\ldots$ & \\
\hline
\end{tabular}

Sources: Pakistani authorities for historical data; and Fund staff estimates and projections.

$1 /$ Denominator is the stock of broad (reserve) money at the end of the previous year.

2/ Includes valuation adjustments. 
Table 5. Pakistan: Medium-Term Macroeconomic Framework, 2007/08-2014/15

\begin{tabular}{|c|c|c|c|c|c|c|c|c|}
\hline & \multirow[b]{2}{*}{$2007 / 08$} & \multirow{2}{*}{$\frac{\text { Est. }}{2008 / 09}$} & \multicolumn{6}{|c|}{ Projections } \\
\hline & & & $2009 / 10$ & $2010 / 11$ & $2011 / 12$ & $2012 / 13$ & $2013 / 14$ & $2014 / 15$ \\
\hline & \multicolumn{8}{|c|}{ (In percent of GDP) } \\
\hline \multicolumn{9}{|l|}{ Saving and investment } \\
\hline Balance & -8.4 & -5.6 & -4.2 & -4.6 & -4.5 & -4.2 & -3.8 & -3.5 \\
\hline Government & -7.3 & -5.0 & -4.3 & -3.8 & -3.0 & -2.7 & -2.6 & -2.1 \\
\hline Non-government & -1.2 & -0.6 & 0.1 & -0.8 & -1.5 & -1.4 & -1.3 & -1.4 \\
\hline Gross national saving & 13.5 & 14.1 & 14.2 & 16.3 & 17.0 & 17.5 & 18.9 & 19.7 \\
\hline Government & -2.9 & -2.0 & -1.0 & 0.6 & 1.7 & 2.1 & 2.5 & 2.8 \\
\hline Non-government & 16.4 & 16.0 & 15.2 & 15.7 & 15.4 & 15.5 & 16.3 & 16.9 \\
\hline Gross capital formation & 22.0 & 19.7 & 18.5 & 20.9 & 21.5 & 21.7 & 22.7 & 23.2 \\
\hline Government & 4.4 & 3.0 & 3.3 & 4.4 & 4.7 & 4.8 & 5.1 & 4.9 \\
\hline Non-government (including public sector enterprises) & 17.6 & 16.7 & 15.1 & 16.5 & 16.9 & 16.9 & 17.6 & 18.3 \\
\hline & \multicolumn{8}{|c|}{ (In billions of U.S. dollars, unless otherwise indicated) } \\
\hline \multicolumn{9}{|l|}{ Balance of payments } \\
\hline Current account balance & -13.9 & -9.3 & -7.5 & -8.6 & -9.0 & -8.9 & -8.7 & -8.5 \\
\hline Net capital flows $1 /$ & 1.3 & 1.0 & 1.1 & 1.3 & 1.3 & 1.4 & 1.5 & 1.6 \\
\hline Of which: foreign direct investment 2/ & 5.4 & 3.7 & 2.7 & 3.5 & 4.0 & 4.5 & 5.2 & 5.7 \\
\hline Gross official reserves & 8.6 & 9.1 & 14.1 & 17.2 & 17.8 & 16.8 & 15.4 & 15.4 \\
\hline In months of imports 3 / & 2.6 & 2.8 & 4.2 & 4.8 & 4.7 & 4.2 & 3.6 & 3.5 \\
\hline \multirow[t]{2}{*}{ External debt (in percent of GDP) } & 27.0 & 29.6 & 31.4 & 33.2 & 33.4 & 32.2 & 30.1 & 28.2 \\
\hline & \multicolumn{8}{|c|}{ (In percent of GDP) } \\
\hline \multicolumn{9}{|l|}{ Public finances } \\
\hline Revenue and grants & 14.9 & 14.3 & 15.2 & 15.5 & 16.1 & 16.4 & 16.7 & 17.0 \\
\hline Of which: tax revenue & 10.6 & 10.2 & 10.7 & 11.4 & 12.2 & 12.7 & 13.0 & 13.4 \\
\hline Expenditure (incl. stat. discr.); of which: & 22.2 & 19.3 & 19.6 & 19.3 & 19.1 & 19.1 & 19.3 & 19.1 \\
\hline Current & 18.1 & 16.0 & 16.0 & 14.9 & 14.4 & 14.3 & 14.2 & 14.2 \\
\hline Development (incl. net-lending) & 4.1 & 3.1 & 3.4 & 4.4 & 4.7 & 4.8 & 5.1 & 4.9 \\
\hline Primary balance 4/ & -2.5 & -0.1 & 0.1 & 0.0 & 0.4 & 0.3 & 0.2 & 0.3 \\
\hline Overall fiscal balance 4/ & -7.3 & -5.0 & -4.3 & -3.8 & -3.0 & -2.7 & -2.6 & -2.1 \\
\hline \multirow[t]{2}{*}{ Total government debt } & 58.4 & 55.6 & 56.4 & 55.8 & 54.7 & 53.3 & 51.8 & 48.8 \\
\hline & \multicolumn{8}{|c|}{ (Annual changes in percent) } \\
\hline \multicolumn{9}{|l|}{ Output and prices } \\
\hline Real GDP at factor cost & 4.1 & 2.0 & 3.0 & 4.0 & 4.5 & 5.0 & 5.5 & 6.0 \\
\hline Consumer prices (period average) & 12.0 & 20.8 & 11.0 & 7.0 & 6.0 & 6.0 & 6.0 & 6.0 \\
\hline \multicolumn{9}{|l|}{ Memorandum item } \\
\hline Real per capita consumption (percentage change) & 5.6 & 7.5 & 1.0 & 2.5 & 2.5 & 2.5 & 2.5 & 2.5 \\
\hline
\end{tabular}

Sources: Pakistani authorities for historical data; and Fund staff estimates and projections.

$1 /$ Difference between the overall balance and the current account balance.

2/ Including privatization.

$3 /$ In months of next year's imports of goods and services.

4/ Including grants. 
Table 6. Pakistan: Medium-Term Balance of Payments, 2007/08-2014/15

(In millions of U.S. dollars; unless otherwise indicated)

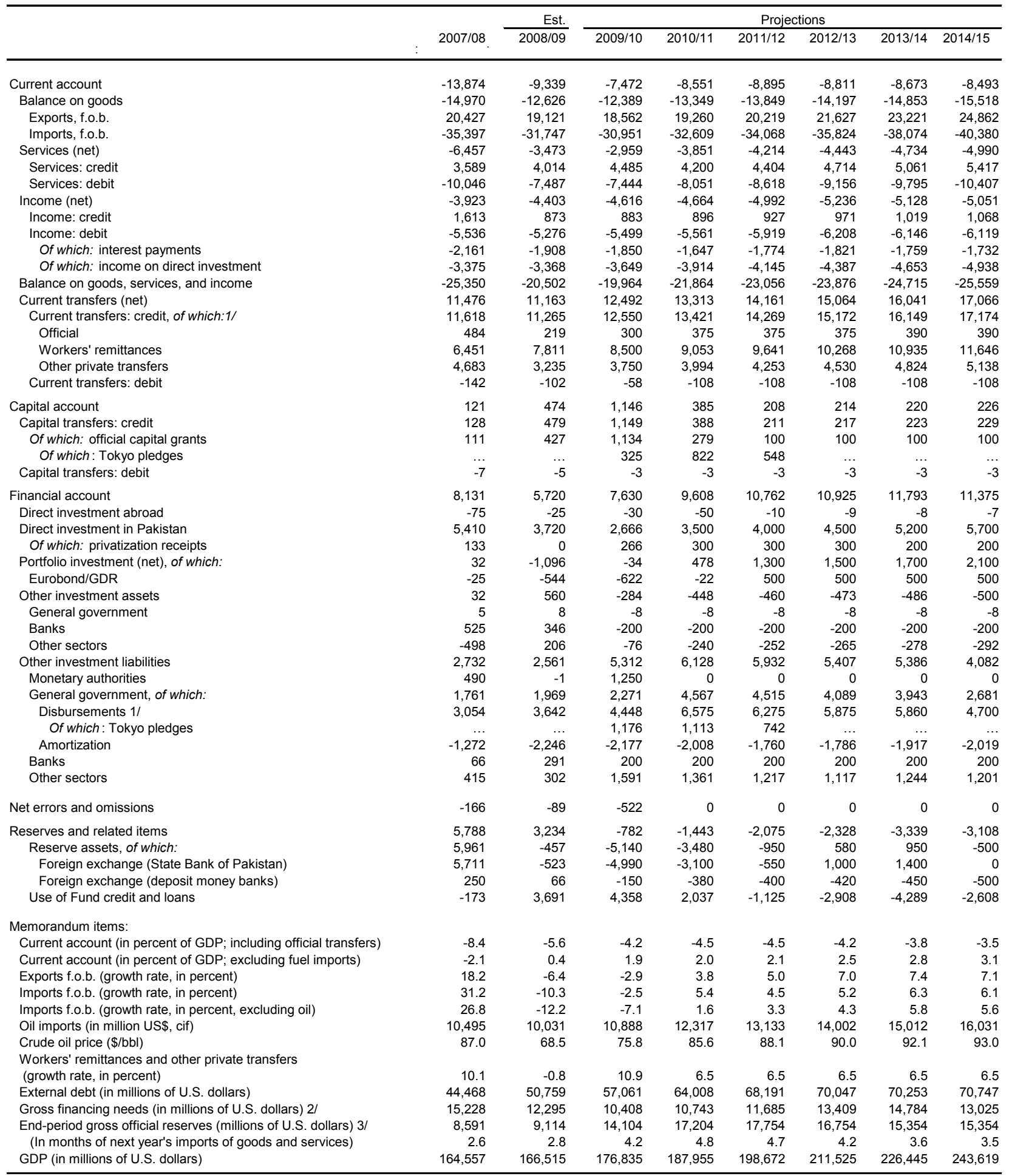

Sources: Pakistani authorities; and Fund staff estimates and projections.

1/ For 2010/11-2013/14, includes total U.S. Kerry-Lugar support of $\$ 7.5$ billon of which 15 percent assumed to be current transfers, and 85 percent as project support. 2/ Defined as current account deficit, plus amortization on medium- and long-term debt, plus short-term debt at end of previous period.

3/ Excluding foreign currency deposits held with the State Bank of Pakistan (cash reserve requirements) and gold. 
Table 7. Pakistan: Medium-Term Fiscal Framework, 2007/08-2014/15

(In percent of GDP; unless otherwise indicated)

\begin{tabular}{|c|c|c|c|c|c|c|c|c|}
\hline & \multirow[b]{2}{*}{$2007 / 08$} & \multirow{2}{*}{$\frac{\text { Est. }}{2008 / 09}$} & \multicolumn{6}{|c|}{ Projections } \\
\hline & & & $2009 / 10$ & $2010 / 11$ & $2011 / 12$ & $2012 / 13$ & $2013 / 14$ & $2014 / 15$ \\
\hline Revenue and grants & 14.9 & 14.3 & 15.2 & 15.5 & 16.1 & 16.4 & 16.7 & 17.0 \\
\hline Tax revenue & 10.6 & 10.2 & 10.7 & 11.4 & 12.2 & 12.7 & 13.0 & 13.4 \\
\hline Of which: Federal Board of Revenue & 9.8 & 8.8 & 9.3 & 10.1 & 10.9 & 11.5 & 11.9 & 12.3 \\
\hline Nontax revenue & 4.0 & 4.0 & 3.9 & 3.8 & 3.7 & 3.5 & 3.4 & 3.4 \\
\hline Grants & 0.3 & 0.2 & 0.6 & 0.3 & 0.2 & 0.2 & 0.2 & 0.2 \\
\hline Expenditure & 22.2 & 19.1 & 19.5 & 19.3 & 19.1 & 19.1 & 19.2 & 19.1 \\
\hline Current expenditure & 18.1 & 16.0 & 16.0 & 14.9 & 14.4 & 14.3 & 14.1 & 14.2 \\
\hline Interest & 4.8 & 4.9 & 4.4 & 3.8 & 3.4 & 3.0 & 2.7 & 2.4 \\
\hline Other federal & 9.1 & 6.9 & 7.7 & 6.7 & 6.5 & 6.7 & 6.9 & 7.2 \\
\hline Provincial & 4.3 & 4.2 & 3.9 & 4.4 & 4.5 & 4.6 & 4.6 & 4.6 \\
\hline Development expenditure & 4.4 & 3.0 & 3.3 & 4.4 & 4.7 & 4.8 & 5.1 & 4.9 \\
\hline Of which: one-off outlays $1 /$ & 0.2 & 0.2 & 0.2 & 0.0 & 0.0 & 0.0 & 0.0 & 0.0 \\
\hline Net-lending & -0.3 & 0.1 & 0.1 & 0.0 & 0.0 & 0.0 & 0.0 & 0.0 \\
\hline Statistical discrepancy & 0.0 & 0.3 & 0.1 & 0.0 & 0.0 & 0.0 & 0.0 & 0.0 \\
\hline \multicolumn{9}{|l|}{ Overall balance } \\
\hline Excluding grants & -7.6 & -5.2 & -4.9 & -4.1 & -3.2 & -3.0 & -2.8 & -2.3 \\
\hline Including grants & -7.3 & -5.0 & -4.3 & -3.8 & -3.0 & -2.7 & -2.5 & -2.1 \\
\hline Financing & 7.3 & 5.0 & 4.3 & 3.8 & 3.0 & 2.7 & 2.5 & 2.1 \\
\hline External & 1.2 & 0.5 & 1.6 & 2.6 & 2.7 & 2.3 & 2.1 & 2.2 \\
\hline Of which: privatization receipts & 0.0 & 0.0 & 0.2 & 0.2 & 0.2 & 0.1 & 0.1 & 0.1 \\
\hline Domestic & 6.1 & 4.5 & 2.8 & 1.2 & 0.3 & 0.4 & 0.5 & -0.1 \\
\hline \multicolumn{9}{|l|}{ Memorandum items: } \\
\hline \multicolumn{9}{|l|}{ Primary balance } \\
\hline Excluding grants & -2.8 & -0.3 & -0.5 & -0.4 & 0.2 & 0.1 & -0.1 & 0.1 \\
\hline Including grants & -2.5 & -0.1 & 0.1 & 0.0 & 0.4 & 0.3 & 0.2 & 0.3 \\
\hline Interest payments/revenue (ratio) & 32.7 & 34.5 & 30.2 & 24.7 & 21.4 & 18.8 & 16.4 & 14.2 \\
\hline Public debt $2 /$ & 58.4 & 55.6 & 56.4 & 55.8 & 54.7 & 53.3 & 51.8 & 48.8 \\
\hline External & 26.7 & 26.2 & 25.4 & 26.3 & 27.4 & 27.9 & 28.0 & 27.3 \\
\hline Domestic & 31.8 & 29.4 & 30.9 & 29.5 & 27.4 & 25.5 & 23.8 & 21.5 \\
\hline Public debt (including obligations to the IMF) & 59.3 & 58.8 & 61.9 & 62.1 & 60.1 & 57.0 & 53.2 & 49.1 \\
\hline Nominal GDP (billions of Pakistani rupees) & 10,284 & 13,095 & 14,972 & 16,660 & 18,455 & 20,540 & 22,970 & 25,809 \\
\hline
\end{tabular}

Sources: Pakistani authorities for historical data; and Fund staff for estimates and projections.

$1 /$ Spending related to the 2005 earthquake.

2/ Excludes obligations to the IMF, military debt, commercial loans, and short-term debt. 
Table 8. Pakistan: External Debt, 2007/08-2015/16

(In millions of U.S. dollars, outstanding at end of each fiscal year)

\begin{tabular}{|c|c|c|c|c|c|c|c|c|c|}
\hline & \multirow[b]{2}{*}{$2007 / 08$} & \multirow{2}{*}{$\frac{\text { Est. }}{2008 / 09}$} & \multicolumn{7}{|c|}{ Projections } \\
\hline & & & $2009 / 10$ & $2010 / 11$ & $2011 / 12$ & $2012 / 13$ & $2013 / 14$ & $2014 / 15$ & $2015 / 16$ \\
\hline Total external debt & 44,469 & 50,759 & 57,061 & 64,008 & 68,191 & 70,047 & 70,253 & 70,747 & 72,612 \\
\hline Public and publicly guaranteed debt (incl. IMF) & 41,582 & 47,259 & 53,266 & 59,849 & 63,739 & 65,421 & 65,575 & 66,148 & 68,063 \\
\hline Public and publicly guaranteed debt (excl. IMF) & 40,245 & 42,111 & 43,760 & 48,305 & 53,320 & 57,910 & 62,352 & 65,534 & 67,879 \\
\hline Medium- and long-term debt & 39,432 & 41,459 & 43,455 & 48,200 & 53,215 & 57,805 & 62,247 & 65,229 & 67,574 \\
\hline Multilateral creditors & 21,536 & 23,001 & 24,618 & 26,191 & 27,686 & 29,234 & 30,711 & 31,813 & 32,781 \\
\hline ADB & 9,401 & 9,035 & 10,041 & 11,219 & 12,325 & 13,256 & 14,132 & 14,960 & 15,753 \\
\hline World Bank & 11,483 & 12,017 & 12,639 & 13,084 & 13,528 & 14,204 & 14,871 & 15,221 & 15,469 \\
\hline Other & 651 & 1,949 & 1,938 & 1,887 & 1,833 & 1,774 & 1,707 & 1,633 & 1,559 \\
\hline Bilateral creditors & 15,181 & 15,947 & 17,309 & 20,504 & 23,523 & 26,064 & 28,530 & 29,909 & 31,287 \\
\hline Paris Club & 13,936 & 13,998 & 13,868 & 13,729 & 13,578 & 13,409 & 13,219 & 13,004 & 12,757 \\
\hline Non-paris club & 1,245 & 1,949 & 3,441 & 6,774 & 9,945 & 12,655 & 15,312 & 16,905 & 18,530 \\
\hline Bonds & 2,650 & 2,150 & 1,528 & 1,506 & 2,006 & 2,506 & 3,006 & 3,506 & 3,506 \\
\hline Of which: Euro bond & 2,650 & 2,150 & 1,528 & 1,506 & 2,006 & 2,506 & 3,006 & 3,506 & 3,506 \\
\hline Commercial banks and others & 65 & 361 & 0 & 0 & 0 & 0 & 0 & 0 & 0 \\
\hline Short-term debt & 813 & 652 & 305 & 105 & 105 & 105 & 105 & 305 & 305 \\
\hline Of which: IDB & 713 & 652 & 305 & 105 & 105 & 105 & 105 & 305 & 305 \\
\hline IMF & 1,337 & 5,148 & 9,506 & 11,543 & 10,418 & 7,511 & 3,222 & 614 & 184 \\
\hline Private sector debt & 2,887 & 3,500 & 3,795 & 4,159 & 4,453 & 4,626 & 4,679 & 4,599 & 4,549 \\
\hline \multicolumn{10}{|l|}{ Of which: } \\
\hline Multilateral creditors & 322 & $\ldots$ & $\ldots$ & $\ldots$ & $\ldots$ & $\ldots$ & $\ldots$ & $\ldots$ & $\ldots$ \\
\hline Paris Club creditors & 1,274 & $\ldots$ & $\ldots$ & $\ldots$ & $\ldots$ & $\ldots$ & $\ldots$ & $\ldots$ & $\ldots$ \\
\hline Non-paris club creditors & 9 & $\ldots$ & $\ldots$ & $\ldots$ & $\ldots$ & $\ldots$ & $\ldots$ & $\ldots$ & $\ldots$ \\
\hline Other & 1,007 & $\ldots$ & $\ldots$ & $\ldots$ & $\ldots$ & $\ldots$ & $\ldots$ & $\ldots$ & $\ldots$ \\
\hline
\end{tabular}

Sources: Pakistani authorities; and Fund staff estimates and projections. 
Table 9. Pakistan: Gross Balance of Payments Financing Requirements and Sources, 2007/08-2009/10

(In millions of U.S. dollars; unless otherwise specified)

\begin{tabular}{|c|c|c|c|c|}
\hline & \multirow[t]{2}{*}{$2007 / 08$} & $2008 / 09$ & $2009 / 10$ & $2010 / 11$ \\
\hline & & Prel. & \multicolumn{2}{|c|}{ Projections } \\
\hline Gross external financing requirements & 15,228 & 12,295 & 10,408 & 10,743 \\
\hline Current account deficit (if surplus $=-$ ) & 13,874 & 9,339 & 7,472 & 8,551 \\
\hline of which: net interest payments (if net receipts $=-$ ) & 2,161 & 1,908 & 1,850 & 1,647 \\
\hline Maturing short-term debt & 25 & 813 & 652 & 305 \\
\hline Amortization of medium- and long-term debt & 1,329 & 2,143 & 2,284 & 1,887 \\
\hline Medium and long-term to external private creditors & 25 & 544 & 622 & 22 \\
\hline Medium and long-term to external official creditors & 1,304 & 1,599 & 1,662 & 1,866 \\
\hline IMF & 173 & 211 & 232 & 258 \\
\hline To other official creditors & 1,131 & 1,388 & 1,430 & 1,608 \\
\hline World Bank (WB) & 422 & 392 & 398 & 398 \\
\hline Islamic Development Bank (IDB) & 20 & 200 & 20 & 20 \\
\hline Available financing & 15,228 & 12,295 & 10,408 & 10,743 \\
\hline Net FDI (including privatization receipts) & 5,335 & 3,695 & 2,636 & 3,450 \\
\hline Roll-over of short-term debt & 813 & 652 & 569 & -90 \\
\hline Medium- and long-term borrowing & 2,354 & 6,838 & 8,638 & 8,670 \\
\hline From external private creditors & 0 & 0 & 0 & 0 \\
\hline From external official creditors (currently identified) & 2,354 & 6,838 & 8,638 & 8,670 \\
\hline Borrowing from IMF (gross) & 0 & 3,902 & 4,590 & 2,295 \\
\hline Borrowing from other official creditors & 2,354 & 2,936 & 4,048 & 6,375 \\
\hline GDRs (public) & 0 & 0 & 0 & 0 \\
\hline Other net capital inflows & 1,015 & 1,633 & 3,555 & 1,813 \\
\hline Reserve accumulation (decrease $=+$ ) & 5,711 & -523 & $-4,990$ & $-3,100$ \\
\hline \multicolumn{5}{|l|}{ Memorandum items: } \\
\hline Gross international reserves in US\$ billions & 8.6 & 9.1 & 14.1 & 17.2 \\
\hline In months of imports & 2.6 & 2.8 & 4.2 & 4.8 \\
\hline
\end{tabular}

Sources: State Bank of Pakistan, and Fund staff estimates and projections. 
Table 10. Pakistan: Indicators of Fund Credit, 2008/09-2015/16

(In millions of SDR unless otherwise specified)

\begin{tabular}{|c|c|c|c|c|c|c|c|c|}
\hline & \multirow{2}{*}{$\begin{array}{r}\text { Est. } \\
2008 / 09\end{array}$} & \multicolumn{7}{|c|}{ Projections } \\
\hline & & $2009 / 10$ & $2010 / 11$ & $2011 / 12$ & $2012 / 13$ & $2013 / 14$ & $2014 / 15$ & $2015 / 16$ \\
\hline & \multicolumn{8}{|c|}{ (Projected Debt Service to the Fund based on Existing and Prospective Drawings) } \\
\hline \multicolumn{9}{|c|}{$\begin{array}{lllll} & \end{array}$} \\
\hline Principal $1 /$ & 137.7 & 155.1 & 172.3 & 163.7 & 120.6 & 51.7 & 17.2 & 0.0 \\
\hline Interest and charges $1 /$ & 4.0 & 3.1 & 2.2 & 1.4 & 0.6 & 0.2 & 0.0 & 0.0 \\
\hline \multicolumn{9}{|l|}{ Stand-By Arrangements } \\
\hline Principal & 0.0 & 0.0 & 0.0 & 587.9 & 1821.9 & 2813.7 & 1725.0 & 287.4 \\
\hline \multirow[t]{2}{*}{ Interest and charges } & 42.9 & 88.7 & 71.8 & 156.2 & 172.2 & 160.2 & 75.3 & 18.1 \\
\hline & \multicolumn{8}{|c|}{ (Projected Level of Credit Outstanding based on Existing and Prospective Drawings) } \\
\hline Total & $3,316.4$ & $6,228.2$ & $7,589.1$ & $6,837.5$ & $4,895.0$ & $2,029.6$ & 287.4 & 0.0 \\
\hline PRGF Arrangements & 680.5 & 525.4 & 353.2 & 189.5 & 69.0 & 17.3 & 0.0 & 0.0 \\
\hline \multirow[t]{2}{*}{ Stand-by Arrangements } & $2,635.9$ & $5,702.7$ & $7,235.9$ & $6,648.0$ & $4,826.0$ & $2,012.4$ & 287.4 & 0.0 \\
\hline & \multicolumn{8}{|c|}{ (Projected Debt Service to the Fund based on Existing and Prospective Drawings) } \\
\hline \multicolumn{9}{|l|}{ Of which: } \\
\hline Principal & 137.7 & 155.1 & 172.3 & 751.6 & $1,942.5$ & $2,865.4$ & $1,742.2$ & 287.4 \\
\hline Interest and charges & 46.9 & 91.7 & 74.0 & 157.6 & 172.8 & 160.3 & 75.3 & 18.1 \\
\hline In percent of exports of goods and non-factor services & 1.2 & 1.6 & 1.6 & 5.5 & 12.0 & 16.0 & 9.0 & 1.4 \\
\hline In percent of GDP & 0.2 & 0.2 & 0.2 & 0.7 & 1.5 & 2.0 & 1.1 & 0.2 \\
\hline In percent of end-period gross international reserves & 3.0 & 2.6 & 2.1 & 7.7 & 18.9 & 29.5 & 17.7 & 2.6 \\
\hline \multicolumn{9}{|l|}{ Memorandum items } \\
\hline Exports of goods and NFS (in millions of U.S. dollars) & 23,135 & 23,047 & 23,460 & 24,623 & 26,341 & 28,282 & 30,279 & 32,439 \\
\hline Quota & 1,034 & & & & & & & \\
\hline GDP (in millions of U.S. dollars) & 166,515 & 176,835 & 187,955 & 198,672 & 211,525 & 226,445 & 243,619 & 262,097 \\
\hline Fund credit outstanding (in percent of GDP) & 3.0 & 5.3 & 6.0 & 5.2 & 3.5 & 1.3 & 0.2 & 0.0 \\
\hline Of which: SBA & 2.4 & 4.8 & 5.8 & 5.0 & 3.4 & 1.3 & 0.2 & 0.0 \\
\hline Gross international reserves (in millions of U.S. dollars) & 9,114 & 14,104 & 17,204 & 17,754 & 16,754 & 15,354 & 15,354 & 17,454 \\
\hline
\end{tabular}

Source: Fund staff projections.

1/ For 2008/09, debt service includes payments related to EFF. 
Table 11. Pakistan: Access and Phasing under the Proposed Augmented Stand-By Arrangement, 2008-10

\begin{tabular}{|c|c|c|c|c|c|c|}
\hline \multirow[t]{2}{*}{ Review } & \multirow[t]{2}{*}{ Availability Date } & \multirow[t]{2}{*}{ Action } & \multicolumn{2}{|c|}{ Purchase } & \multicolumn{2}{|c|}{$\begin{array}{l}\text { Of which: for budget } \\
\text { financing }\end{array}$} \\
\hline & & & $\begin{array}{l}\text { In millions of } \\
\text { SDRs }\end{array}$ & $\begin{array}{l}\text { In percent } \\
\text { of quota }\end{array}$ & $\begin{array}{l}\text { In millions } \\
\text { of SDRs }\end{array}$ & $\begin{array}{c}\text { In percent } \\
\text { of quota }\end{array}$ \\
\hline & November 24, 2008 & Board approval of SBA & $2,067.400$ & 200.00 & $\ldots$ & $\ldots$ \\
\hline First Review & March 30, 2009 1/ & Observance of end-December 2008 performance criteria, completion of first review & 568.535 & 55.00 & $\ldots$ & $\ldots$ \\
\hline Second Review & August 7, 2009 2/ & Observance of end-June 2009 performance criteria, completion of second review & 766.700 & 74.17 & 475.60 & 46.01 \\
\hline Third Review & November 30, 2009 & Observance of end-September 2009 performance criteria, completion of third review & 766.700 & 74.17 & 237.75 & 23.00 \\
\hline Fourth Review & February 28,2010 & Observance of end-December 2009 performance criteria, completion of fourth review & 766.700 & 74.17 & 237.75 & 23.00 \\
\hline Fifth Review & May 31, 2010 & Observance of end-March 2010 performance criteria, completion of fifth review & 766.700 & 74.17 & $\ldots$ & $\ldots$ \\
\hline Sixth Review & August 31, 2010 & Observance of end-June 2010 performance criteria, completion of sixth review & 766.700 & 74.17 & $\ldots$ & $\ldots$ \\
\hline Seventh Review & November 30, 2010 & Observance of end-September 2010 performance criteria, completion of seventh review & 766.465 & 74.15 & $\ldots$ & $\ldots$ \\
\hline Total & & & $7,235.900$ & 700.00 & 951.10 & 92.01 \\
\hline
\end{tabular}

Source: Fund staff.

1/ The first review was completed on March 30 and the purchase was made on April 1.

2/ The second review was completed on August 7 and the purchase was made on August 11. 
Table 12. Pakistan: Selected Vulnerability Indicators, 2007/08-2014/15

\begin{tabular}{|c|c|c|c|c|c|c|c|c|}
\hline & $2007 / 08$ & $2008 / 09$ & $2009 / 10$ & $2010 / 11$ & $2011 / 12$ & $2012 / 13$ & $2013 / 14$ & $2014 / 15$ \\
\hline & & \multicolumn{7}{|c|}{ Projection } \\
\hline \multicolumn{9}{|l|}{ Key economic and market indicators } \\
\hline Real GDP growth (factor cost, in percent) & 4.1 & 2.0 & 3.0 & 4.0 & 4.5 & 5.0 & 5.5 & 6.0 \\
\hline CPI inflation (period average, in percent) & 12.0 & 20.8 & 11.0 & 7.0 & 6.0 & 6.0 & 6.0 & 6.0 \\
\hline Short-term (ST) interest rate (in percent) & 11.5 & $\ldots$ & $\ldots$ & $\ldots$ & $\ldots$ & $\ldots$ & $\ldots$ & $\ldots$ \\
\hline Emerging market bond index (EMBI) secondary market & & & & & & & & \\
\hline spread (basis points, end of period) $8 /$ & 687 & 1,039 & 632 & $\ldots$ & $\ldots$ & $\ldots$ & $\ldots$ & $\ldots$ \\
\hline Exchange rate PRs/US\$ (end of period) $8 /$ & 68.2 & 81.4 & 83.6 & $\ldots$ & $\ldots$ & $\ldots$ & $\ldots$ & $\ldots$ \\
\hline \multicolumn{9}{|l|}{ External sector } \\
\hline Exchange rate regime & \multicolumn{8}{|c|}{ Floating since January 2008} \\
\hline Current account balance (percent of GDP) & -8.4 & -5.6 & -4.2 & -4.6 & -4.5 & -4.2 & -3.8 & -3.5 \\
\hline Net FDI inflows (percent of GDP) & 3.2 & 2.2 & 1.5 & 1.8 & 2.0 & 2.1 & 2.3 & 2.3 \\
\hline Exports (percentage change of U.S. dollar value; GNFS) & 12.1 & -3.7 & -0.4 & 1.8 & 5.0 & 7.0 & 7.4 & 7.1 \\
\hline Real effective exchange rate $(2000=100$, annual average $)$ & 97.1 & $\ldots$ & & $\ldots$ & $\ldots$ & $\ldots$ & $\ldots$ & \\
\hline Gross international reserves (GIR) in billions of U.S. dollars & 8.6 & 9.1 & 14.1 & 17.2 & 17.8 & 16.8 & 15.4 & 15.4 \\
\hline GIR in percent of ST debt at remaining maturity (RM) 1/ & 436.3 & 365.4 & 625.7 & 986.7 & $1,060.5$ & 985.8 & 838.5 & 689.6 \\
\hline GIR in percent of ST debt at RM and banks' foreign exchange ( $F X)$ deposits $1 /$ & 147.3 & 153.5 & 238.3 & 297.1 & 297.3 & 266.6 & 227.6 & 204.2 \\
\hline Total gross external debt (ED) in percent of GDP, of which: & 27.0 & 29.6 & 31.4 & 33.2 & 33.4 & 32.2 & 30.1 & 28.2 \\
\hline ST external debt (original maturity, in percent of total ED) & 1.8 & 1.3 & 0.5 & 0.2 & 0.2 & 0.2 & 0.2 & 0.4 \\
\hline ED of domestic private sector (in percent of total ED) & 6.5 & 6.4 & 6.2 & 5.9 & 5.8 & 5.8 & 5.6 & 5.5 \\
\hline ED to foreign official sector (in percent of total ED) & 93.5 & 93.6 & 93.8 & 94.1 & 94.2 & 94.2 & 94.4 & 94.5 \\
\hline Total gross external debt in percent of exports & 185.2 & 212.9 & 241.1 & 265.9 & 269.9 & 258.8 & 241.3 & 226.8 \\
\hline Gross external financing requirement (in billions of U.S. dollars) 2/ & 15.2 & 12.3 & 10.4 & 10.7 & 11.7 & 13.4 & 14.8 & 13.0 \\
\hline Public sector $3 /$ & \multicolumn{8}{|c|}{ (In percent of GDP) } \\
\hline Overall balance (including grants) & -7.3 & -5.0 & -4.3 & -3.8 & -3.0 & -2.7 & -2.6 & -2.1 \\
\hline Primary balance (including grants) & -2.5 & -0.1 & 0.1 & 0.0 & 0.4 & 0.3 & 0.2 & 0.3 \\
\hline Debt-stabilizing primary balance $4 /$ & -1.4 & -3.6 & -1.2 & -1.0 & -1.0 & -1.5 & -1.9 & 0.0 \\
\hline Gross PS financing requirement $5 /$ & 24.7 & 22.0 & 21.7 & 19.8 & 17.8 & 16.6 & 15.5 & 13.9 \\
\hline Public sector gross debt & 58.4 & 55.6 & 56.4 & 55.8 & 54.7 & 53.3 & 51.8 & 48.8 \\
\hline Public sector net debt $6 /$ & 53.8 & 50.4 & 51.2 & 50.6 & 49.6 & 48.3 & 46.8 & 43.8 \\
\hline \multicolumn{9}{|l|}{ Financial sector $7 /$} \\
\hline Capital adequacy ratio (in percent) $9 /$ & 12.1 & 13.5 & 14.3 & $\ldots$ & $\ldots$ & $\ldots$ & $\ldots$ & $\ldots$ \\
\hline Nonperforming loans (NPLs) in percent of total loans 9/ & 7.7 & 11.5 & 12.4 & $\ldots$ & $\ldots$ & $\ldots$ & $\ldots$ & $\ldots$ \\
\hline Provisions in percent of NPLs $9 /$ & 84.0 & 70.2 & 69.7 & $\ldots$ & $\ldots$ & $\ldots$ & $\ldots$ & $\ldots$ \\
\hline Return on average assets (after tax, in percent) 9/ & 1.7 & 1.0 & 0.9 & $\ldots$ & $\ldots$ & $\ldots$ & $\ldots$ & $\ldots$ \\
\hline Return on equity (after tax, in percent) $9 /$ & 16.7 & 9.7 & 9.0 & $\ldots$ & $\ldots$ & $\ldots$ & $\ldots$ & $\ldots$ \\
\hline FX deposits held by residents (in percent of total deposits) & 7.1 & 7.0 & 7.2 & $\ldots$ & $\ldots$ & $\ldots$ & $\ldots$ & $\ldots$ \\
\hline Government debt held by FS (percent of total FS assets) & 31.4 & 38.9 & 36.9 & $\ldots$ & $\ldots$ & $\ldots$ & $\ldots$ & $\ldots$ \\
\hline Credit to private sector (percent change) & 16.4 & 0.7 & 11.6 & $\ldots$ & $\ldots$ & $\ldots$ & $\ldots$ & $\ldots$ \\
\hline \multicolumn{9}{|l|}{ Memorandum item: } \\
\hline Nominal GDP (in billions of U.S. dollars) & 164.6 & 166.5 & 176.8 & 188.0 & 198.7 & 211.5 & 226.4 & 243.6 \\
\hline
\end{tabular}

Sources: Pakistani authorities; and Fund staff estimates and projections.

$1 /$ Debt at remaining maturity is defined as maturing short-, medium-, and long-term external official debt.

2/ Current account deficit plus amortization of external debt.

3/ Public sector covers general (consolidated) government.

4/ Based on the end of period debt stock in year t-1, and the baseline assumptions for the relevant variables (i.e., growth, interest rates,

inflation, exchange rates) in year $\mathrm{t}$.

5/ Overall balance plus debt amortization.

6/ Net debt is defined as gross debt minus government deposits with the banking system.

7/ Financial sector includes all commercial and specialized banks; for government debt also includes nonbanks, but excludes State Bank of Pakistan.

$8 / 2009 / 10$ is end-November data.

$9 / 2009 / 10$ is end-September data. 
Table 13. Pakistan: Financial Soundness Indicators for the Banking System

(December 2005-September 2009)

\begin{tabular}{|c|c|c|c|c|c|c|c|}
\hline & $\begin{array}{l}\text { Dec. } \\
2005\end{array}$ & $\begin{array}{l}\text { Dec. } \\
2006\end{array}$ & $\begin{array}{r}\text { Dec. } \\
20071 /\end{array}$ & $\begin{array}{r}\text { Dec. } \\
20081 /\end{array}$ & $\begin{array}{l}\text { Mar. } \\
2009\end{array}$ & $\begin{array}{l}\text { Jun. } \\
2009\end{array}$ & $\begin{array}{l}\text { Sep. } \\
2009\end{array}$ \\
\hline \multicolumn{8}{|l|}{ Capital adequacy } \\
\hline Regulatory capital to risk-weighted assets & 11.3 & 12.7 & 12.3 & 12.3 & 12.9 & 13.5 & 14.3 \\
\hline Tier I capital to risk-weighted assets & 8.3 & 10.0 & 10.0 & 10.2 & 10.8 & 11.3 & \\
\hline Capital to total assets & 7.9 & 9.4 & 10.5 & 10.0 & 10.3 & 10.2 & 10.5 \\
\hline \multicolumn{8}{|l|}{ Asset composition and quality } \\
\hline Nonperforming loans (NPLs) to gross loans & 8.3 & 6.9 & 7.6 & 10.5 & 11.5 & 11.5 & 12.4 \\
\hline Provisions to NPLs & 76.7 & 77.8 & 86.1 & 69.6 & 69.2 & 70.2 & 69.7 \\
\hline NPLs net of provisions to capital & 14.3 & 9.7 & 5.6 & 19.4 & 19.6 & 19.0 & 19.9 \\
\hline \multicolumn{8}{|l|}{ Earnings and profitability } \\
\hline Return on assets (after tax) & 1.9 & 2.1 & 1.5 & 0.8 & 1.1 & 1.0 & 0.9 \\
\hline Return on equity (after tax) & 25.8 & 23.8 & 15.4 & 7.8 & 10.7 & 9.7 & 9.0 \\
\hline Net interest income to gross income & 72.0 & 70.9 & 68.2 & 70.4 & 75.8 & 73.0 & 73.1 \\
\hline Noninterest expenses to gross income & 41.5 & 40.3 & 43.2 & 50.3 & 49.9 & 50.1 & 50.1 \\
\hline \multicolumn{8}{|l|}{ Liquidity } \\
\hline Liquid assets to total assets & 33.7 & 31.9 & 33.6 & 28.6 & 30.5 & 31.2 & 31.4 \\
\hline Liquid assets to total deposits & 43.5 & 42.7 & 45.1 & 38.2 & 41.5 & 41.7 & 42.7 \\
\hline
\end{tabular}

Source: State Bank of Pakistan.

1/ Data for 2007 and 2008 have been restated on the basis of annual audits. 


\section{ApPendix. Pakistan: Debt Sustainability Analysis}

Figure 1. Pakistan: External Debt Sustainability-Bound Tests 1/

(External debt in percent of GDP)
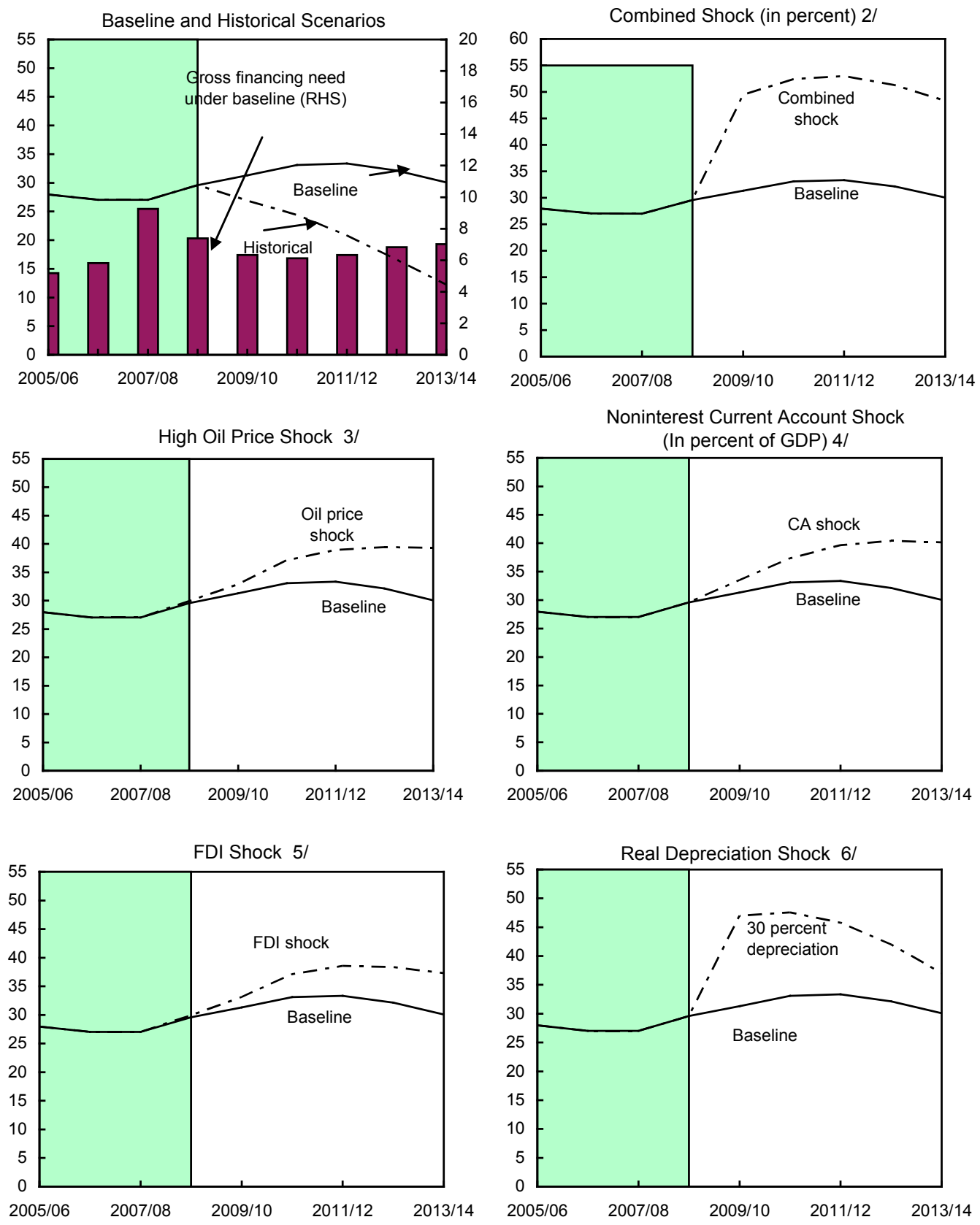

Sources: Historical data from Pakistani authorities; and Fund staff estimates and projections,

1/ Shaded areas represent actual data. Individual shocks are permanent one-half standard deviation shocks. Figures in the boxes represent average projections for the respective variables in the baseline and scenario being presented. Ten-year historical average for the variable is also shown.

2/ Combined impact of shocks to growth, current account, FDI, and real depreciation.

$3 /$ Oil prices are higher by $\$ 10$ for the projection period relative to the baseline.

4/ Current account deficit higher than the baseline scenario by half of the ten-year standard deviation.

$5 /$ Net flows of foreign direct investment are 50 percent lower than in the baseline scenario.

$6 /$ One-time real depreciation of 30 percent occurs in 2009/10. 
Table 1. Pakistan: External Debt Sustainability Framework, 2004/05-2013/14

(In percent of GDP, unless otherwise indicated)

\begin{tabular}{|c|c|c|c|c|c|c|c|c|c|c|c|}
\hline \multirow[b]{3}{*}{ Baseline: External debt } & \multicolumn{4}{|c|}{ Actual } & \multicolumn{6}{|c|}{ Projections } & \multirow{2}{*}{$\begin{array}{c}\text { Debt-stabilizing } \\
\text { noninterest current } \\
\text { account } 71\end{array}$} \\
\hline & $2004 / 05$ & $2005 / 06$ & $2006 / 07$ & $2007 / 08$ & $2008 / 09$ & $2009 / 10$ & $2010 / 11$ & $2011 / 12$ & $2012 / 13$ & $2013 / 14$ & \\
\hline & 31.1 & 28.0 & 27.0 & 27.0 & 30.5 & 32.3 & 34.1 & 34.3 & 33.1 & 31.0 & -4.2 \\
\hline Change in external debt & -3.0 & -3.1 & -0.9 & 0.0 & 3.5 & 1.8 & 1.8 & 0.3 & -1.2 & -2.1 & \\
\hline Identified external debt-creating flows $(4+8+9)$ & -3.7 & -3.5 & -3.5 & 1.6 & 3.2 & 1.5 & 1.2 & 0.6 & 0.0 & -0.7 & \\
\hline Current account deficit, excluding interest payments & 0.5 & 2.9 & 3.8 & 7.1 & 4.5 & 3.2 & 3.7 & 3.6 & 3.3 & 3.1 & \\
\hline Deficit in balance of goods and services & 7.1 & 10.1 & 9.7 & 13.0 & 9.7 & 8.7 & 9.2 & 9.1 & 8.8 & 8.6 & \\
\hline Exports & 16.2 & 15.9 & 15.0 & 14.6 & 13.9 & 13.0 & 12.5 & 12.4 & 12.5 & 12.5 & \\
\hline Imports & 23.4 & 26.0 & 24.6 & 27.6 & 23.6 & 21.7 & 21.6 & 21.5 & 21.3 & 21.1 & \\
\hline Net nondebt-creating capital inflows (negative) & -1.5 & -3.1 & -5.2 & -3.3 & -1.9 & -1.8 & -2.1 & -2.4 & -2.6 & -2.8 & \\
\hline Automatic debt dynamics $1 /$ & -2.6 & -3.4 & -2.1 & -2.2 & 0.6 & 0.2 & -0.3 & -0.6 & -0.8 & -0.9 & \\
\hline Contribution from nominal interest rate & 0.9 & 1.0 & 1.0 & 1.3 & 1.1 & 1.0 & 0.9 & 0.9 & 0.9 & 0.8 & \\
\hline Contribution from real GDP growth & -2.3 & -1.6 & -1.4 & -0.5 & -0.5 & -0.9 & -1.2 & -1.4 & -1.6 & -1.7 & \\
\hline Contribution from price and exchange rate changes $2 /$ & -1.2 & -2.7 & -1.7 & -3.0 & $\ldots$ & & & $\ldots$ & & $\ldots$ & \\
\hline Residual, incl. change in gross foreign assets $(2-3) 3 /$ & 0.7 & 0.4 & 2.5 & -1.6 & 0.3 & 0.3 & 0.6 & -0.3 & -1.2 & -1.4 & \\
\hline External debt-to-exports ratio (in percent) & 191.2 & 175.5 & 180.7 & 185.2 & 219.4 & 247.6 & 272.8 & 276.9 & 265.9 & 248.4 & \\
\hline Gross external financing need (in billions of US dollars) 4 & 3.5 & 6.6 & 8.3 & 15.2 & 12.3 & 10.4 & 10.7 & 11.7 & 13.4 & 14.8 & \\
\hline In percent of GDP & 3.2 & 5.2 & 5.8 & 9.3 & 7.4 & 5.9 & 5.7 & 5.9 & 6.3 & 6.5 & \\
\hline Scenario with key variables at their historical averages 5 / & $\ldots$ & $\ldots$ & $\ldots$ & $\ldots$ & 30.5 & 28.2 & 26.1 & 22.9 & 19.2 & 15.3 & -2.3 \\
\hline \multicolumn{12}{|l|}{ Key Macroeconomic Assumptions Underlying Baseline } \\
\hline Real GDP growth (in percent) & 7.7 & 6.1 & 5.6 & 2.0 & 2.0 & 3.0 & 4.0 & 4.5 & 5.0 & 5.5 & \\
\hline GDP deflator in US dollars (change in percent) & 3.8 & 9.6 & 6.3 & 12.6 & -0.8 & 3.1 & 2.2 & 1.2 & 1.4 & 1.5 & \\
\hline Nominal external interest rate (in percent) & 3.1 & 3.7 & 4.0 & 5.6 & 4.3 & 3.6 & 2.9 & 2.8 & 2.7 & 2.5 & \\
\hline Growth of exports of goods and services (U.S. dollar terms, in percent) 6/ & 17.9 & 14.2 & 5.4 & 12.1 & -3.7 & -0.4 & 1.8 & 5.0 & 7.0 & 7.4 & \\
\hline Growth of imports of goods and services (U.S. dollar terms, in percent) 6/ & 44.7 & 29.6 & 6.3 & 28.7 & -13.7 & -2.1 & 5.9 & 5.0 & 5.4 & 6.4 & \\
\hline Current account balance, excluding interest payments & -0.5 & -2.9 & -3.8 & -7.1 & -4.5 & -3.2 & -3.7 & -3.6 & -3.3 & -3.1 & \\
\hline Net non-debt-creating capital inflows & 1.5 & 3.1 & 5.2 & 3.3 & 1.9 & 1.8 & 2.1 & 2.4 & 2.6 & 2.8 & \\
\hline
\end{tabular}

Sources: Pakistani authorities; and Fund staff estimates and projections.

1/ Derived as $[r-g-\rho(1+g)+e a(1+r)] /(1+g+\rho+g \rho)$ times previous period debt stock, with $r=$ nominal effective interest rate on external debt; $\rho=$ change in domestic GDP deflator in U.S. dollar terms, $\mathrm{g}=$ real GDP growth rate, $\mathrm{e}=$ nominal appreciation (increase in dollar value of domestic currency), and $\mathrm{a}=$ share of domestic-currency denominated debt in total external debt.

$2 /$ The contribution from price and exchange rate changes is defined as $[-\rho(1+g)+e a(1+r)] /(1+g+\rho+g \rho)$ times previous period debt stock. rho increases with an appreciating domestic currency $(\mathrm{e}>0)$ and rising inflation (based on GDP deflator).

3/ For projection, line includes the impact of price and exchange rate changes.

4/ Defined as current account deficit, plus amortization on medium- and long-term debt, plus short-term debt at end of previous period.

5/ The key variables include real GDP growth; nominal interest rate; dollar deflator growth; and both non-interest current account and non-debt inflows in percent of GDP.

6/ Exports and imports of goods and services

7/ Long-run, constant balance that stabilizes the debt ratio assuming that key variables (real GDP growth, nominal interest rate, dollar deflator growth, and non-debt inflows in percent of GDP)

remain at their levels of the last projection year. 

Figure 2. Pakistan: Public Debt Sustainability: Bound Tests $1 /$
(Public debt in percent of GDP)
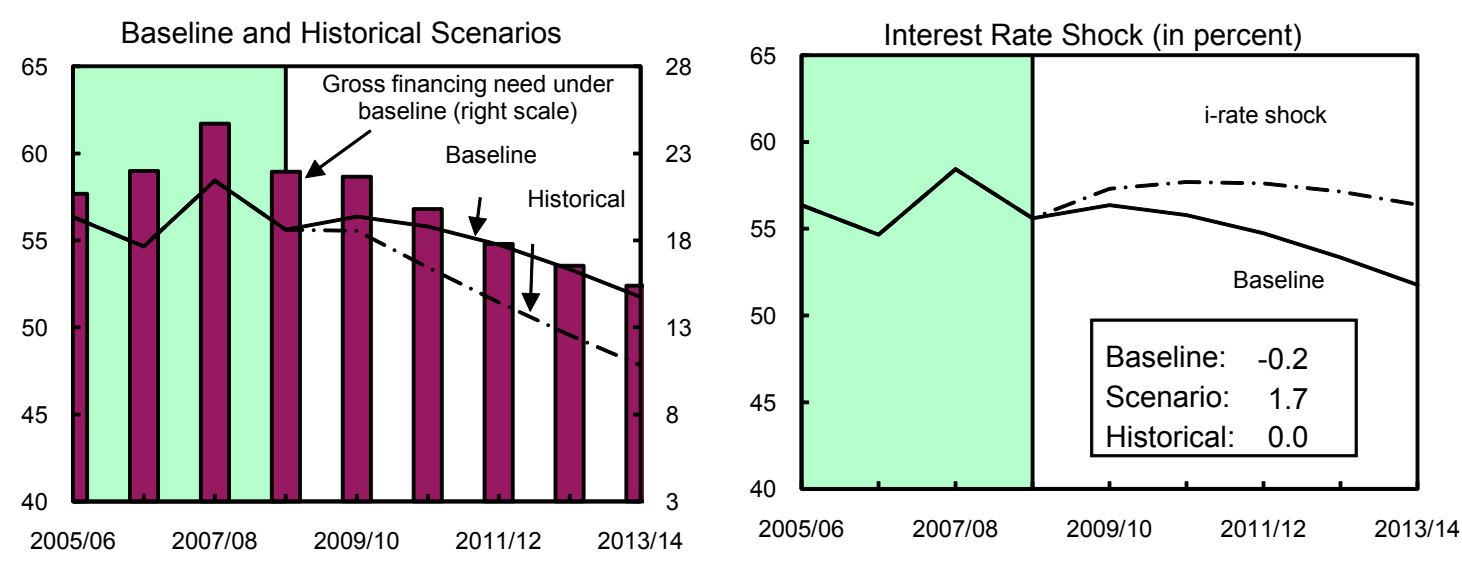

Growth Shock (in percent per year)

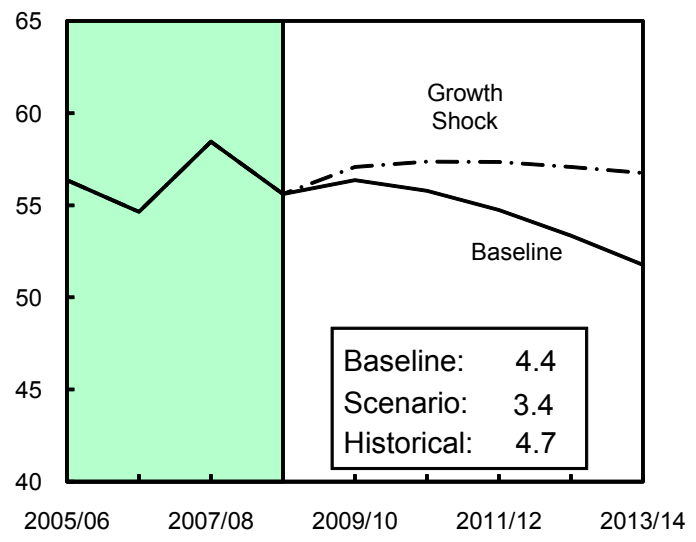

Primary Balance Shock (in percent of GDP) and No policy Change Scenario (constant primary balance)

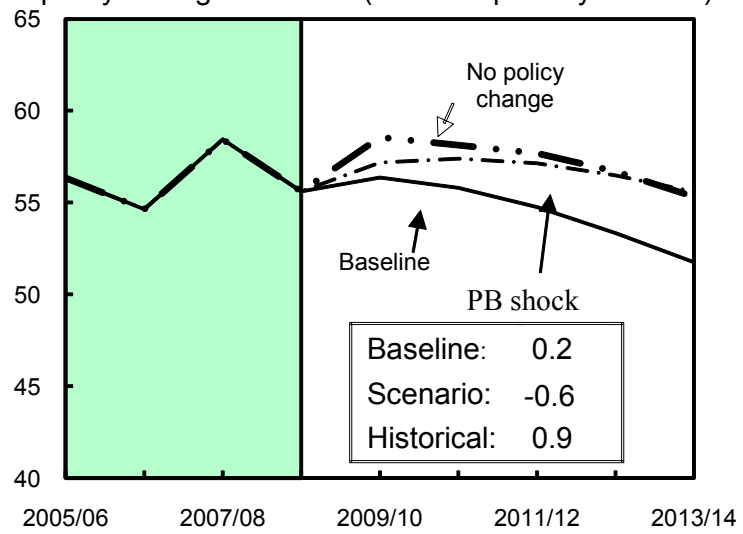

Combined Shock 2/

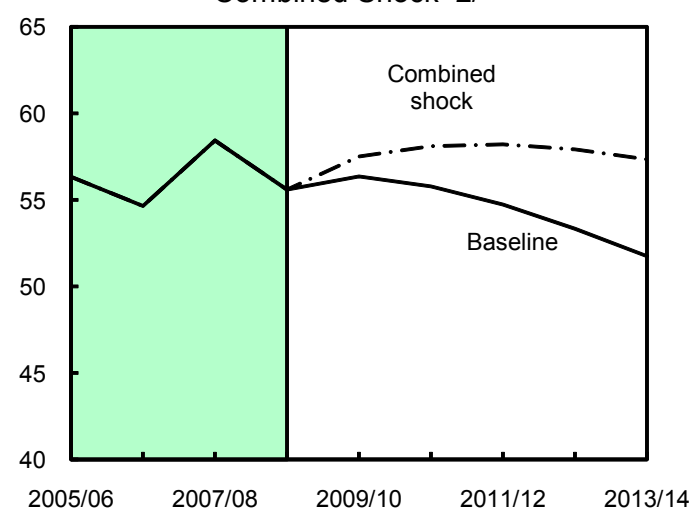

Real Depreciation and Contingent Liabilities Shocks

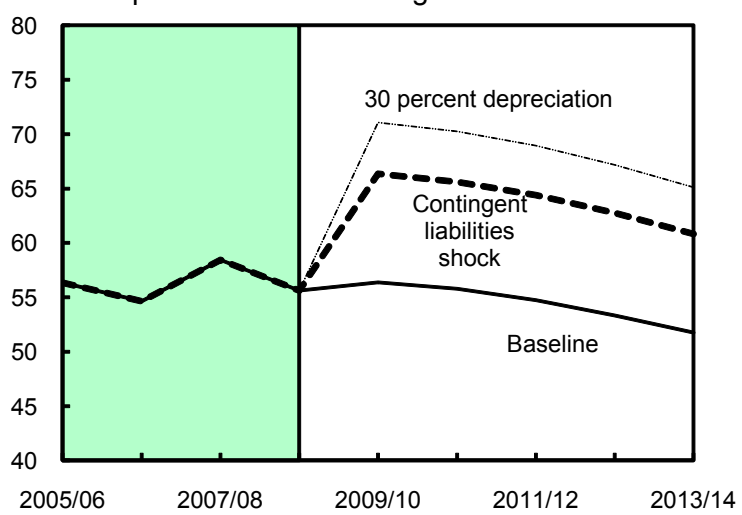

Source: Fund staff estimates and projections.

1/ Shaded areas represent actual data. Individual shocks are permanent one-half standard deviation shocks. Figures in the boxes represent average projections for the respective variables in the baseline and scenario being presented. Ten-year historical average for the variable is also shown.

2/ Permanent $1 / 4$ standard deviation shocks applied to real interest rate, growth rate, and primary balance.

3/ One-time real depreciation of 30 percent and 10 percent of GDP shock to contingent liabilities occur in 2009/10, with real depreciation defined as nominal depreciation (measured by percentage fall in dollar value of local currency) minus domestic inflation (based on GDP deflator). 
Table 2. Pakistan: Public Sector Debt Sustainability Framework, 2004/05-2013/14

(In percent of GDP, unless otherwise indicated)

\begin{tabular}{|c|c|c|c|c|c|c|c|c|c|c|c|}
\hline \multirow[b]{2}{*}{$\begin{array}{l}\text { Baseline: Public sector debt 1/ } \\
\text { Of which: Foreign-currency denominated }\end{array}$} & \multicolumn{4}{|c|}{$\frac{\text { Actual }}{2004 / 052005 / 062006 / 072007 / 08}$} & \multicolumn{6}{|c|}{$\begin{array}{c}\text { Projections } \\
2008 / 092009 / 102010 / 112011 / 12 \quad 2012 / 13 \quad 2013 / 14\end{array}$} & \multirow{2}{*}{$\begin{array}{c}\begin{array}{r}\text { Debt-stabilizing } \\
\text { primary balance 9/ }\end{array} \\
-1.3\end{array}$} \\
\hline & $\begin{array}{l}62.0 \\
28.9\end{array}$ & $\begin{array}{l}56.4 \\
25.9\end{array}$ & $\begin{array}{l}54.6 \\
24.7\end{array}$ & $\begin{array}{l}58.4 \\
26.7\end{array}$ & $\begin{array}{l}55.6 \\
26.2\end{array}$ & $\begin{array}{l}56.4 \\
25.4\end{array}$ & $\begin{array}{l}55.8 \\
26.3\end{array}$ & $\begin{array}{l}54.7 \\
27.4\end{array}$ & $\begin{array}{l}53.3 \\
27.9\end{array}$ & $\begin{array}{l}51.8 \\
28.0\end{array}$ & \\
\hline Change in public sector debt & -6.3 & -5.6 & -1.7 & 3.8 & -2.8 & 0.8 & -0.6 & -1.0 & -1.4 & -1.6 & \\
\hline Identified debt-creating flows $(4+7+12)$ & -4.3 & -5.0 & -2.6 & 1.1 & -2.4 & -2.2 & -1.6 & -2.1 & -2.5 & -2.7 & \\
\hline Primary deficit & -0.2 & 0.6 & -0.2 & 2.5 & 0.1 & -0.1 & 0.0 & -0.4 & -0.3 & -0.2 & \\
\hline Revenue and grants & 14.1 & 14.7 & 15.3 & 14.9 & 14.3 & 15.2 & 15.5 & 16.1 & 16.4 & 16.7 & \\
\hline Primary (noninterest) expenditure & 14.0 & 15.3 & 15.1 & 17.4 & 14.5 & 15.1 & 15.5 & 15.7 & 16.1 & 16.5 & \\
\hline Automatic debt dynamics 21 & -5.0 & -5.8 & -2.4 & -1.1 & -3.5 & -2.6 & -2.0 & -2.0 & -2.5 & -2.9 & \\
\hline Contribution from interest rate/growth differential $3 /$ & -5.8 & -6.0 & -2.6 & -3.8 & -7.7 & -2.6 & -2.0 & -2.0 & -2.5 & -2.9 & \\
\hline Of which: Contribution from real interest rate & -1.2 & -2.8 & 0.2 & -2.9 & -6.8 & -1.1 & 0.1 & 0.2 & -0.1 & -0.3 & \\
\hline Of which: Contribution from real GDP growth & -4.5 & -3.2 & -2.8 & -0.9 & -0.9 & -1.5 & -2.0 & -2.3 & -2.5 & -2.6 & \\
\hline Contribution from exchange rate depreciation $4 /$ & 0.8 & 0.2 & 0.1 & 2.7 & 4.2 & & & & & & \\
\hline Other identified debt-creating flows & 0.9 & 0.2 & 0.1 & -0.3 & 1.0 & 0.4 & 0.3 & 0.3 & 0.3 & 0.4 & \\
\hline Privatization receipts (negative) & -0.4 & -1.3 & -0.8 & 0.0 & 0.0 & -0.2 & -0.2 & -0.2 & -0.1 & -0.1 & \\
\hline Recognition of implicit or contingent liabilities & 0.0 & 0.0 & 0.0 & 0.0 & 0.0 & 0.0 & 0.0 & 0.0 & 0.0 & 0.0 & \\
\hline Build-up of bank deposits & 1.3 & 1.5 & 0.9 & -0.3 & 1.0 & 0.6 & 0.5 & 0.5 & 0.5 & 0.5 & \\
\hline Residual, including other asset changes (2-3) 5 / & -2.0 & -0.6 & 0.9 & 2.7 & -0.5 & 3.0 & 1.0 & 1.1 & 1.1 & 1.1 & \\
\hline Public sector debt-to-revenue ratio $1 /$ & 438.2 & 383.3 & 357.1 & 392.8 & 388.0 & 370.2 & 359.7 & 340.7 & 325.7 & 310.0 & \\
\hline Gross financing need 6/ & 19.0 & 20.7 & 22.0 & 24.7 & 22.0 & 21.7 & 19.8 & 17.8 & 16.6 & 15.4 & \\
\hline In billions of U.S. dollars & 20.8 & 26.4 & 31.5 & 40.7 & 36.6 & 38.3 & 37.2 & 35.4 & 35.0 & 34.9 & \\
\hline Scenario with key variables at their historical averages $7 /$ & $\ldots$ & $\ldots$ & $\ldots$ & $\ldots$ & 55.6 & 55.6 & 53.5 & 51.4 & 49.5 & 47.8 & -0.7 \\
\hline Scenario with no policy change (constant primary balance) in 2009/10-2013/14 & $\ldots$ & $\ldots$ & $\ldots$ & $\ldots$ & 55.6 & 58.5 & 58.1 & 57.7 & 56.7 & 55.3 & -1.4 \\
\hline \multicolumn{12}{|l|}{ Key Macroeconomic and Fiscal Assumptions Underlying Baseline } \\
\hline Real GDP growth at market prices (in percent) & 7.7 & 6.1 & 5.6 & 2.0 & 2.0 & 3.0 & 4.0 & 4.5 & 5.0 & 5.5 & \\
\hline Average nominal interest rate on public debt (in percent) $8 /$ & 5.5 & 5.9 & 8.6 & 10.3 & 10.6 & 9.1 & 7.4 & 6.7 & 6.2 & 5.7 & \\
\hline Average real interest rate (nominal rate minus change in GDP deflator, in percent) & -1.6 & -4.6 & 0.9 & -5.9 & -14.3 & -1.9 & 0.4 & 0.7 & 0.2 & -0.3 & \\
\hline Nominal appreciation (increase in U.S. dollar value of local currency, in percent) & -2.5 & -0.9 & -0.5 & -11.4 & & & & & & & \\
\hline Inflation rate (GDP deflator, in percent) & 7.0 & 10.5 & 7.7 & 16.2 & 24.9 & 11.0 & 7.0 & 6.0 & 6.0 & 6.0 & \\
\hline Growth of real primary spending (deflated by GDP deflator, in percent) & 16.5 & 16.2 & 4.2 & 17.8 & -15.2 & 7.9 & 6.7 & 5.4 & 7.7 & 8.5 & \\
\hline Primary deficit & -0.2 & 0.6 & -0.2 & 2.5 & 0.1 & -0.1 & 0.0 & -0.4 & -0.3 & -0.2 & \\
\hline
\end{tabular}

Sources: Pakistani authorities; and Fund staff estimates and projections.

$1 /$ General government gross debt; excludes obligations to the IMF, external military debt, commercial debt, short-term loans, and debt from public enterprises.

2/ Derived as $[(r-p(1+g)-g+a e(1+r)] /(1+g+p+g p))$ times previous period debt ratio, with $r=$ interest rate; $p=$ growth rate of GDP deflator; $g=$ real GDP growth rate; $a=$ share of foreign-currency denominated debt; and $e=$ nominal exchange rate depreciation (measured by increase $i \mathrm{rl}$ local currency value of $U$.S. dollar).

$4 /$ The realing -9 .

作 7/ The

.

9/ Assumes that key variables (real GDP growth, real interest rate, and other identified debt-creating flows) remain at the level of the last projection year 


\section{ATTACHMENT I. LETTER OF INTENT}

December 11, 2009

Mr. Dominique Strauss-Kahn

Managing Director

International Monetary Fund

Washington, D.C. 20431

Dear Mr. Strauss-Kahn:

The Pakistani authorities held discussions with Fund staff for the third review under the Stand-By Arrangement (SBA). Based on these discussions, we have updated our economic program for 2009/10, as reflected in the attached Third Supplementary Memorandum of Economic and Financial Policies (SMEFP). In support of our policies, the government requests that the Executive Board of the Fund complete the third review, and approve the fourth purchase under the SBA of SDR 766.7 million.

Our stabilization program has advanced, notwithstanding challenging circumstances. Military campaigns in Swat and South Waziristan are adding to budget outlays for security, and depressed global economic activity and higher oil prices have affected Pakistan's domestic economy, trade, and external financing. We believe that the policies set out in the attached SMEFP are adequate to further consolidate macroeconomic stability and advance our ambitious reform agenda. However, our authorities stand ready to take any additional measures deemed appropriate for this purpose, and will consult with the Fund on the adoption of these measures in accordance with Fund policies on such consultations. We will provide the Fund with the information it may request to monitor progress in implementing its economic and financial policies.

We request a waiver of nonobservance for the end-September 2009 quantitative performance criterion on the fiscal deficit. The original deficit target was missed by about 0.2 percent of GDP, but the target was adjusted downward for the shortfall in external grants, which increased the overrun to 0.3 percent of GDP. The fiscal overrun was due to a delay in receipt of a large service payment, an extension of the period for advance income tax payments to October 15, 2009, an advance salary payment to federal and provincial employees in September due to the Eid holiday, and higher security spending. We also request: (i) a modification of the end-December 2009 performance criterion on the fiscal deficit to increase it by Rs. 15 billion ( 0.1 percent of GDP) to allow space for urgent military spending and avoid undue cuts in other priority spending; (ii) a modification of the end-December 2009 performance criterion on the net foreign assets and net domestic assets of the State Bank of Pakistan to reflect revised projections; and (iii) establishment of performance criteria for endMarch 2010 and end-June 2010. 
We consent to the publication of this letter, the attached SMEFP, the revised Technical Memorandum of Understanding, and the related staff report.

Sincerely yours,

$/ / \mathrm{s} / /$

Shaukat Tarin

Minister of Finance, Revenue, Economic Affairs and Statistics

\author{
$/ / \mathrm{S} / /$ \\ Syed Salim Raza \\ Governor \\ State Bank of Pakistan
}

Attachments:

Third Supplementary Memorandum of Economic and Financial Policies Technical Memorandum of Understanding 


\title{
AtTaChment II. Pakistan: Third SupPlementary Memorandum ON ECONOMIC AND FinANCIAL POLICIES FOR 2009/10
}

\author{
December 11, 2009
}

\section{INTRODUCTION}

1. This memorandum supplements our Memorandum on Economic and Financial Policies (MEFP) for 2008/09-09/10 of November 20, 2008 and the first and second supplements thereto dated March 16 and July 29, 2009, respectively. It describes policy implementation to date and lays out additional policies agreed in the context of the third review under the Stand-By Arrangement (SBA).

\section{RECENT ECONOMIC DEVELOPMENTS}

\section{Our stabilization program has advanced, notwithstanding challenging}

circumstances. Military campaigns in Swat and South Waziristan are adding to budget outlays for security. The South Warizistan campaign was not anticipated at the time of the budget formulation. Headline inflation declined from 25 percent in October 2008 to 8.9 percent in October 2009, the rupee has remained stable at around Rs. 83-84 per U.S. dollar in recent months, gross foreign exchange reserves reached $\$ 10.1$ billion as of end-November, 2009, and market demand for Treasury bills has remained stable. All quantitative performance criteria for end-September 2009 were observed (Table 1), except for the budget deficit target, which was exceeded by 0.3 percent of annual GDP. The original deficit target was missed by about 0.2 percent of GDP, but the target was adjusted downward for the shortfall in external grants, which increased the overrun to 0.3 percent of GDP. The structural reforms are proceeding and we are pressing ahead and remain fully committed to implementing our reform agenda, although there have been delays in implementation.

3. Despite electricity shortages, external factors, and security concerns, there are signs of revival in economic activity. The deterioration in domestic security conditions, electricity shortages, and weak external demand for Pakistan exports have depressed output. However, recently there have been signs of a revival in economic activity, with a pick-up in output in a few sectors and increased economic confidence. Financial markets also point to an improvement. The stock market index has risen, in part driven by private capital inflows in recent months, the sovereign credit rating has been raised, and the spreads on Pakistan sovereign bonds have declined.

\section{The slippage in fiscal performance in the first quarter of 2009/10 was due} mostly to temporary factors. Four principal factors (totaling 0.8 percent of GDP) accounted for this slippage, and we acted to offset most of their impact on the budget. These factors were: (i) a delay in a large service payment; (ii) an extension of the period for advance income tax payments to October 15, 2009; (iii) an advanced salary payment to federal and provincial employees in September due to the Eid holiday; and (iv) higher security spending. We were able to limit the fiscal slippage to 0.3 percent of GDP through expenditure control. 
5. Monetary policy has continued to focus on price stability. After reducing the discount rate by 100 bps percent to 13 percent in August, the SBP cut rates by 50 bps on November 25. This modest cut took into account fiscal uncertainties as well as expected inflationary pressures from higher energy and food prices. On August 17, the SBP successfully introduced an interest rate corridor to enhance monetary management and signaling of its policy stance.

\section{External support-crucial for ensuring success of stabilization efforts-is}

lagging. The 2009/10 budget was prepared keeping in mind external inflows pledged by donors at Tokyo in April 2009. Although donors have made firm commitments, it is vital that these pledges materialize in a timely manner during the current fiscal year.

\section{POLICIES FOR THE REMAINDER OF 2009/10}

\section{We will continue our efforts to consolidate macroeconomic stability, reduce} poverty, and lay the basis for high and sustained growth. A principal challenge for this year will be to consolidate macroeconomic stability in the context of an increasingly difficult security situation. We will also continue to provide social safety nets for the poor. At the same time, we will press ahead with the implementation of tax reform, including a broad-based VAT.

\section{Our program for the remainder of 2009/10 is based on the following} projections. Real GDP is expected to grow by 3 percent and while there are external and domestic risks, we believe this growth is attainable. The projection for consumer price inflation through end-June 2010 is 11 percent (year-on-year), and the external current account deficit is expected to decline to 4.2 percent of GDP. The current account position is stronger than forecasted earlier, despite somewhat higher import costs for oil, due to a significant improvement in the expected flow of remittances owing to the Pakistan Remittance Initiative.

9. Early disbursement of the donor support pledged in Tokyo is needed to keep our stabilization program on track. This donor support will protect budgeted social and growth-oriented development spending, and help finance support for internally displaced persons (IDPs). Although little support has been received since July, firm commitments have been received and timely disbursements will be needed in order to protect key social and development spending. We will continue to work with donors to facilitate prompt disbursement of Tokyo and other pledges.

\section{A. Fiscal Policy}

10. The fiscal deficit target for 2009/10 remains unchanged at 4.9 percent of GDP (excluding grants). The government is resolved to stay the course with its stabilization program. Mounting fiscal pressures due to the electricity sector and higher security outlays will be offset:

- As noted, the budget overrun in the first quarter of the fiscal year will be reversed, mostly in the second quarter. Higher income tax collections in October are encouraging. The date of the service payment is still under discussion but the 
advance payment of salaries in September has already been compensated by lower payments in October.

- $\quad$ We intend to achieve the 2009/10 target for Federal Board of Revenue (FBR) collections of Rs. 1,396 billion. A higher international oil price as well as the planned increase in electricity tariffs should boost customs and sales tax revenue, offsetting weaker excise and direct tax receipts. In addition, in the third quarter of 2009/10, we will increase the rate of the existing special excise duty, and - if needed-reduce the capital value tax exemptions.

- $\quad$ Electricity tariff differential subsidies for the year will be contained at Rs. 55 billion. We will take measures to make up for the delay in implementing the monthly fuel price adjustments, and a possible shortage of gas (see below) through the quarterly tariff adjustment in mid-December.

- $\quad$ Although there are risks to the fiscal targets, promised grant financing will accommodate the additional security spending in South Waziristan, as well as IDPs. This should greatly limit the need to cut development spending. To this effect, we are seeking from donors 0.3 percent of GDP in grants to help cover expenditures on IDPs. Should the grants for this purpose fall short of this amount, the deficit (excluding grants) would be reduced correspondingly; however, the expenditures on IDPs as well as on the social safety net and education will be protected in the budget.

\section{Financial controls on borrowing by provinces have been strengthened.}

Borrowing by provinces is limited to Ways and Means advances equivalent to six weeks of provincial wages and salaries totaling Rs. 47 billion. Agreements on these limits have been signed by the SBP and the provinces and are being enforced rigorously. Agreements have also been reached between the SBP and Punjab and Baluchistan to convert their outstanding overdrafts of Rs. 50.9 billion and Rs. 8.8 billion, respectively, into four-year loans with interest equal to the 6-month T-bill rate, to be repaid in monthly installments. The government provided a guarantee to the SBP for these loans on November 23, 2009. For the other two provinces, no long-term loans were needed. Further, there are regular monthly meetings between the Federal Ministry of Finance and Provincial Finance Departments to monitor the provinces' financial positions and ensure that they are consistent with the fiscal targets.

12. We are making progress on the introduction of a broad-based VAT by July 1, 2010 to meet the government's medium-term revenue needs. It is estimated that the additional revenue generated could reach 3 percent of GDP per annum over the medium term. Much of this will be generated by removing domestic zero rating and reducing exemptions to expand the tax base. We have started consulting stakeholders on the VAT with a seminar in September, with the participation of members of the federal and provincial governments, and supported by the IMF and the World Bank. This was followed by a drafting workshop on VAT legislation in early November. We have adopted a time-bound action plan for the introduction of the VAT. The draft law will 
be finalized in early December and submitted to parliament by end-December 2009 (structural benchmark). We also plan to submit legislation to harmonize the existing tax laws with the VAT law by end-April, 2010. We will prepare the needed regulations for the full implementation of the VAT by July 1, 2010. Full implementation of the VAT by July 1, 2010 is a key objective and a structural benchmark. We expect continuation of IMF and World Bank assistance on policy aspects for introducing the VAT.

\section{Reforming tax administration has been subjected to legal challenges, but we} remain committed to our objective of implementing a modern and integrated tax administration. A key objective, the establishment of a functionally structured tax administration within the FBR - that integrates responsibilities for domestic taxes, including the sales, income, and excise taxes, in one occupational group - has been achieved. A functionally structured system will increase the effectiveness of tax administration and facilitate VAT introduction. The following actions to improve tax administration have been taken or will be adopted:

- $\quad$ On September 12, the government, under the existing Civil Servants Act 1973, has established an Inland Revenue Service as a new occupational group within the FBR (structural benchmark).

- An ordinance to harmonize the income tax and the existing GST law was issued on October 28. This ordinance will be amended given the new VAT law and renewed as needed, and submitted to parliament by end-April 2010.

- To institute the organizational changes to implement the new inland revenue tax administration a notification to revise the structures of Regional Taxpayer Offices (RTOs) and Large Taxpayer Units (LTUs) (structural benchmark) was issued on November 11.

- The option for retailers with a turnover over Rs. 10 million to choose the presumptive tax regime will be eliminated in the new VAT law.

- $\quad$ The FBR has been implementing its action plan on tax administration reform. We are focusing on the following:

- To improve sales tax and corporate income tax compliance we will reduce the number of under-reporting taxpayers and nonfilers. We have identified 13,000 under-reporting taxpayers and about one million taxpayers (about 40 percent of the taxpayers with tax identification number) who do not file tax returns. Our efforts will focus on underreporting taxpayers. A letter will be sent to these under-reporting taxpayers by end-December 2009 to invite them to file tax returns and settle their prospective unpaid taxes. Letters to identified non-filers at the rate of 100,000 per month will begin by January 1, 2010. This will be preceded by a publicity campaign starting in November. In cases where this voluntary approach is not successful, we will institute legal claims procedures for noncompliant and nonfiling taxpayers. 
- Tax audit and enforcement plans have been formulated and approved by the FBR Board. We have identified 850 companies (15 percent of large taxpayers) for audit by either the FBR or external auditors. We plan to complete most of these audits by end-March. Importantly, in the future, we plan to conduct the audits on a regular basis. To that effect, we will strengthen the auditing capacity of the FBR.

- We are committed to strengthening the system of tax refunds. The government has approved a strengthened and improved electronic payment and refund system. We have held technical meetings with banks to establish an electronic filing, payment and refund system and have set up a technical committee to finalize all procedures. The expedited sales tax refund system is being piloted in Islamabad, Karachi and Lahore. We expect the pilots to be completed and evaluated by end-December, 2009. We will then rollout this system nationwide, and an expedited sales tax refund system - to ensure direct input of refund requests and prompt processing and confirmation of refunds - will be operational in all RTOs and LTUs by end-March, 2010 (structural benchmark) and ready for the introduction of the VAT.

14. We recently introduced subsidies for companies that borrow under the SBP's Export Financing Scheme and Long-Term Financing Facility. This change has been made to improve transparency by moving quasi-fiscal subsidies (previously provided by the SBP) to the budget outlays. The impact of these subsidies on budget expenditures are expected to be offset by higher profit transfers from the SBP.

15. We remain committed to strengthening the social safety net. We have completed the pilot phase of the new strengthened targeting mechanism (scorecard) for the Benazir Income Support Program (BISP), and are starting the preparations of the nationwide rollout of the new targeting mechanism. The nationwide rollout has been delayed by unforeseen issues as well as administrative capacity constraints. We expect this nationwide rollout to be completed by end 2010, after which all recipients of BISP benefits will be selected using the scorecard system. Meanwhile, we are using the existing targeting mechanism. To offset its weaknesses while the scorecard targeting system is rolled out, we will establish local offices at the Tehsil level, provide mechanisms for grievances and complaints, and develop and test alternative payment methods. Further, in September 2009, we introduced the Wasilla-ul-Haq credit program as one of the potential graduation strategies for BISP recipients to exit poverty. We plan to revisit the design of this program and ensure that BISP has the appropriate institutional capacity for the nationwide rollout of the poverty scorecard before any decision is taken on the expansion of graduation programs.

16. We will continue to improve our budget expenditure management framework. To this end, we plan to invigorate and deepen reforms under the Pakistan Improvement of Financial Reporting and Auditing Project (PIFRA) that will strengthen budget data reporting and set the stage for commitment control. The first step will be an independent 
review of PIFRA with Fund technical assistance to be completed by end-June 2010 (structural benchmark). The review will aim at improving reporting, laying the basis for commitment control, and assess in which institution PIFRA will be located. In addition, we plan to further strengthen the medium-term budget framework that was introduced with the 2009/10 budget. The 2010/11 budget framework will link current and development spending under the MTBF. It will improve expenditure planning and predictability of resource availability and the effectiveness of the public sector development program by increasing the predictability and quality of expenditure.

\section{The transition to a Treasury Single Account (Federal Consolidated Fund) will} be completed gradually. The government requires - for entities under the assignment account framework - that all disbursements pass through a zero-balance assignment account, and has taken measures to ensure that leakage of cash into commercial bank accounts does not occur. With World Bank technical assistance, a survey of the remaining balances with commercial banks of the largest spending agencies of the federal government was initiated in November 2009 to verify their compliance with the policy on the use of assignment accounts. Based on the results of this survey, the funds remaining in commercial banks of these entities will be transferred to the Federal Consolidated Fund by end-June 2010, while ensuring that this transfer does not affect banking sector liquidity negatively. Further, all provincial governments will complete the transition of their accounts to the assignment account mechanism by end-June, 2010 (structural benchmark).

\section{We are implementing the plan developed with World Bank and Asian} Development Bank staffs to eliminate electricity tariff differential subsidies by August 2010. The first adjustment of electricity tariffs was partly implemented on October 1, 2009, and the remainder of this increase will be implemented in December. Tariffs will be adjusted further in stages to ensure that they reach cost recovery in August 2010, and we will ensure that the tariff differential subsidy is limited to Rs. 55 billion in 2009/10. The implementation of the automatic monthly surcharge for changes in fuel prices and power purchase costs was delayed from end-August and implemented on October 30. Electricity tariffs will be adjusted monthly (by middle of each month) to reflect the changes in fuel prices and power purchase costs. The first quarterly determination of overall electricity tariffs by NEPRA was completed on December 10 and will be notified by December 20, 2009 , and we will continue with regular quarterly determinations and notifications in the future. The ordinance amending the NEPRA Act issued in July, which provides for the monthly surcharge and quarterly determination of overall electricity tariffs, has been renewed to ensure the amendments under the NEPRA Act remain in force.

\section{Progress is being made to resolve the issue of circular debt in the electricity}

sector. The Holding Company under the Ministry of Water and Power has been established and a Board of Directors has been appointed. The Holding Company issued Rs. 85 billion of Term Finance Certificates (TFCs) on September 30, with government guarantee. TFCs are now traded at the stock exchange. The transfer of the bulk of the remaining stock of circular debt of Rs. 216 billion is expected to take place by endMarch, 2010; the government has serviced this debt since July 1, 2009. An international 
firm has audited the debt from 2008/09 and earlier years, which is slated to be assumed by the Holding Company. Further, to encourage an improved financial performance in this sector, we have instituted regular monthly meetings with stakeholders to ensure subsidy needs remain at or below targeted levels.

20. We are also monitoring closely the issuance of government guarantees. We have published the flow of new guarantees issued on a monthly basis on the Ministry of Finance website, and will supplement, by end-March 2010, this information with the total outstanding stock of government guarantees that will be published monthly.

21. We are preparing a debt management strategy. This medium-term strategy will improve coordination of fiscal and monetary policies, support capital market development, mobilize savings, and seek to develop nonbank financing. The strategy will set criteria to determine the currency composition, interest rate basis (fixed or floating) and maturity structure of public and publicly guaranteed (domestic and foreign) debt. It will also set out mechanisms to strengthen coordination between the Ministry of Finance, the SBP, and the Central Directorate for National Saving in the near term, as well as medium-term initiatives to streamline the institutional arrangements for debt management operations.

\section{B. Monetary and Exchange Rate Policies}

\section{The SBP's primary monetary policy objective is to reduce inflation while} continuing to strengthen the international reserve position. Decisions to adjust the SBP policy rate will take into account the recent improvement in inflation while remaining cognizant of supply-side price shocks and fiscal uncertainties. The SBP's liquidity management will also seek to ensure the smooth functioning of the domestic market.

\section{The introduction of the SBP's new monetary policy framework-a corridor of} 300 basis points for the over-night money-market repo rate-was smooth. The objective of the new framework is to reduce the volatility of short-term interest rates and improve the transmission of monetary policy signals. Thus far, there has been limited volatility in the weighted average overnight repo rate. To enhance the functioning of the corridor, the SBP may consider measures related to reserve requirements and the associated maintenance period. In addition, the SBP and the government will continue to enhance their coordination and communication to strengthen the liquidity-forecasting framework.

\section{The SBP will continue to pursue a flexible exchange rate policy to strengthen} the external position and maintain competitiveness. In this context, interventions in the foreign exchange market will be largely aimed at achieving the program's NFA targets. The SBP has phased out the provision of foreign exchange for oil imports and notified the market that it will cease to provide foreign exchange for imports of crude oil effective December 14, 2009.

\section{The SBP will rationalize its refinancing schemes and eliminate the subsidies} resulting from below-market interest rates. As a first step, the interest rate for the Export Financing Scheme (EFS) and the Long-Term Financing Facility (LTFF) has been 
increased on November 1 to 8 percent and 9.2-10.25 percent (depending on tenor), respectively. Further steps will be taken to bring the rate to the level of the benchmarks of weighted average yield on six-month T-bills and yields of the same tenor for Pakistani Investment Bonds, respectively. We will continue with gradual increases to reach levels two percentage points below the benchmarks by end-September 2010, and rates will equal their benchmarks by end-September 2011.

\section{Financial Sector}

\section{We remain committed to ensuring financial stability and will continue to} monitor financial sector soundness closely. Financial sector soundness indicators through end-September have deteriorated slightly compared to end-March and end-June. Gross nonperforming loans (NPLs) increased by nearly 6 percent in the first quarter of 2009/10, and the gross NPL-to-loans ratio rose to 12.4 percent. Nevertheless, generally banks remained well-capitalized and profitable, although bank profitability was lower than in the same period of 2008/09. A few banks with capital deficiencies have either capital injection plans already in place or are in active dialogue with the central bank to decide on the strategy to meet capital adequacy targets (end-December 2009). A continued shift of bank assets from lending to the private sector credit to investment in government paper and lending to public sector enterprises improved the average riskadjusted capital adequacy ratio (CAR) of the banking sector.

27. The SBP introduced regulatory changes to facilitate restructuring of NPLs by commercial banks and development financial institutions. Regulatory provisions have been amended to allow banks to increase the benefit of forced sale value of collateral from 30 percent to 40 percent for calculating provisioning requirements. Moreover, additional interim instructions have been introduced on classification/provisioning requirements with a view to facilitating the rescheduling or restructuring of NPLs. At the same time, the SBP took adequate measures to limit moral hazard from possible evergreening of NPLs. These include monthly reporting on the rescheduled NPLs and limiting the benefit from a change in provisions only to increase general reserves and not for payment of cash or dividends to shareholders. The SBP also encouraged the banks to coordinate their actions for the recovery proceedings against defaulting groups/companies that have substantial exposure from a number of banks.

\section{Amendments to the Banking Companies Ordinance have been submitted to}

Parliament on October 2, 2009 (structural benchmark). These amendments will enhance the effectiveness of SBP enforcement in the area of banking supervision. The amendments were approved on November 3, 2009 by the parliament's standing committee on Finance and Revenue and submitted for plenary discussion and approval.

29. Amendments to the SBP law to enhance the SBP's operational independence will be submitted to Parliament by December 20, 2009. Ahead of adoption of the new law, the SBP has already announced the composition of the nine-member Monetary Policy Committee of the SBP Board, which has started its work in November 2009. 
30. Consultations on the new bankruptcy law (Corporate Rehabilitation Act) have reached a final stage. The new law will help both the corporate and financial sectors by strengthening the legal basis for rehabilitation of viable but struggling corporate borrowers and speeding up the process of liquidation of unviable entities. Submission of the draft law to parliament has been delayed in order to allay concerns raised by financial institutions regarding creditor protection. The revised law was submitted to the Minister of Finance on November 16, 2009 and submission to parliament is expected by endMarch, 2010.

\section{Program Risks ANd Monitoring}

31. The risks to the program remain. Delays in mobilizing disbursements of donor support would limit the scope for higher expenditure and add to pressures on the budget and domestic financing. Other risks include deterioration in the outlook for Pakistan's trading partners, which could result in lower demand for exports, and smaller private capital flows, which to some extent is being mitigated by the well-structured Pakistan Remittance Initiative. Also, adverse security developments add to budget pressures. Moreover, if economic activity weakens, the risks to the financial sector may increase.

32. The program will remain subject to quarterly program reviews and quarterly performance criteria for 2009/10, as specified in (Table 1). We request that the endDecember 2009 performance criterion on the fiscal deficit be revised to allow space for urgent security spending and avoid undue cuts in other priority spending and the endDecember performance criteria on the net foreign assets and net domestic assets of the SBP be revised to reflect updated projections. Quarterly performance criteria will be set for end-March 2010 and end-June 2010. The attached Technical Memorandum of Understanding (TMU) replaces the TMU of July 29, 2009.

\section{The following measures will be prior actions for Board consideration of the} staff report for the third review: (i) initiate taxpayer audits among the 850 companies identified for audit by either the FBR or external auditors and (ii) government approval of an electronic payment and tax refund system.

\section{We propose to introduce the following new structural benchmarks.}

(i) implement expedited sales tax refund system - to ensure direct input of refund requests and prompt processing and confirmation of refunds - in all RTOs and LTUs by endMarch, 2010; (ii) introduction of the VAT by July 1, 2010; and (iii) complete an independent review of PIFRA (by end-June 2010) to improve reporting, lay the basis for commitment control, and assess in which institution PIFRA will be located.

\section{Program Financing}

35. The program remains fully financed but disbursement of the assistance pledged in Tokyo is crucial. 
Table 1. Pakistan: Quantitative Criteria, December 2008-June 2010

\begin{tabular}{|c|c|c|c|c|c|c|c|}
\hline & $\begin{array}{l}\text { Outst. Stock } \\
\text { end-Sept. } \\
2008\end{array}$ & $\begin{array}{l}\text { end-Mar. } \\
2009\end{array}$ & $\begin{array}{l}\text { end-Jun. } \\
2009\end{array}$ & $\begin{array}{l}\text { end-Sept. } \\
2009\end{array}$ & $\begin{array}{l}\text { Prog. } 2 / \\
\text { end-Dec. } \\
2009\end{array}$ & $\begin{array}{l}\text { Prog. } 2 / \\
\text { end-Mar. } \\
2010\end{array}$ & $\begin{array}{l}\text { Prog. } 2 / \\
\text { end-Jun. } \\
2010\end{array}$ \\
\hline Floor on net foreign assets of the SBP* (stock, in millions of U.S. dollars) & 3,953 & 671 & 2,782 & 3,200 & 5,100 & 5,700 & 4,900 \\
\hline Adjusted target & $\ldots$ & 446 & 2,428 & 4,382 & & & \\
\hline Actual & $\ldots$ & 3,132 & 3,982 & 5,706 & & & \\
\hline Ceiling on net domestic assets of the SBP* (stock, in billions of Pakistani rupees) & 1,250 & 1,412 & 1,314 & 1,300 & 1,270 & 1,220 & 1,320 \\
\hline Adjusted target & $\ldots$ & 1,416 & 1,316 & 1,233 & & & \\
\hline Actual & $\ldots$ & 1,201 & 1,183 & 1,141 & & & \\
\hline Ceiling on net government borrowing from SBP* (stock, in billions of Pakistani rupees) & 1,227 & 1,274 & 1,181 & 1,130 & 1,130 & 1,130 & 1,130 \\
\hline Adjusted target & $\ldots$ & 1,274 & 1,181 & 1,130 & & & \\
\hline Actual & $\ldots$ & 1,071 & 1,130 & 1,047 & & & \\
\hline Ceiling on overall budget deficit (cumulative flow, in billions of Pakistani rupees) & 142 & 405 & 562 & 194 & 400 & 604 & 740 \\
\hline Adjusted target & $\ldots$ & 405 & 562 & 173 & & & \\
\hline Actual & $\ldots$ & 405 & 680 & 224 & & & \\
\hline \multicolumn{7}{|l|}{ Ceiling on outstanding stock of short-term public and publicly guaranteed external debt* (in millions } & 1,500 \\
\hline Actual & $\ldots$ & 728 & 652 & 589 & & & \\
\hline $\begin{array}{l}\text { Cumulative ceiling on contracting of nonconcessional medium- and long-term public and publicly } \\
\text { guaranteed external debt* (in millions of U.S. dollars) } 1 /\end{array}$ & 9,500 & 9,500 & 9,500 & 9,500 & 9,500 & 9500 & 9.500 \\
\hline Actual & $\ldots$ & 1,299 & 1,649 & 1,724 & & 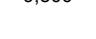 & 0,000 \\
\hline \multicolumn{8}{|l|}{ Accumulation of external payments arrears (continuous performance criterion during the program } \\
\hline period)* (in millions of U.S. dollars) & 0 & 0 & 0 & 0 & 0 & 0 & 0 \\
\hline Actual & $\ldots$ & 0 & 0 & 0 & & & \\
\hline Continuous ceiling on SBP's foreign currency swaps and forward sales* (in millions of U.S. dollars) & 1,900 & 2,750 & 2,750 & 2,500 & 2,500 & 2,500 & 2,500 \\
\hline Actual & $\ldots$ & 1,835 & 1,690 & 2,035 & & & \\
\hline \multicolumn{8}{|l|}{$\begin{array}{l}\text { Memorandum items: } \\
\text { (cumulative, in millions of U.S. dollars, unless otherwise indicated) }\end{array}$} \\
\hline $\begin{array}{l}\text { Net external program financing excluding any budget grants related to Internally Displaced Persons } \\
\text { (IDPs), program portion of Tokyo-related disbursements, and Fund financing to the budget } 3 / \\
\text { Actual }\end{array}$ & -166 & 296 & 737 & $\begin{array}{l}95 \\
-64\end{array}$ & -928 & $-1,082$ & -171 \\
\hline Use of IMF resources by the budget $3 /$ & $\ldots$ & $\ldots$ & $\ldots$ & 715 & 1,072 & 1,430 & 0 \\
\hline Actual & $\cdots$ & $\ldots$ & $\cdots$ & 745 & & & \\
\hline External project grants & 24 & 111 & 176 & 320 & 169 & 530 & 992 \\
\hline Actual & $\cdots$ & $\ldots$ & $\ldots$ & 29 & & & \\
\hline External project grants, excl. IDP grants and Tokyo related grants $3 /$ & $\ldots$ & $\ldots$ & $\ldots$ & 172 & 49 & 70 & 90 \\
\hline Actual & $\cdots$ & $\cdots$ & $\cdots$ & 27 & & & \\
\hline Total IDP grants (project and budget) $3 /$ & $\ldots$ & $\ldots$ & $\ldots$ & 220 & 110 & 310 & 542 \\
\hline Actual & $\ldots$ & $\ldots$ & $\cdots$ & 0 & & & \\
\hline Tokyo-related disbursements, excl. multilateral sources $3 /$ & $\ldots$ & $\ldots$ & $\ldots$ & 342 & 1,177 & 1,962 & 2,220 \\
\hline Actual & $\cdots$ & $\ldots$ & $\ldots$ & 0 & & & \\
\hline Tokyo-related disbursements from multilateral sources $3 /$ & $\cdots$ & $\ldots$ & $\cdots$ & 0 & 0 & 100 & 270 \\
\hline Actual & $\ldots$ & $\ldots$ & $\ldots$ & 0 & & & \\
\hline $\begin{array}{l}\text { Foreign Exchange cash reserve requirement (CRR, incl. special CRR) deposits in SBP (stock in } \\
\text { millions of U.S. dollars) }\end{array}$ & 832 & 745 & 728 & 779 & 800 & 805 & 810 \\
\hline \multicolumn{8}{|l|}{ Weekly cash reserve requirement ratios (in percentage points) } \\
\hline Rupee deposits (less than one year maturity) & 9 & 5 & 5 & 5 & 5 & 5 & 5 \\
\hline Rupee deposits (more than one year maturity) & 0 & 0 & 0 & 0 & 0 & 0 & 0 \\
\hline Foreign currency deposits CRR & 5 & 5 & 5 & 5 & 5 & 5 & 5 \\
\hline Foreign currency deposits special CRR & 15 & 15 & 15 & 15 & 15 & 15 & 15 \\
\hline Increase in SDR allocation (stock in millions of U.S. dollars) & $\ldots$ & $\ldots$ & $\ldots$ & 1,281 & 1,281 & 1,281 & 1,281 \\
\hline
\end{tabular}

Note:

* denotes performance criteria.

1/ Excludes IMF.

2/ In addition to the quantitative performance criteria for end-December, end-March, and end-June the relevant purchases will also be subject to the completion of reviews with the third review to be completed no earlier than November 29, 2009, the fourth review to be completed no earlier than February 27, 2010, and the fifth review to be completed no earlier than May $30,2010$.

$3 /$ To be used in assessing respective adjustors for program targets. 
Table 2. Pakistan: Structural Conditionality, December 2008-July 2010

\begin{tabular}{|c|c|c|}
\hline & Target date & Status \\
\hline \multicolumn{3}{|l|}{ Prior Actions } \\
\hline \multicolumn{3}{|l|}{ Initiate taxpayer audits among the 850 companies identified for audit by either the FBR or external auditors. } \\
\hline Government approval of an electronic payment and tax refund system. & & Met \\
\hline \multicolumn{3}{|l|}{ Structural Benchmarks } \\
\hline A contingency plan for handling problem private banks will be finalized. & end-December 2008 & Met \\
\hline $\begin{array}{l}\text { A full description of required reforms in the area of tax administration, including an } \\
\text { action plan for harmonizing the GST and income tax administration will be finalized. }\end{array}$ & end-December 2008 & Met \\
\hline $\begin{array}{l}\text { In close collaboration with the World Bank, the government will finalize the schedule } \\
\text { for further electricity tariff adjustments during } 2008 / 09 \text {, with a view to eliminating } \\
\text { tariff differential subsidies by end-June } 2009 \text {. }\end{array}$ & end-December 2008 & Met 1/ \\
\hline The SBP's provision of foreign exchange for furnace oil will be eliminated. & February 1, 2009 & Met \\
\hline $\begin{array}{l}\text { In close collaboration with the World Bank, the government will develop a strategy } \\
\text { and a time-bound action plan for the adoption of specific measures to strengthen } \\
\text { the social safety net and improve targeting to the poor. }\end{array}$ & end-March, 2009 & Met \\
\hline The government will prepare a plan for eliminating the inter-corporate circular debt. & end-March, 2009 & Met with a delay \\
\hline The transition to a treasury single account will be completed. & end-June 2010 & Underway $2 /$ \\
\hline $\begin{array}{l}\text { Amendments to the banking legislation will be submitted to Parliament to enhance } \\
\text { the effectiveness of SBP enforcement powers in the area of banking supervision. }\end{array}$ & end-August 2009 & Met with a delay \\
\hline $\begin{array}{l}\text { 1. Government approval of regulations to (i) form new occupational groups within the FBR; } \\
\text { and (ii) revise the structures of Regional Taxpayer Offices and Large Taxpayer Units. } \\
\text { 2. Amendment of all relevant laws and rules. }\end{array}$ & September 15, 2009 & (i) Met; (ii) Met with a delay \\
\hline $\begin{array}{l}\text { The government will submit draft legislative amendments to parliament to harmonize } \\
\text { the income tax and GST laws, including for tax administration purposes. }\end{array}$ & end-September 2009 & Met with a delay $3 /$ \\
\hline Submission of the VAT law to Parliament. & end-December 2009 & \\
\hline $\begin{array}{l}\text { An expedited sales tax refund system will be implemented in all Regional Taxpayer Offices and Large } \\
\text { Taxpayer Units to ensure direct input of refund requests and prompt processing and confirmation of } \\
\text { refunds. }\end{array}$ & end-March 2010 & \\
\hline $\begin{array}{l}\text { An independent review of the Pakistan Improvement of Financial Reporting and Auditing Project (PIFRA), } \\
\text { to improve reporting, lay the basis for commitment controll, and assess in which institution PIFRA should be } \\
\text { located. }\end{array}$ & end-June 2010 & \\
\hline A full implementation of the VAT. & July 1,2010 & \\
\hline
\end{tabular}

1/ The original plan was superseded by a plan that was agreed upon on July 15, 2009 that envisages elimination of tariff differential subsidies by August 2010.

2/ In October 2008, the authorities took steps to prevent accumulation of unspent balances in accounts outside the Federal Consolidated

Fund and made the use of assignment accounts by ministries under the Account No.1 fully operational for budget expenditures.

MoF has collected information about balances held by federal government entities in commercial bank accounts.

The target date has beenrevised from end June-2009.

$3 /$ An ordinance was issued on October 28. It will need to be augmented and legislated at a later stage to be made consistent with the new VAT law that is being prepared. 


\section{AtTachment III. Pakistan: Technical Memorandum of Understanding ON THE Program SuPPORTED UNDER THE STAND-BY ARRANGEMENT}

December 11, 2009

1. With effect from October 1, 2009, this Technical Memorandum of Understanding describes the monitoring arrangements under the SBA-supported program. Throughout, unless otherwise stated, "government" is meant to comprise the federal and provincial governments.

\section{DEFINITIONS OF MONITORING VARIABLES}

2. Valuation of foreign exchange denominated assets, liabilities, and foreign exchange flows. For the purposes of program monitoring under the program, all assets and liabilities as well as debt contracted, denominated in SDRs or in currencies other than the U.S. dollar, will be converted into U.S. dollars at the exchange rates prevailing at test dates, as posted by the State Bank of Pakistan (SBP) on its web site. Net external budget financing and external cash grants will be converted into Pakistani rupees at the exchange rates prevailing at the day of the transaction, as posted by the SBP on its web site, unless otherwise indicated.

3. Reserve money (RM) is defined as the sum of: currency outside scheduled banks (deposit money banks); scheduled banks' domestic cash in vaults; scheduled banks' required and excess rupee; foreign exchange deposits with the SBP; and deposits of the rest of the economy with the SBP, excluding those held by the federal and provincial governments and the SBP staff retirement accounts.

4. Net foreign assets (NFA) of the SBP are defined as the difference between its foreign assets and foreign liabilities. Foreign assets of the SBP consist of gold, foreign exchange, balances held outside Pakistan, foreign securities, foreign bills purchased and discounted, the reserve position with the IMF, and SDR holdings. The definition of foreign assets of the SBP will be consistent with the IMF Data Template on International Reserves and Foreign Currency Liquidity. Gold will be valued at $\$ 20.27$ per troy ounce per fine troy ounce. Foreign liabilities of the SBP include outstanding IMF credits (excluding net IMF financing to the budget as defined in paragraph 10 below), deposits with the SBP of foreign governments, foreign central banks, foreign deposit money banks, international organizations, and foreign nonbank financial institutions (NBFI).

5. Net domestic assets (NDA) of the SBP are defined as the difference between the RM and the NFA of the SBP.

6. Net borrowing from the banking system by the government is defined as the difference between the banking system's claims, on a cash basis, on the federal, provincial, and local governments and the deposits of the federal, provincial, and local governments with 
the banking system, including district government funds balances. For the purposes of this memorandum, claims on government exclude: credit for commodity operations; government deposits exclude outstanding balances in the Zakat Fund; and balances in the various privatization accounts kept by the government in the banking system. The stock of bonds which were issued to banks in substitution of outstanding nonperforming loans to certain public entities, and which are being fully serviced by the government, are included in banking system claims on government. Table 1 summarizes the calculations of net borrowing from the banking system by the government.

7. Net borrowing from the SBP by the government is defined as SBP claims on the government minus government deposits with the SBP. SBP claims on the government include government securities, treasury bills, ways and means advances, treasury currency, and debtor balances. SBP claims on the government exclude accrued profits on government securities. Government deposits with the SBP exclude the Zakat Fund and Privatization accounts (Table 1).

8. The definition of the overall budget deficit (excluding grants) under the program will be the consolidated budget deficit, excluding grants, and including the operations of district governments financed from local funds. It will be measured by the sum of (a) total net financing to the federal, provincial, and local governments; and (b) total external grants to the federal and provincial governments. The former is defined as the sum of (i) net external budget financing (see $\$ 11$ ); (ii) net borrowing from the banking system (as defined above); and (iii) net domestic nonbank financing (see $\$ 12$ ). The total external grants are defined as the sum of project grants, cash external grants for budgetary support, capital grants reflecting the principal amounts of external debt cancellation or swaps, and other grants.

9. Net external program financing is defined to include external privatization receipts; budget support grants; budget support loans from multilateral (other than the IMF, but including World Bank and Asian Development Bank (ADB) budget support and structural adjustment loans), official bilateral budget support loans, and private sector sources (e.g., bonds); rescheduled government debt service and change in stock of external debt service arrears net of government debt amortization due on foreign loans, the latter including any accelerated amortization including related to debt swaps or debt cancellation recorded as capital grants. It also includes foreign loans onlent to financial institutions and companies (public or private) and emergency relief lending. Program financing excludes all external financing counted as reserve liabilities of the SBP (defined above). Amounts projected for net external program financing and external grants are provided in Table 2.

10. Net IMF financing to the budget is defined as the difference between Fund disbursements credited to the budget and repayments from the budget through the Ministry of Finance's dedicated account for Fund financing at the SBP. 
11. Net external budget financing is defined as net external program financing minus privatization receipts, minus budget support grants, plus all other external loans for the financing of public projects or other federal or provincial budget expenditures, plus transfers of external privatization receipts from the privatization account to the budget, plus net IMF financing to the budget. Amounts projected for net external budget financing are provided in Table 2.

12. Net domestic nonbank financing of the budget is defined as follows: domestic privatization receipts transferred from the privatization accounts to the budget, plus the change during each reporting period, in the stock of (i) permanent debt, which consists of nonbank holdings of prize bonds, all federal bonds, and securities; plus (ii) floating debt held by nonbanks; plus (iii) unfunded debt, which consists of National Savings Scheme (NSS) debt, Postal Life Insurance, and the General Provident Fund (GPF); plus (iv) net deposits and reserves received by the government (public accounts deposits); plus (v) any other government borrowing from domestic nonbank sources net of repayments; minus (vi) government deposits with NBFIs. Nonbank holdings of permanent and floating debt is defined as total debt outstanding, as reported by the SBP, minus holdings of banks as per the monetary survey. Total T-bill and other relevant government debt are valued at discount value and exclude accrued interest.

\section{External debt ${ }^{1}$}

13. The performance criterion on contracting or guaranteeing of medium-term and long-term nonconcessional external debt by the government or the SBP applies not only to debt as defined in point No. 9 of the Guidelines on Performance Criteria with Respect to

\footnotetext{
${ }^{1}$ The definition of debt set forth in No. 9 of the guidelines reads as follows: "(a) For the purpose of this guideline, the term "debt" will be understood to mean a current, that is, not contingent, liability, created under a contractual arrangement through the provision of value in the form of assets (including currency) or services, and which requires the obligor to make one or more payments in the form of assets (including currency) or services, at some future point(s) in time; these payments will discharge the principal and/or interest liabilities incurred under the contract. Debts can take a number of forms, the primary ones being as follows: (i) loans, that is, advances of money to obligor by the lender made on the basis of an undertaking that the obligor will repay the funds in the future (including deposits, bonds, debentures, commercial loans, and buyers' credits) and temporary exchanges of assets that are equivalent to fully collateralized loans under which the obligor is required to repay the funds, and usually pay interest, by repurchasing the collateral from the buyer in the future (such as repurchase agreements and official swap arrangements); (ii) suppliers' credits, that is, contracts where the supplier permits the obligor to defer payments until some time after the date on which the goods are delivered or services are provided; and (iii) leases, that is, arrangements under which property is provided which the lessee has the right to use for one or more specified period(s) of time that are usually shorter than the total expected service life of the property, while the lessor retains the title to the property. For the purpose of the guideline, the debt is the present value (at the inception of the lease) of all lease payments expected to be made during the period of the agreement excluding those payments that cover the operation, repair or maintenance of the property. (b) Under the definition of debt set out in point 9 (a) above, arrears, penalties, and judicially awarded damages arising from the failure to make payment under a contractual obligation that constitutes debt are debt. Failure to make payment on an obligation that is not considered debt under this definition (for example, payment on delivery) will not give rise to debt."
} 
Foreign Debt (adopted by the IMF Executive Board on August 24, 2000), but also to commitments contracted or guaranteed for which value has not been received. Excluded from this performance criterion are (i) foreign currency deposit liabilities of the SBP; and (ii) the outstanding stock of debt of Foreign Exchange Bearer Certificates (FEBCs), Deposit Bearer Certificates (DBCs), and Foreign Currency Bearer Certificates (FCBCs). The performance criterion setting a limit on the outstanding stock of short-term external debt refers to debt (as defined in Footnote 1) with original maturity of up to and including one year. Medium- and long-term external debt comprises debt with initial maturity of over one year.

14. Nonconcessional borrowing is defined as borrowing with a grant element of less than 35 percent. ${ }^{2}$ The discount rates used to calculate the grant element will be the six-month and ten-year Commercial Interest Reference Rates (CIRRs) averages, as computed by the Strategy and Policy Review Department of the IMF. Six-month CIRRs are updated midFebruary and mid-August (covering the six-month period preceding the date of update) and the ten-year CIRRs averages are updated mid-December (covering a period of 10 years preceding the date of the update). Six-month CIRRs averages are to be used for loans whose maturity is less than 15 years, while ten-year CIRRs averages are to be used for loans whose maturity is equal or more than 15 years.

15. External payment arrears are defined as unpaid debt service by the government beyond the due date under the contract and any grace period.

16. Tokyo-related disbursements refers to disbursements of donor support (loans and grants) pledged at the donor conference held in Tokyo on April 17, 2009.

\section{AdJustors}

\section{Adjustors related to net external program financing}

17. For paragraphs $18-23$, the net external program financing excludes any budget grants related to Internally Displaced Persons (IDPs), program portion of Tokyo-related disbursements, and Fund financing to the budget. They are projected as follows:

July 2009-December 2009: -\$928 million

July 2009-March 2010: -\$1,082 million

July 2009-June 2010: -\$171 million

18. If the actual cumulative net external program financing in U.S. dollar terms is higher than projected by more than $\$ 500$ million, the excess net external program financing in

\footnotetext{
${ }^{2}$ Following the methodology set out in "Limits on External Debt or Borrowing in Fund ArrangementsProposed Change in Implementation of the Revised Guidelines," April 8, 1996.
} 
U.S. dollar terms is defined as follows: actual external program financing in U.S. dollar terms minus projected net external program financing in U.S. dollar terms minus \$500 million. The excess net external program financing in U.S. dollar terms multiplied by a fixed accounting exchange rate of Rs. 83 per $\$ 1$ represents the excess net external program financing in rupee terms.

19. The ceiling on the consolidated overall budget deficit (excluding grants) will be adjusted upward for the cumulative excess in net external program financing in rupee terms for up to Rs. 73.5 billion at end-December 2009, Rs. 73.5 billion at end-March 2010, and Rs. 73.5 billion at end-June 2010 .

20. The cumulative excess net external program financing in U.S. dollar terms may exceed the projected amounts specified in paragraph 17 for end-December 2009, end-March 2010, and end-June 2010, when converted into U.S. dollar terms at a fixed accounting exchange rate of Rs. 83 per $\$ 1$. In such a case, the cumulative excesses in net external program financing in U.S. dollar terms minus the maximum cumulative amounts specified for end-December 2009, end-March 2010, and end-June 2010 converted into U.S. dollar terms at a fixed accounting exchange rate of Rs. 83 per $\$ 1$ are defined as "surplus net external program financing in U.S. dollar terms." The latter amount multiplied by a fixed accounting exchange rate of Rs. 83 per $\$ 1$ constitutes "surplus net external program financing in rupee terms."

21. The floors on the NFA of the SBP will be adjusted upward by the cumulative surplus net external program financing in U.S. dollar terms as defined above.

22. The ceilings on the NDA of the SBP and net borrowing from the SBP by the government will be adjusted downward by the cumulative surplus net external program financing in rupee terms as defined above.

23. If the actual cumulative net external program financing in U.S. dollar terms is lower than its projected value, the shortfall in net external program financing in U.S. dollar terms is defined as the difference between its projected and actual values in U.S. dollar terms. In such a case:

a. The floor on the NFA of the SBP is adjusted downward by the cumulative shortfall in net external program financing in U.S. dollar terms.

b. The ceiling on the NDA of the SBP is adjusted upward by the cumulative shortfall in net external program financing in U.S. dollar terms converted into rupees at a fixed accounting exchange rate of Rs. 83 per $\$ 1$.

c. The ceiling on net borrowing from the SBP by the government is not subject to adjustment. 


\section{Adjustors related to Tokyo-related disbursements (excluding multilateral sources)}

24. Tokyo-related disbursements (excluding multilateral sources) are projected as follows:

July 2009-December 2009: \$1,177 million

July 2009-March 2010: \$1,962 million

July 2009-June 2010: \$2,220 million

25. If the actual cumulative gross Tokyo-related disbursements (excluding multilateral sources) are lower than the projected amount, the difference between their projected and actual values in U.S. dollar terms is defined as the shortfall in Tokyo-related disbursements (excluding multilateral sources) in U.S. dollar terms. The shortfall in Tokyo-related disbursements (excluding multilateral sources) in U.S. dollar terms multiplied by a fixed accounting exchange rate of Rs. 83 per $\$ 1$ represents the shortfall in Tokyorelated disbursements (excluding multilateral sources) in rupee terms. In such a case:

a. The ceiling on the consolidated overall budget deficit (excluding grants) is adjusted downward up to Rs. 29.49 billion at end-September 2009, Rs. 58.97 billion at end-December 2009, Rs. 58.97 billion at end-March 2010, and Rs. 58.97 billion at end-June 2010 (equivalent to $\$ 0.71$ billion valued at Rs. 83 per $\$ 1$ ) for shortfall in Tokyo-related disbursements (excluding multilateral sources) in rupee terms in excess of Rs. 117.94 billion.

b. The floor on the NFA of the SBP is adjusted downward by the amount equivalent to the cumulative shortfall in Tokyo-related disbursements (excluding multilateral sources) in U.S. dollar terms.

c. The ceiling on the NDA of the SBP is adjusted upward by the amount equivalent to the cumulative shortfall in Tokyo-related disbursements (excluding multilateral sources) in rupee terms.

\section{Adjustors related to Tokyo-related disbursements from multilateral sources}

26. Tokyo-related disbursements from multilateral sources are projected as follows:

July 2009-December 2009: \$0 million

July 2009-March 2010: \$100 million

July 2009-June 2010: \$270 million

27. If the actual cumulative gross Tokyo-related disbursements from multilateral sources are lower than the projected amount, the difference between their projected and actual values 
in U.S. dollar terms is defined as the shortfall in Tokyo-related disbursements from multilateral sources in U.S. dollar terms. The shortfall in related disbursements Tokyorelated disbursements from multilateral sources in U.S. dollar terms multiplied by a fixed accounting exchange rate of Rs. 83 per $\$ 1$ represents the shortfall in Tokyo-related disbursements from multilateral sources in rupee terms. In such a case:

a. The ceiling on the consolidated overall budget deficit (excluding grants) is adjusted downward by the shortfall in Tokyo-related disbursements from multilateral sources in rupee terms.

b. The floor on the NFA of the SBP is adjusted downward by the amount equivalent to the cumulative shortfall in Tokyo-related disbursements from multilateral sources in U.S. dollar terms.

c. The ceiling on the NDA of the SBP is adjusted upward by the amount equivalent to the cumulative shortfall in Tokyo-related disbursements from multilateral sources in rupee terms.

d. The ceiling on net borrowing from the SBP by the government will not be adjusted.

\section{Adjustor related to net IMF financing to the budget}

28. Net IMF financing to the budget is projected as follows:

July 2009-December 2009: \$1,072 million

July 2009-March 2010: \$1,430 million

July 2009-June 2010: \$0 million

29. If actual net Fund financing to the budget in U.S. dollar terms is different from the projected amount, the difference in net Fund financing to the budget in U.S. dollar terms is defined as the difference in actual net Fund financing converted into U.S. dollar by the actual SDR/U.S. dollar exchange rates and the projected amount. In such a case,

a. The floor on the NFA of the SBP will be adjusted by the difference in Fund financing to the budget in U.S. dollar terms (upward if the difference is positive, downward if the difference is negative).

b. The ceiling on the NDA of the SBP will be adjusted by subtracting the difference in Fund financing to the budget in U.S. dollar terms converted into rupees at a fixed accounting exchange rate of Rs. 83 per $\$ 1$ (downward if the difference is positive, upward if the difference is negative). 


\section{Adjustor related to SDR allocations}

30. If actual SDR allocation in U.S. dollar terms is different than projected amount (Table 2), the difference in SDR allocations in U.S. dollar terms is defined as the difference in actual disbursements converted into U.S. dollar by the actual SDR/dollar exchange rates and the projected disbursement in U.S. dollar terms. In such a case,

a. The floor on the NFA of the SBP will be adjusted by the difference in SDR allocations in U.S. dollar terms (upward if the difference is positive, downward if the difference is negative).

b. The ceiling on the NDA of the SBP will be adjusted by subtracting the difference in SDR allocations in U.S. dollar terms converted into rupees at a fixed accounting exchange rate of Rs. 83 per $\$ 1$ (downward if the difference is positive, upward if the difference is negative).

\section{Adjustor related to external project grants excluding IDP grants and Tokyo-related grants}

31. If the amount of external project grants excluding IDP grants and Tokyo-related grants, is higher or lower than assumed under the program (see Table 2), the ceiling on the consolidated overall budget deficit (excluding grants) will be adjusted upward or downward for the cumulative excess or shortfall in these grants. Where applicable, this adjustor will not be applied in addition to any adjustment to the consolidated overall budget deficit (excluding grants) that is made under paragraphs 19, 25a, and 27a.

\section{Adjustor related to total IDP grants (project and budget)}

32. If the total amount of IDP grants (including project and budget grants), is higher or lower than assumed under the program (see Table 2), the ceiling on the consolidated overall budget deficit (excluding grants) will be adjusted upward or downward for the cumulative excess or shortfall in these grants. Any shortfall in the period July - December 2009 will not be applied to the ceiling for this period. Where applicable, this adjustor will not be applied in addition to any adjustment to the consolidated overall budget deficit (excluding grants) that is made under paragraphs 19, 25a, and 27a.

\section{Adjustor related to changes in regulations on required reserves}

33. The ceilings on the NDA of the SBP will also be adjusted downward/upward by the amount of (i) banks' Pakistani rupee reserves freed/seized by any reduction/increase of the daily CRR relative to the baseline assumption; and (ii) any reduction/increase in the reservable deposit base that is related to definitional changes, as per the following formula: $\Delta \mathrm{NDA}=\Delta \mathrm{rB}_{0}+\mathrm{r}_{0} \Delta \mathrm{B}+\Delta \mathrm{r} \Delta \mathrm{B}$, where $\mathrm{r}_{0}$ denotes the reserve requirement ratio prior to any change; $\mathrm{B}_{0}$ denotes the level of the reservable deposits in the initial definition; $\Delta \mathrm{r}$ is the 
change in the reserve requirement ratio; and $\Delta \mathrm{B}$ denotes the change in the reservable deposits as a result of definitional changes. In case of significant liquidity and other financial sector pressures, the SBP will engage in consultations with the IMF staff in order to reach understanding on appropriate monetary policy response.

\section{Adjustor related to the SBP's net position under foreign exchange forwards and swaps}

34. An adjustor to the NFA target of the SBP will be implemented to reflect changes in the SBP's net position under foreign exchange forwards and swaps. Specifically, the NFA target of the SBP will be adjusted upward/downward by the amount of the increase/decrease in the net SBP's position under foreign exchange forwards and swaps. The SBP's net exposure under foreign exchange forwards and swaps was $\$ 2.035$ billion at end-September 2009. The maximum SBP's net exposure under foreign exchange forwards and swaps is capped at \$2.5 billion for end-December 2009, end-March 2010, and end-June 2010.

\section{Adjustor related to foreign currency deposits of resident banks with the SBP}

35. An adjustor to the NFA target of the SBP will be implemented to reflect changes in foreign currency deposits of resident banks. Specifically, the NFA target of the SBP will be adjusted upward/downward by the amount of increase/decrease in foreign currency deposits of resident banks with the SBP. The stock of foreign currency deposits of resident banks with the SBP was $\$ 779$ million at end-September 2009.

\section{Program Reporting ReQuirements}

36. The following information, including any revisions to historical data, will be provided to the Middle East and Central Asia Department of the IMF through the office of the Resident Representative of the IMF in Pakistan, within the timeframe indicated:

- Monthly provisional statements on federal tax and nontax revenue, within one month.

- Deposits into and withdrawals from the privatization accounts for each quarter, within one month. Withdrawals will be reported with the following breakdown (i) those which constitute budgetary use of privatization proceeds; (ii) those which constitute costs of privatization; and (iii) other (with explanation of the purpose of other withdrawals), as well as with the breakdown between domestic and external privatization receipts.

- Quarterly statements on budgetary capital receipts and disbursements, including repayments of bonds, recovery of loans from provinces and "others," within two months.

- $\quad$ Monthly (unreconciled) provisional data on federal expenditure and net lending (with separate data on disbursements and repayments), within one month. 
- Quarterly statement on consolidated budgetary expenditure, with federal data approved by the Accountant General Pakistan Revenue (AGPR), within two months.

- Quarterly numbers on expenditure on social programs.

- Quarterly data on the stock of domestic government debt, broken down by instrument, within one month (Table 3).

- Quarterly data on WAPDA receivables within one month.

- Monthly data on Outstanding Audited Price Differential Claims.

- $\quad$ Monthly data on external budget financing, including (i) loan-by-loan program disbursements in U.S. dollar terms and rupee terms converted at exchange rates prevailing at the time of each transaction; (ii) cumulative amortization in U.S. dollar terms and rupee terms converted at exchange rates prevailing at the time of each transaction; and (iii) cumulative project loan disbursements in U.S. dollar terms and in rupees converted at exchange rates prevailing at the time of each transaction. Tokyo and IDP related disbursement should be indicated separately.

- $\quad$ Monthly data on Banks' Budgetary Support (Table 1) within one month.

- $\quad$ The following monthly monetary data on a last-Saturday basis within two weeks:

(i) monetary survey;

(ii) accounts of the SBP;

(iii) consolidated accounts of the scheduled banks;

(iv) banks' lending to the government;

(v) detailed table on net foreign assets (both for the SBP and scheduled banks); and

(vi) detailed table of scheduled banks' reserves with the SBP.

- $\quad$ The same tables as in the preceding item, but on an end-month and end-quarter basis (last business day), both at current and program exchange rates, within one month.

- $\quad$ The SBP Table on outstanding stock of foreign currency deposits, amended to include the classification of new FCA according to the residency of the holder.

- Daily data on exchange rates (interbank, retail market, and Telegraphic Transfers for SBP purchases in the retail market), SBP's sales and purchases in the foreign exchange markets, swaps and forward outright sales, within two business days.

- Monthly data on the outstanding stock of the SBP's forward foreign currency operations, including swaps and outright forward sales and purchases, within two weeks. The terms of any new transactions (including rollover/renewal of existing ones) will also be provided. 
- Monthly data on the SBP's foreign exchange reserves, with details on the currencies, instruments, and institutions in which the reserves are held, within one month.

- Monthly data on SBP direct or bridge loans to nationalized banks in the context of the restructuring and privatization operation, within four weeks.

- Monthly data on any other quasi-fiscal operations undertaken by the SBP, on behalf of the government.

- Monthly data on SBP holding of discounted export finance credit under the export finance scheme, within one month.

- Monthly data on outstanding credit to agriculture under the Agriculture Mandatory Credit Targets, within one month.

- $\quad$ The following data on external debt, within one month:

(i) Quarterly stock of public- and publicly-guaranteed external debt (including deferred payments arrangements), by maturity (initial maturities of up to and including one year, and over one year), by creditor and by debtor (central government and publicly guaranteed);

(ii) Quarterly contracting or guaranteeing of nonconcessional medium- and long-term government debt; and

(iii) Information on any rescheduling on public- and publicly-guaranteed debt reached with creditors.

- Quarterly data on external payments arrears on public and publicly guaranteed debt with details as in (i) of the preceding item within one month.

- $\quad$ Copies of new or revised ordinances/circulars regarding changes in tax policy, tax administration, foreign exchange market regulations, and banking regulations no later than three days after official issuance, or notification that ordinances have been posted on the Federal Board or Revenue (FBR) and SBP websites.

- Copies of official notification of changes in gas and electricity tariffs and any surcharges (automatic or structural) and in ex-refinery petroleum product prices as well as of gas and petroleum surcharges/levies.

- Monthly data on the import parity prices as well as central depot prices of the six major oil products, within one month.

- Quarterly data on KESC and WAPDA loans and debt outstanding, within one month. 
- Upon the adoption of the plan for the elimination of inter-corporate circular debt, monthly reports on inter-corporate circular debt will be reported within one month.

- Information on new liabilities incurred or guaranteed by the government in settling the circular-debt, including government guarantees of Term Financing Certificates (TFCs) issued by Pakistan Electric Power Company (PEPCO). The information will include the size of the government exposure, the duration of the guarantee or claim, and any other provisions relevant for the government's exposure.

- Information on the transactions between the Ministry of Finance and the SBP with regard to the use of Fund resources for budget purposes on monthly basis. 
Table 1. Pakistan Budgetary Support

(In million Rupees)

\begin{tabular}{|c|c|c|c|c|}
\hline & & 30-Jun-09 & 30-Sep-09 & 31-Oct-09 \\
\hline \multirow[t]{20}{*}{ A. } & Central Government & $1,849,101$ & $1,867,809$ & $1,924,524$ \\
\hline & Scheduled Banks & 737,490 & 861,515 & 854,686 \\
\hline & Government Securities & 214,164 & 225,290 & 223,538 \\
\hline & Treasury Bills & 756,955 & 890,690 & 889,222 \\
\hline & Government Deposits & $-233,630$ & $-254,465$ & $-258,074$ \\
\hline & State Bank & $1,111,612$ & $1,006,294$ & $1,069,838$ \\
\hline & Government Securities & 3,144 & 3,144 & 3,144 \\
\hline & Accrued Profit on MRTBs & 35,131 & 33,126 & 31,306 \\
\hline & Treasury Bills & $1,107,858$ & $1,010,757$ & $1,025,957$ \\
\hline & MTFBs purchased for replenishment of cash balances & $1,098,349$ & $1,001,248$ & $1,016,448$ \\
\hline & Adhoc Treasury Bills & 0 & 0 & 0 \\
\hline & Ways and Means Advances & 0 & 0 & 0 \\
\hline & Treasury Currency & 8,153 & 8,153 & 8,153 \\
\hline & Debtor Balances (Excl. Zakat Fund) & 0 & 0 & 0 \\
\hline & Government Deposits & $-48,137$ & $-54,348$ & $-4,185$ \\
\hline & (Excl. Zakat and Privatization Fund) & 0 & 0 & 0 \\
\hline & Special Account-Debt Repayment & 0 & 0 & 0 \\
\hline & Payment to HBL on a/c of HC\&EB & -287 & -287 & -287 \\
\hline & Adjustment for use of Privatization Proceeds & & & \\
\hline & for Debt Retirement & 5,749 & 5,749 & 5,749 \\
\hline \multirow[t]{12}{*}{ B. } & Provincial Governments & $-168,079$ & $-148,711$ & $-174,317$ \\
\hline & Scheduled Banks $(a+b-c)$ & $-221,114$ & $-222,448$ & $-225,519$ \\
\hline & Government Securities & 0 & 0 & 0 \\
\hline & Advances to Punjab Government for Cooperatives & 1,024 & 1,024 & 1,024 \\
\hline & Government Deposits & $-222,138$ & $-223,472$ & $-226,544$ \\
\hline & State Bank & 53,035 & 73,737 & 51,203 \\
\hline & Debtor Balances (Excl. Zakat Fund) & 75,381 & 101,091 & 92,010 \\
\hline & Ways and Means Advances & 0 & 0 & 0 \\
\hline & Government Deposits (Excl.Zakat Fund) & $-22,346$ & $-27,354$ & $-40,807$ \\
\hline & Net Govt. Budgetary Borrowings from the Banking system & $1,681,022$ & $1,719,098$ & 1750207.59 \\
\hline & Through SBP & $1,164,647$ & $1,080,031$ & $1,121,041$ \\
\hline & Through Scheduled Banks & 516,375 & 639,068 & 629,167 \\
\hline \multicolumn{5}{|c|}{ Memorandum Items } \\
\hline & Accrued Profit on SBP holding of MRTBs & 35,131 & 33,126 & 31,306 \\
\hline & Scheduled banks ' deposits of Privitization Commission & $-1,580$ & $-2,380$ & $-1,963$ \\
\hline & Outstanding amount of MTBs (Primary market; discounted value) & 739,475 & 871,341 & 872,808 \\
\hline \multicolumn{5}{|c|}{ Net Govt. Borrowings (Cash basis) } \\
\hline & From Banking System & $1,629,991$ & $1,669,004$ & $1,704,451$ \\
\hline & From SBP & $1,129,516$ & $1,046,905$ & $1,089,735$ \\
\hline & From Scheduled Banks & 500,475 & 622,098 & 614,716 \\
\hline
\end{tabular}

Source: State Bank of Pakistan 
Table 2. Pakistan: External Financing For Budget for 2008/09 and 2009/10 (In millions of U.S. dollars)

\begin{tabular}{|c|c|c|c|c|c|c|c|c|c|c|}
\hline & $\begin{array}{r}\text { Act. } \\
\text { Jul.-Sep. }\end{array}$ & $\begin{array}{r}\text { Act. } \\
\text { Oct.-Dec. } \\
\end{array}$ & $\begin{array}{r}\begin{array}{r}\text { Act. } \\
\text { Jan.-Mar. }\end{array} \\
2008 / 09\end{array}$ & $\begin{array}{r}\text { Act. } \\
\text { Apr.-Jun. } \\
\end{array}$ & $\begin{array}{r}\text { Act. } \\
\text { Total } \\
\end{array}$ & $\begin{array}{r}\text { Act. } \\
\text { Jul.-Sep. } \\
\end{array}$ & $\begin{array}{r}\text { Proj. } \\
\text { Oct.-Dec. } \\
\end{array}$ & $\begin{array}{r}\begin{array}{r}\text { Proj. } \\
\text { Jan.-Mar. }\end{array} \\
2009 / 10\end{array}$ & $\begin{array}{r}\text { Proj. } \\
\text { Apr.-Jun. } \\
\end{array}$ & $\begin{array}{l}\text { Proj. } \\
\text { Total } \\
\end{array}$ \\
\hline Project Aid & 248 & 331 & 268 & 319 & 1,165 & 231 & 266 & 1,236 & 999 & 2,732 \\
\hline Grants, of which & 23 & 21 & 50 & 2 & 96 & 27 & 22 & 285 & 265 & 599 \\
\hline Regular & & & & & & 27 & 22 & 21 & 20 & 90 \\
\hline Tokyo pledge & 0 & 0 & 0 & 0 & 0 & 0 & 0 & 130 & 195 & 325 \\
\hline IDP grants & 0 & 0 & 0 & 0 & 0 & 0 & 0 & 134 & 50 & 184 \\
\hline Earth Quake grants & 1 & 2 & 3 & 74 & 80 & 2 & 8 & 10 & 15 & 35 \\
\hline Regular & & & & & & 86 & 45 & 45 & 210 & 386 \\
\hline Tokyo pledge & 0 & 0 & 0 & 0 & 0 & 0 & 0 & 0 & 0 & 0 \\
\hline Earthquake & 7 & 21 & 0 & 20 & 49 & 0 & 0 & 0 & 0 & 0 \\
\hline$A D B$, of which & 111 & 85 & 53 & 75 & 324 & 74 & 73 & 76 & 77 & 300 \\
\hline Regular & & & & & & 74 & 73 & 76 & 77 & 300 \\
\hline Tokyo pledge & 0 & 0 & 0 & 0 & 0 & 0 & 0 & 0 & 0 & 0 \\
\hline Earthquake & 7 & 9 & 5 & 6 & 27 & 0 & 0 & 0 & 0 & 0 \\
\hline Other bilateral project loan, of which & 52 & 44 & 68 & 43 & 207 & 42 & 118 & 720 & 432 & 1,312 \\
\hline Regular & & & & & & 42 & 118 & 120 & 156 & 436 \\
\hline Tokyo pledge & 0 & 0 & 0 & 0 & 0 & 0 & 0 & 600 & 276 & 876 \\
\hline Other Earthquake Loans & 0 & 0 & 0 & 54 & 54 & 0 & 0 & 0 & 0 & 0 \\
\hline Program Loans & 494 & 811 & 677 & 815 & 2,797 & 414 & 36 & 600 & 1,100 & 2,150 \\
\hline World Bank & 0 & 0 & 485 & 224 & 709 & 264 & 36 & 0 & 300 & 600 \\
\hline $\mathrm{ADB}$ & 494 & 100 & 96 & 492 & 1,182 & 150 & 0 & 400 & 600 & 1,150 \\
\hline Tokyo pledge, of which & 0 & 0 & 0 & 51 & 51 & 0 & 0 & 0 & 0 & 0 \\
\hline World Bank & 0 & 0 & 0 & 0 & 0 & 0 & 0 & 0 & 0 & 0 \\
\hline ADB & 0 & 0 & 0 & 51 & 51 & 0 & 0 & 0 & 0 & 0 \\
\hline IDB & 0 & 561 & 77 & 18 & 656 & 0 & 0 & 200 & 200 & 400 \\
\hline Short-term commercial & 0 & 150 & 19 & 31 & 200 & 0 & 0 & 0 & 0 & 0 \\
\hline Budgetary Grants & 40 & 30 & 56 & 5 & 131 & 0 & 38 & 62 & 0 & 100 \\
\hline Privatization & 0 & 0 & 0 & 0 & 0 & 0 & 0 & 133 & 133 & 266 \\
\hline GDRs & 0 & 0 & 0 & 0 & 0 & 0 & 0 & 0 & 0 & 0 \\
\hline Securitization/China & 0 & 0 & 500 & 0 & 500 & 0 & 0 & 0 & 0 & 0 \\
\hline IDP budget grants & 0 & 0 & 0 & 10 & 10 & 0 & 0 & 0 & 0 & 0 \\
\hline Saudi Arabia (Tokyo pledge) & $\ldots$ & $\ldots$ & $\ldots$ & $\ldots$ & $\ldots$ & 0 & 0 & 200 & 0 & 200 \\
\hline Other grants & $\ldots$ & $\ldots$ & $\ldots$ & $\ldots$ & $\ldots$ & 0 & 0 & 0 & 0 & 0 \\
\hline Budget financing from the Fund & $\ldots$ & $\ldots$ & $\ldots$ & $\ldots$ & $\ldots$ & 745 & 354 & 354 & -733 & 719 \\
\hline Amortization & 700 & 802 & 810 & 379 & 2,691 & 478 & 938 & 949 & 322 & 2,687 \\
\hline Medium and Long-term & 538 & 258 & 760 & 277 & 1,833 & 395 & 279 & 944 & 322 & 1,940 \\
\hline Euro bonds & 22 & 0 & 500 & 0 & 522 & 22 & 0 & 600 & 0 & 622 \\
\hline$I D B>1$ year & 200 & 0 & 0 & 0 & 200 & 0 & 0 & 0 & 0 & 0 \\
\hline Commercial & 0 & 116 & 50 & 0 & 166 & 0 & 116 & 0 & 0 & 116 \\
\hline \multicolumn{11}{|l|}{$\begin{array}{l}\text { Memorandum items: } \\
\text { (cumulative from the beginning of fiscal year) }\end{array}$} \\
\hline Net budget financing & 82 & 452 & 1,142 & 1,903 & & 912 & 668 & 2,303 & 3,480 & 3,480 \\
\hline $\begin{array}{l}\text { Net program financing for the budget (excl. Tokyo related disbursements, IDP } \\
\text { grants, Fund financing to the budget) } 1 /\end{array}$ & -166 & -127 & 296 & 737 & & -64 & -928 & $-1,082$ & -171 & -171 \\
\hline Tokyo-related disbursements (excl. multilateral sources) 1/ & 0 & 0 & 0 & 0 & & 342 & 1,177 & 1,962 & 2,220 & 2,220 \\
\hline Tokyo-related disbursements from multilateral sources $1 /$ & 0 & 0 & 0 & 0 & & 0 & 0 & 100 & 270 & 270 \\
\hline Total project grants & & & & & & 29 & 169 & 530 & 992 & 992 \\
\hline External project grants, excl. IDP grants and Tokyo related grants 1/ & 24 & 47 & 100 & 176 & & 27 & 49 & 70 & 90 & 90 \\
\hline Total grants for IDP (budget and project) $1 /$ & 0 & 0 & 0 & 0 & & 0 & 110 & 310 & 542 & 542 \\
\hline Increase in SDR allocation & & & & & & 1,281 & 1,281 & 1,281 & 1,281 & 1,281 \\
\hline
\end{tabular}

Sources: Pakistani authorities; and Fund staff projections.

$1 /$ To be used in assessing respective adjustors for program targets, rather than the projected amount above in the table 
Table 3. Pakistan: Total Outstanding Domestic Debt

(In billion Rupees)

\begin{tabular}{|c|c|c|c|}
\hline & Jun-08 & Jun-09 & Sep-09 \\
\hline A. Permanent Debt & 608.0 & 678.0 & 717.1 \\
\hline Market Loans & 3.0 & 2.9 & 2.9 \\
\hline Federal Government Bonds & 9.0 & 7.2 & 7.2 \\
\hline Income Tax Bonds & 0.0 & 0.0 & 0.0 \\
\hline Government Bonds (L.R.-1977) & 0.0 & 0.1 & 0.1 \\
\hline Special Government Bonds For SLIC (Capitalization) & 1.0 & 0.6 & 0.6 \\
\hline GOP ljara Sukuk 3 years & 0.0 & 27.8 & 42.2 \\
\hline $\begin{array}{l}\text { Government Bonds (issued to HBL for settlement of CBR } \\
\text { Refund) }\end{array}$ & 0.0 & 0.0 & 0.0 \\
\hline Bearer National Fund Bonds(BNFB) & 0.0 & 0.0 & 0.0 \\
\hline BNFB Roll Over-II & 0.0 & 0.0 & 0.0 \\
\hline Special National Fund Bonds & 0.0 & 0.0 & 0.0 \\
\hline Federal Investment Bonds (Auction) & 1.0 & 1.0 & 0.0 \\
\hline Federal Investment Bonds (TAP) & 0.0 & 0.0 & 0.0 \\
\hline Pakistan Investment Bonds (PIBs) & 412.0 & 441.0 & 456.7 \\
\hline Prize Bonds & 183.0 & 197.4 & 207.4 \\
\hline B. Floating Debt & $1,637.0$ & $1,904.0$ & $1,970.4$ \\
\hline Treasury Bills (3 Months) & 0.0 & 0.0 & 0.0 \\
\hline Market Treasury Bills (auction) & 537.0 & 796.1 & 959.6 \\
\hline MTBs for Replenishment* & $1,100.0$ & $1,107.9$ & $1,010.8$ \\
\hline C. Unfunded Debt & $1,020.0$ & $1,270.5$ & $1,322.5$ \\
\hline Defense Savings Certificates & 285.0 & 257.2 & 233.2 \\
\hline National Deposit Certificates & 0.0 & 0.0 & 0.0 \\
\hline Khas Deposit Certificates & 0.0 & 0.3 & 0.3 \\
\hline Special Savings Certificates (Reg) & 160.0 & 288.8 & 318.7 \\
\hline Special Savings Certificates (Bearer) & 0.0 & 0.3 & 0.3 \\
\hline Regular Income Certificates & 51.0 & 91.1 & 102.7 \\
\hline Premium Saving Certificates & 0.0 & 0.0 & 0.0 \\
\hline Bahbood Savings Certificates & 229.0 & 307.5 & 320.7 \\
\hline Khas Deposit Accounts & 0.0 & 0.3 & 0.3 \\
\hline National Deposit Accounts & 0.0 & 0.0 & 0.0 \\
\hline Savings Accounts & 28.0 & 16.8 & 15.6 \\
\hline Special Savings Accounts & 67.0 & 88.6 & 108.4 \\
\hline Mahana Amdani Accounts & 2.0 & 2.4 & 2.3 \\
\hline Pensioners' Benefit Accounts & 88.0 & 109.9 & 113.9 \\
\hline Postal Life Insurance & 67.0 & 67.1 & 67.1 \\
\hline GP Fund & 43.0 & 40.1 & 39.1 \\
\hline D. Total $(A+B+C)$ & $3,266.0$ & $3,852.6$ & $4,010.0$ \\
\hline
\end{tabular}

Note: * Inclusive of outright sale of MTBs to commercial banks.

Source: State Bank of Pakistan (Statistics \& DWH Department). 


\section{INTERNATIONAL MONETARY FUND}

\section{PAKISTAN}

\section{Third Review under the Stand-By Arrangement \\ Informational Annex}

Prepared by Middle East and Central Asia Department

December 14, 2009

Contents

Page

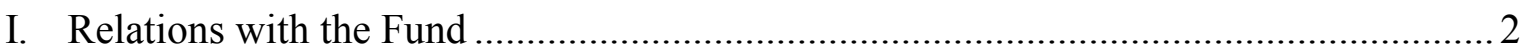

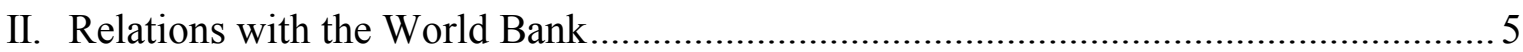




\section{AnNeX I. PAKistan: Relations With THE FUnd}

As of October 31, 2009

I. Membership Status: Joined: 07/11/1950; Article VIII

II. General Resources Account:

$\underline{\text { SDR Million }}$

Quota

$1,033.70$

\% Quota

Fund Holdings of Currency

$4,436.22$

100.00

Reserve position in Fund

0.12

429.16

0.01

III. SDR Department:

Net cumulative allocation

$\underline{\text { SDR Million }}$

\% Allocation

988.56

100.00

Holdings

903.13

91.36

IV. Outstanding Purchases and Loans:

$\underline{\text { SDR Million }}$

\% Quota

Stand-by Arrangements

$3,402.64$

329.17

PRGF arrangements

654.67

63.33

V. Latest Financial Arrangements:

\begin{tabular}{|c|c|c|c|c|}
\hline Type & $\begin{array}{l}\text { Approval } \\
\text { Date }\end{array}$ & $\begin{array}{l}\text { Expiration } \\
\text { Date }\end{array}$ & $\begin{array}{c}\text { Amount Approved } \\
\text { (SDR Million) }\end{array}$ & $\begin{array}{c}\text { Amount Drawn } \\
\text { (SDR Million) }\end{array}$ \\
\hline Stand-By & $11 / 24 / 2008$ & $10 / 23 / 2010$ & $7,235.90$ & $3,402.64$ \\
\hline PRGF & $12 / 06 / 2001$ & $12 / 05 / 2004$ & $1,033.70$ & 861.42 \\
\hline Stand-By & $11 / 29 / 2000$ & 09/30/2001 & 465.00 & 465.00 \\
\hline
\end{tabular}

VI. Projected Payments to the Fund ${ }^{1,2}$

(SDR Million; based on existing use of resources and present holdings of SDRs):

\begin{tabular}{lrrrrr} 
& \multicolumn{5}{c}{ Forthcoming } \\
\cline { 2 - 6 } & $\mathbf{2 0 0 9}$ & $\mathbf{2 0 1 0}$ & $\mathbf{2 0 1 1}$ & $\mathbf{2 0 1 2}$ & $\mathbf{2 0 1 3}$ \\
Principal & 43.07 & 172.28 & 172.28 & $1,418.11$ & $1,787.46$ \\
Charges/Interest & 13.79 & 52.53 & 51.66 & 42.21 & 20.43 \\
Total & 56.86 & 224.81 & 223.95 & $1,460.32$ & $1,807.89$
\end{tabular}

\footnotetext{
${ }^{1}$ This schedule presents all currently scheduled payments to the IMF, including repayment expectations and repayment obligations. The IMF Executive Board can extend repayment expectations (within predetermined limits) upon request by the member if its external payment position is not strong enough to meet the expectations without undue hardship or risk.

${ }^{2}$ When a member has overdue financial obligations outstanding for more than three months, the amount of such arrears will be shown in this section.
} 


\section{Exchange System}

On May 19, 1999, the dual exchange rate system was unified, with all international transactions conducted at the interbank market exchange rate (FIBR). The Fund classifies Pakistan's exchange rate regime as floating. Pakistan has accepted the obligations of Article VIII. It maintains a restriction subject to Fund approval in the form of a 25 percent limit on advance payments for imports of goods. The authorities eliminated cash margin requirements on letters of credit in June 2009.

\section{Last Article IV Consultation}

The last Article IV consultation (Country Report 09/123) was discussed by the Executive Board on March 23, 2009.

\section{Safeguards Assessments}

In accordance with the Fund's safeguard assessment policy, the State Bank of Pakistan (SBP) was subject to a full safeguards assessment under the Stand-By Arrangement that was approved in November 2008. This update assessment included a safeguard mission to the SBP in late January 2009. The assessment found improved transparency of the audited financial statements, coupled with stronger governance oversight by the Central Board and the Audit Committee. The SBP embarked on a modernization program that saw the implementation of a new organization structure, IT systems, and the recruitment of a qualified professional staff. Amendments to the SBP law to enhance the SBP's operational independence have been submitted to Parliament. Also, staff noted the need to strengthen controls over program data reporting to the Fund through an independent review mechanism. An update safeguards assessment is underway in relation to the augmentation of access under the Stand-By Arrangement.

\section{FSAP Participation and ROSCs}

\begin{tabular}{|l|c|l|}
\hline Fiscal Transparency Module & $11 / 28 / 2000$ & (www.imf.org) \\
\hline Fiscal Transparency Module_Update & $11 / 22 / 2004$ & Country Report No. 04/416 \\
\hline $\begin{array}{l}\text { Financial System Stability Assessment } \\
\text { Financial Sector Assessment Program }\end{array}$ & $6 / 23 / 2004$ & Country Report No. 04/215 \\
\hline $\begin{array}{l}\text { Data Module and Detailed Assessment } \\
\text { Using Quality Assessment Framework }\end{array}$ & $11 / 29 / 2004$ & Country Report No. 04/398 \\
\hline $\begin{array}{l}\text { Data Module, Reassessment of Monetary } \\
\text { Statistics and Detailed Assessment Using } \\
\text { Quality Assessment Framework }\end{array}$ & $2 / 2 / 2007$ & Country Report No. 07/74 \\
\hline
\end{tabular}




\begin{tabular}{|l|c|l|}
\hline $\begin{array}{l}\text { Fiscal Transparency Module-Draft } \\
\text { Update }\end{array}$ & $2 / 13 / 2007$ & Country Report No. 08/129 \\
\hline $\begin{array}{l}\text { Financial System Stability Assessment, } \\
\text { Financial Sector Assessment Program } \\
\text { Update }\end{array}$ & $9 / 22 / 2008$ & In progress \\
\hline
\end{tabular}

\section{Recent Technical Assistance}

\section{FAD}

January 2002: Fiscal data management, quality, and transparency.

January 2003: Tax administration.

February/March 2003: Customs administration.

April 2004: Fiscal reporting.

April 2007: Public financial management.

July and November 2009: Valued Added Tax law.

\section{MCM}

November/December 2004: Public debt reform and capacity building program (joint with World Bank).

March/April 2005: Development of the Insurance Sector.

December 2006: Monetary policy framework.

April 2007: Monetary policy framework, the SBP's balance sheet, and the Banking Services Corporation.

\section{STA}

February 2002: External sector statistics/SDDS subscription.

April/May 2005: National accounts and consumer price statistics.

May 2007: Statistics on the international investment position.

October 2009: Multisector statistics (remote technical assistance).

\section{LEG}

July 2008: Deposit Protection Fund.

July 2008: Central Bank Law.

August 2008: Banking Law.

\section{Resident Representative}

A resident representative has been stationed in Islamabad since August 1991. The current resident representative Mr. Paul Ross took up his post in October 2008. 


\section{AnNeX II. Pakistan: Relations With the World Bank Group}

Thomas Buckley, Senior Country Officer, Telephone: (202) 473-0075

1. Pakistan is among the largest recipients of World Bank financial assistance. In FY2007, World Bank support to Pakistan totaled \$985 million, making it the seventh largest borrower in the world. The World Bank Group program in Pakistan consists of an integrated package of financial support, including IBRD lending, concessional IDA credits and IFC investments, along with complementary analytical and advisory services.

2. The Bank Group's Board of Directors endorsed a new Country Assistance Strategy (CAS) for Pakistan on June 1, 2006, covering fiscal years 2006 through 2009. The CAS outlines the Bank's strategic approach to helping Pakistan achieve its development goals over a four-year period. Despite recent good economic performance, Pakistan's development challenges remain formidable. The strategy addresses the key challenge of sustaining rapid growth in order to further reduce poverty. This will require accelerating human development so that the poor can participate in and benefit from growth. Sustained growth will also require sound macroeconomic management along with improvements in the investment climate, including regulatory reform and significant investment in infrastructure.

3. The CAS is designed around three main pillars:

- Sustained Growth and Improved Competitiveness: The principal focus of this pillar will be to support investments and reforms needed to improve the business environment for trade and investment and sustain rapid, private sector-led growth. The Bank will provide support to key sectors such as agriculture and infrastructure, and help the government strengthen macroeconomic management through improving public expenditures and supporting ongoing tax reforms.

- $\quad$ Strengthened Governance and Service Delivery: Priorities in this area will be to support further reforms and investment to increase efficiency, transparency, and accountability in the use of public resources, while supporting cross-cutting reforms needed to improve service delivery at all levels of government — with particular attention to health, education, water and sanitation, safety nets, and municipal services.

- Improved Lives and Protection of the Vulnerable: The Bank will focus on increased investment in the education and health sectors to help build the skilled, healthy work force needed to sustain strong growth. This area of the CAS also features targeted interventions to help the poor, including strengthened safety nets and targeted interventions and community-based approaches in rural areas. 
4. In line with Pakistan's recent performance and the government's request for increased Bank Group support, the CAS outlines a substantial increase in the volume of lending to Pakistan through FY2009. The expansion in lending will take place primarily in infrastructure (mainly in energy, water, and transport) and human development. One priority will be to continue to assist in addressing the impact of the October 2005 earthquake - up to $\$ 1$ billion will be used to support reconstruction and recovery. Overall, a flexible lending program of up to $\$ 6.5$ billion is set forth in the CAS. About half of this amount ( $\$ 3.1$ billion) will take the form of IDA credits. Policy-based lending will account for up to half of planned financial support.

5. The IFC strategy in Pakistan, as expressed in the CAS, seeks to increase investments with a target range of \$500-600 million during the FY2006-09 period. IFC activity will focus on three main sectors: financial, SME and infrastructure. IFC has also initiated a substantial TA program in Pakistan to build capacity and address constraints of the SME, infrastructure and financial sectors.

\section{IBRD/IDA financial operations since FY2002 are summarized below:}

Pakistan: World Bank Group Financial Operations

(In millions of U.S. dollars)

\begin{tabular}{|c|c|c|c|c|c|c|}
\hline & FY2002 & FY2003 & FY2004 & FY2005 & FY2006 & FY2007 \\
\hline \multicolumn{7}{|c|}{ Commitments } \\
\hline IBRD & $\ldots$ & $\ldots$ & 50 & 347 & 315 & 100 \\
\hline IDA & 800 & 297 & 731 & 500 & 1,161 & 885 \\
\hline \multicolumn{7}{|c|}{ Disbursements } \\
\hline IBRD & 38 & 40 & 13 & 203 & 149 & 154 \\
\hline IDA & 830 & 316 & 290 & 778 & 1,061 & 1,030 \\
\hline \multicolumn{7}{|c|}{ Repayments } \\
\hline IBRD & 255 & 257 & 277 & 299 & 289 & 265 \\
\hline IDA & 71 & 83 & 96 & 111 & 117 & 170 \\
\hline \multicolumn{7}{|c|}{ Debt Disbursed and Outstanding } \\
\hline IBRD & 2,820 & 2,706 & 2,526 & 2,406 & 2,247 & 2,132 \\
\hline IDA & 5,097 & 5,604 & 6,020 & 6,651 & 7,627 & 8,700 \\
\hline
\end{tabular}




\section{Statement by the IMF Staff Representative on Pakistan}

December 23, 2009

1. This statement provides information on developments since the staff report (EBS/09/207) was circulated to the Executive Board on December 15, 2009. These developments underscore the economic team's commitment to stabilization and reform. This information does not alter the thrust of the staff appraisal.

2. On December 16, the Supreme Court declared the 2007 National Reconciliation Ordinance (NRO) invalid. The NRO had halted a number of investigations, setting aside court cases and convictions against senior politicians and bureaucrats. This ruling allows these investigations and court cases to proceed.

3. The standstill on the debt of Dubai World has had a limited impact on the Pakistani economy and the banking system. Two Pakistani banks have exposure to the United Arab Emirates. One bank has Abu Dhabi exposure and is not affected by the standstill. The other bank, with exposure to Dubai World, has adequate capital to absorb any losses, according to the State Bank of Pakistan. Confidence in the banking system in Pakistan has been steady, with the interbank market working normally and deposits unaffected. The direct impact on trade and financial flows is likely to be small; Dubai accounts for 5-10 percent of these flows. If expatriate Pakistanis lose jobs in Dubai, remittances could be affected somewhat and the rapid growth experienced in July-November (29 percent) may not continue. After a limited initial impact, Pakistan's sovereign and CDS spreads have been little affected.

4. The National Finance Commission, responsible for revenue sharing arrangements between the federal and provincial governments, has concluded its work. Agreement was reached between the federal government and the provinces on revenue sharing for the next five fiscal years (2010/11-2015/16), which helps facilitate the introduction of a VAT. The commission decided to allot a greater share to the provinces (rising to 57.5 percent in 2011/12 compared to 47.5 percent currently). It also reallocated the distribution of revenue between provinces, with a larger share going to Baluchistan.

\section{The following policy commitments included in the Third Supplementary} Memorandum of Economic and Financial Polices have been implemented:

- $\quad$ Tax audits have commenced (a prior action). The Federal Board of Revenue (FBR) has identified 921 companies and associations for audit, across a wide range of sectors. On December 12, these audits started. To facilitate audits, the FBR has posted auditors to regional tax offices.

- $\quad$ Electricity reform has proceeded. The notification (implementation) of the quarterly tariff adjustment was done on December 21, 2009. 
- $\quad$ On December 21, the government submitted amendments to the State Bank of Pakistan (SBP) Act to parliament to increase the SBP's operational autonomy. Staff reviewed the draft amendments sent by the SBP to the government, but has not yet seen the final version of the amendments submitted to parliament.

6. On December 16, the federal cabinet approved a set of austerity measures. The government views these measures as providing a road map to achieve cost effective governance based on a model of rationalization of expenditure, better use of available resources, process re-engineering and efficiency of operation. The rationalization envisages a reduction in the number of federal ministries and government departments, and the devolution of responsibilities to the provinces. In addition, the plan envisages a restructuring of the eight public sector enterprises with a view to privatizing them. The government also plans to improve the budgetary process and overhaul the implementation of the public sector development program. Expenditure on utilities, furnishings, and use of vehicles will be standardized and reduced. The remuneration of government employees is under review with the objective of moving towards a pay for performance model with monetization of benefits. 
Press Release No. 09/480

International Monetary Fund

FOR IMMEDIATE RELEASE

Washington, D.C. 20431 USA

December 23, 2009

\section{IMF Completes Third Review Under Stand-By Arrangement for Pakistan, Approves US\$ 1.2 Billion Disbursement}

The Executive Board of the International Monetary Fund (IMF) today completed the third review of Pakistan's economic performance under a program supported by a Stand-By Arrangement (SBA). The completion of the review enables the immediate disbursement of an amount equivalent to SDR 766.7 million (about US\$1.2 billion), bringing total disbursements under the program to an amount equivalent to SDR 4.17 billion (about US\$6.54 billion).

The Executive Board also approved Pakistan's request for a waiver for the non-observance of the end-September performance criterion on the ceiling of the overall budget deficit, which was missed by a margin of 0.3 percent of GDP.

The 23-month SBA in an amount equivalent to SDR 5.17 billion (about US\$8.11 billion) was approved on November 24, 2008 (see Press Release No. 08/303). On August 7, 2009, the SBA was augmented to an amount equivalent to SDR 7.24 billion (about US\$11.35 billion) and extended to end 2010 (see Press Release No. 09/281).

Following the Executive Board's discussion on Pakistan, Mr. Murilo Portugal, Deputy Managing Director and Acting Chair, stated:

"The Pakistani authorities have made significant efforts to stay the course on stabilization and structural reform against the backdrop of weak external demand and a difficult security and political environment. Inflation has declined, the external position strengthened, and tangible progress has been made in tax, electricity, and financial sector reforms.

Nevertheless, Pakistan's vulnerabilities remain high, due notably to low revenue collection, large energy subsidies, and weak private sector credit. A credible fiscal consolidation, supported by flexible interest rate and exchange rate policies, further structural reforms, and improved governance will be essential to reduce these vulnerabilities. 
"The fiscal slippage in the first quarter of 2009/10 was due partly to factors beyond the authorities' control. The authorities are committed to strengthened fiscal discipline, including the use of contingencies. Adhering to the 2009/10 fiscal target—while challenging given security-related spending pressures - will help build confidence, preserve macroeconomic stability, and limit the potential for crowding out the private sector. Resolute implementation of tax administration reforms and timely disbursement of the pledged foreign financing will help facilitate fiscal management and prevent excessive recourse to domestic borrowing and undue compression of social and development spending.

"The introduction of the VAT and associated administrative reforms, scheduled for July 1, 2010 , is key to strengthening revenue, crucial for reducing poverty and financing needed investment in human and physical capital. Prompt submission of the VAT law to parliament and its passage will therefore be important.

"The nationwide rollout of the strengthened targeting system for the social safety net is key to easing the hardship related to macroeconomic adjustment. The authorities remain committed to strengthening administrative capacity to ensure timely implementation of the new system.

"A cautious monetary policy stance will be essential for counteracting inflationary pressures. Maintaining a flexible exchange rate policy will help facilitate a further buildup of foreign reserves. The early experience with the interest rate corridor is encouraging, but care should be taken to harmonize better the corridor-based liquidity management framework with the monetary policy objectives.

"The authorities remain committed to ensuring financial stability and fostering bank soundness. Continued vigilance will be needed to ensure that bank lending decisions are economically sound, and to avoid moral hazard risks related to regulatory forbearance. " 


\section{Statement by Mr. Mojarrad and Mr. Ahmad on Pakistan, Executive Director and Advisor for Pakistan December 23, 2009}

We would like to thank staff for their dedicated work and succinct report. We are especially grateful to management for assisting with countries that made pledges under the Friends of Pakistan meeting in Tokyo. We also wish to thank our friends, particularly those countries that have made good on their significant pledges in assisting Pakistan at this difficult juncture. The Fund has played an exemplary role in supporting an ambitious stabilization program, with the SBA providing an anchor for policy implementation and a catalytic role with donors.

The benefits of the stabilization program are evident, reserves are buoyant, core inflation has been brought down, and prudent management has led to a solvent financial sector. Stress tests indicate that Pakistani banks are well placed to sustain shocks. The interest rate corridor is working well, with the overnight repo rate kept within the requisite range. The Government's Pakistan Remittance Initiative has encouraged the channeling of remittances through official channels - and the anticipated decline in remittances has not materialized.

While there has been some improvement in growth, with an excellent wheat harvest, industry and trading remain depressed, and private sector demand for credit remains anemic.

Depressed activity levels have had an impact on imports (and associated revenues) as well as employment opportunities. Employment prospects have been further reduced by the sharp cuts in the planned development spending needed in order to meet the program's budget deficit target.

The fiscal targets were established at the outset of the program, before the extent of the global downturn was fully appreciated. It is clear that the revenue decline over the past year has been largely due to the exogenous reductions in activity level-more than offsetting the increase in the standard rate of GST during 2008/9. To some extent, the restructuring in the Federal Board of Revenue since 2009 has also contributed to the revenue decline, especially given the legal challenges to the creation of an Inland Revenue Service since March 2009. However, the contraction in revenue bases continued during the first quarter, and despite the imposition of the Petroleum Levy since July 2009 and drastic reductions in public investment, the fiscal deficit target for end September was narrowly missed. The slippages have been largely made up since then, mainly due to the tight controls over investment spending. The government intends to meet the budget target for the year. The authorities have agreed with staff to invoke a second-best measure, should it prove necessary, involving an increase in the special excise surcharges that effectively raise tariffs.

Despite the difficult security situation - in fact Pakistan is virtually a war economy - there has been significant progress on the structural reform front. With a resurgent Parliament, 
provinces run by different parties, and an active judiciary, there has to be a full and open discussion of government policies. A recent attempt by an international agency to bypass parliament led to a severe reaction, and any additional attempts to restrict the Parliament are likely to be counterproductive. Recent actions of the Supreme Court also lay the basis for greater transparency and accountability in the future.

Yet the structural reform agenda remains on track, and benefits from broad-based support. This is seen most dramatically in the recent award of the National Finance Commission, during which provinces agreed that the Federation should administer the reformed VAT, but the revenues attributed to services (assigned to provinces under the Constitution) be returned to them in full. It should be noted that this is the first NFC award in twelve years, and that even General Musharraf at the height of his powers was unable to agree an award with the provinces.

Pakistan has had a VAT since 1990 - except that it was called a GST and did not work very well. As we have mentioned in earlier statements to the Board, a World Bank tax reform project was initiated in the early 2000s. Unfortunately, the base was gradually eroded as the weak administration was unable to cope with refunds. The "temporary" policy response led to widespread domestic zero-rating for the main productive sectors. The situation was exacerbated by a decision of the authorities in 2004 to dispense with the auditing function, which was effectively suspended.

The resurrection of the tax administration reforms in 2008, supported by a Bank team lead by a former FAD staff member, has led to the integration of functions - this was at the heart of the tax reform project initiated in 2000. The creation of the Inland Revenue Service in 2009 was challenged in the High Courts, but the Government has prevailed. The tax administration reforms are making steady progress commensurate with building an effective support for the new organization. The audit process has been resurrected with some fanfare-with prominent firms being chosen in a random audit process conducted in full public view last week-with enhanced transparency to illustrate that no political vendettas are involved in the audit process.

There was a need to clean up and modernize the legal framework for the GST, also to reflect the advances made to extend coverage agreed under the recent NFC award. This is why a new law has been drafted, rather than a simple cleaning up of the old law. The name has been changed also to reflect the "psychological break" with the past. A new draft law for the taxation of goods and services has been prepared with the assistance of international experts, including from the Fund and the Bank, and is being discussed with key stakeholders. Although it would have been preferable to allow for more time for discussion with the interested parties, the government intends to submit the law to Parliament by end-December as agreed under the program. However, binding Parliament to a specific date for passage of the new law may be counterproductive. 
The heart of the reform of the taxation of goods and services lies in an expansion of the base. This will be facilitated with an automated VAT refunds mechanism that has been initiated, but it is essential that this new facility be fully tested before full rollout to ensure that it does not facilitate large-scale leakages. The strengthening of the audit capability is key, and the experience developed in the coming months will help. The government's intention remains to implement a broad-based tax on goods and services by the start of the next fiscal year.

With respect to the Treasury Single Account (TSA), as stated before, the government had ensured with the establishment of zero-balance assignment accounts that there would be no accretion to the balances in accounts held outside the Federal Consolidated Fund/TSA. The issue of the stock of balances held in commercial was addressed in a Bank Technical Assistance mission in September, 2009. Subsequently, a survey of existing balances was conducted and it was determined that most of these were due to legal requirements imposed by donors, including the World Bank, or were not strictly classifiable as government funds (e.g., autonomous income of universities through fees and other services). The remaining balances that should be in the TSA will be transferred as agreed by the end of the fiscal year, keeping in view the impact on the banking system.

The budget, as well as the Fund supported program, remains predicated on disbursements of funds from the Friends of Pakistan, promised in Tokyo. Continued success under the program, at this very difficult juncture, depends on these resources materializing within the envisaged timeframe, and we very much hope that our friends will keep to their commitments. 